\title{
Constraint Programming-Based Transformation Approach for a Multi-Objective \& Multi-Mode Resource Investment Project Scheduling Problem Under Mixed Fuzzy-Stochastic Uncertainties
}

Kemal Subulan ( $\nabla$ kemal.subulan@gmail.com )

Dokuz Eylul University: Dokuz Eylul Universitesi https://orcid.org/0000-0001-7640-1976

Gizem Çakır

Dokuz Eylul University: Dokuz Eylul Universitesi

\section{Research Article}

Keywords: Resource investment project scheduling problem, constraint programming, fuzzy mathematical programs, scenario-based stochastic programs, ERP implementation projects

Posted Date: July 7th, 2021

DOl: https://doi.org/10.21203/rs.3.rs-613012/v1

License: (c) (1) This work is licensed under a Creative Commons Attribution 4.0 International License. Read Full License 
Constraint programming-based transformation approach for a multi-objective \& multimode resource investment project scheduling problem under mixed fuzzy-stochastic uncertainties

\author{
Kemal Subulan ${ }^{* 1}$, Gizem Çakır² \\ ${ }^{I}$ Dokuz Eylül University, Faculty of Engineering, Department of Industrial Engineering, Izmir, Turkey \\ ${ }^{2}$ Dokuz Eylül University, The Graduate School of Natural and Applied Sciences, Buca, Izmir, Turkey,
}

\begin{abstract}
In fuzzy mathematical programming literature, most of the transformation approaches were mainly focused on integer linear programs (ILPs) with fuzzy parameters/variables. However, these ILP-based solution approaches may be inadequate for solving large-scaled combinatorial fuzzy optimization problems, like project scheduling under fuzzy-stochastic environments. Moreover, many project scheduling applications may contain different types of uncertainties such as fuzziness, stochasticity, dynamism etc. simultaneously in real-life settings. Based on these motivations, this paper presents a novel constraint programming (CP) based transformation approach for solving a multi-objective and multi-mode fuzzy-stochastic resource investment project scheduling problem (FS-MRIPSP) which is a well-known NPcomplete problem. In fact, the proposed solution approach mainly depends on a bound \& decomposition principle which divides fuzzy components of the problem into crisp middle, lower and upper level problems. Thus, it reduces the problem dimension and does not need to use any standard fuzzy arithmetic and ranking operations directly. Furthermore, stochastic nature of the problem is also taken into account by using a multi-scenario based stochastic programming technique. Finally, a weighted additive fuzzy goal program (WAFGP) is embedded into the proposed CP-based transformation approach in order to produce compromise fuzzy project schedules which trade-off between the expected values of project makespan and total resource usage costs. To show validity and practicality of the proposed approach, a real-life application is also presented for a production-and-operations management (POM) module implementation process of an international Enterprise Resource Planning (ERP) software. The generated fuzzy project schedules under different scenarios by the proposed CPbased approach are also compared to the results of a similar ILP-based transformation approach. Computational results have shown that the proposed $\mathrm{CP}$-based approach outperforms than the ILP-based approach in terms of both solution quality and computational time.
\end{abstract}

Keywords: Resource investment project scheduling problem; constraint programming; fuzzy mathematical programs; scenario-based stochastic programs; ERP implementation projects.

\title{
1. Introduction
}

Most of the classical transformation approaches in fuzzy mathematical programming literature utilized standard fuzzy arithmetic operations or Zadeh's extension principle with fuzzy ranking techniques while converting a fuzzy optimization problem into its crisp equivalent form. However, some recent studies (Subulan, 2020; Sotoudeh-Anvari, 2020; Baykasoğlu \& Subulan, 2017, 2019) have proven that standard fuzzy arithmetic-based transformation approaches may cause questionable results or unrealizable solutions for many real-life engineering applications. For instance, several risky situations may arise in project scheduling and transportation applications under fully fuzzy environments (Baykasoğlu \& Subulan; 2017; 2019; Subulan, 2020). Additionally, these standard fuzzy arithmetic-based transformation approaches are mainly developed depending upon integer linear programs (ILPs) with fuzzy parameters and variables. On the other hand, ILP-based solution approaches are not able to handle large-sized combinatorial fuzzy optimization problems like resource constrained project scheduling problem (RCPSP) and its variant, i.e., resource investment 
project scheduling problem (RIPSP). Fortunately, many studies available in the literature have demonstrated that CP-based solution methodologies are able to produce promising results for deterministic project scheduling problems (Liess \& Michelon, 2008; Berthold et al., 2010; Li \& Womer 2015; Hauder et al., 2020; Kong \& Dou, 2021). On the other hand, none of them handled such a fully uncertain project scheduling problem under a mixed fuzzy and stochastic environment. It should be mentioned here that the problem size and therefore, model dimension including the number of constraints and decision variables increase significantly after realizing a transformation from fuzzy-stochastic version to crisp equivalent form. For that reason, it may be useful to develop a CP-based solution approach in order to convert the fuzzy-stochastic model into its crisp equivalent form. To the best of our knowledge, there is no available study in the literature on a CP-based transformation approach for solving fuzzy or fuzzy-stochastic mathematical programming problems. Actually, this is the main contribution of this research to the fuzzy mathematical programming literature.

As mentioned before, we discussed a multi-objective and multi-mode RIPSP with mixed fuzzy and stochastic project parameters/variables throughout this paper. Apart from the classical RCPSPs, resources' capacities or availability levels are defined as decision variables instead of project parameters or input data in RIPSPs or resource availability cost problem (RACP). Moreover, many real-life project scheduling cases involve different types of uncertainties such as fuzziness, randomness, and dynamism etc. concurrently. However, there is still a lack of studies in the literature on fuzzy-stochastic and/or fuzzy-random project scheduling problems which are composed of mixed fuzzy and stochastic project parameters/variables. Based on these motivations, this paper introduces a novel CP-based transformation approach with a bound \& decomposition principle. In fact, some project parameters such as the usage costs of renewable resources, activity durations and therefore, their earliest/latest finishing times are taken as uncertain data and represented by triangular fuzzy numbers. By the way, stochastic nature of some project parameters, i.e., resource requirements of the renewable/non-renewable resources are also taken into account by a multiscenario based stochastic programming approach. In addition to these fuzzy and stochastic project parameters, completion times of the project activities are also defined as mixed fuzzystochastic decision variables because of the fuzzy activity durations. Furthermore, availability levels of both renewable and non-renewable resources are also stated as mixed fuzzy-stochastic variables which represent fuzzy resource capacities under different scenarios. Therefore, the proposed transformation approach is also able to generate fuzzy project schedules and resource availabilities under different scenarios. Briefly, the core contributions of this research can be summarized as follows. (i) This paper introduces a fully uncertain, multi-objective and multimode RIPSP with fuzzy, stochastic parameters and fuzzy-stochastic decision variables as well. In fact, all of the project input data and outputs are stated as uncertain and several fuzzy project schedules with fuzzy availabilities of the renewable/non-renewable resources are generated under different scenarios. (ii) A new CP-based transformation approach with a bound \& decomposition principle is developed to solve a realistic size problem instance under a reasonable computing time. In multi-objective optimization phase, different search strategies are also tested to obtain better quality solutions within shorter run times. Computational results of the proposed CP-based solution approach are also compared to the results of a similar ILPbased method. It was proven that CP-based transformation approaches may provide superior results in terms of both solution quality and computational time under mixed fuzzy-stochastic environments.

The rest part of this paper is organized as follows: A brief literature review on fuzzystochastic project scheduling problems is presented in Section 2. Problem description and its mathematical programming formulations are given in Section 3. In Section 4, fundamentals of the proposed CP and ILP-based transformation approaches with a bound \& decomposition 
principle are described. Then, the proposed approaches are illustrated based on a trivial numerical example of a classical MRCPSP under fuzzy-stochastic environments. In the same section, a WAFGP is also embedded in the proposed ILP and CP-based approaches so as to handle multi-objective nature of the FS-MRIPSP. The validity and practicality of the proposed $\mathrm{CP}$-based transformation approach is also tested on a real-life ERP implementation project data in Section 5. Comparison of the computational results is also carried out in that section. Finally, conclusions and future research directions are discussed in Section 6.

\section{A literature review on fuzzy-stochastic project scheduling problems}

In this section, a literature survey on fuzzy-stochastic and fuzzy-random project scheduling problems is presented. In fact, researches in this field can be mainly classified into three parts, i.e., fuzzy, stochastic and fuzzy-random project scheduling problems according to the type of uncertainties (Subulan 2019; Subulan, 2020). Most of the previous researches handled the uncertain project parameters/input data by making use of probability or fuzzy set theories. Specifically, they generally considered the uncertain project parameters as stochastic input data which were defined as random variables with known probability distributions and proposed several stochastic programming based solution techniques (Malcolm et al., 1959; Jørgensen, 1999; Ashtiani et al., 2011; Artigues et al., 2013; Li \& Womer, 2015; Wang et al., 2015; Tseng \& Ko, 2016; Chand et al., 2016; Chakrabortty et al., 2017; Tao et al., 2018; Uysal et al., 2018; Rostami et al., 2018; Bruni et al., 2018; Chen et al., 2018; Chen, et al., 2019; Rezaei et al., 2020; Sallam et al., 2021; Balouka \& Cohen, 2021; Chen et al., 2021). On the other hand, some of the other remaining researches defined the uncertain project data as fuzzy parameters and developed fuzzy mathematical programming based solution approaches (Hapke et al., 1994; Hapke \& Slowinski, 1996; Ozdamar \& Alanya, 2001; Pan et al., 2001; Wang, 2002; Wang, 2004; Liu et al., 2007; Long \& Ohsato, 2008; Bhaskar et al., 2011; Atli \& Kahraman, 2012; Masmoudi \& Hait, 2013; Huang et al., 2013; Atli \& Kahraman, 2014; Knyazeva et al., 2015; Kaveh et al., 2016; Sajadi et al., 2017; Khalilzadeh et al., 2017; Yousefli, 2017; Zhang et al., 2018; Roghanian et al., 2018; Birjandi \& Mousavi, 2019; Mahdavi et al., 2021). In fact, there are lots of studies either on stochastic or fuzzy project scheduling problems. In other words, many existing researches have focused on only one type of uncertainty, either fuzziness or stochasticity. Specifically, different uncertainty types were usually handled separately by most of the former preliminary studies. However, many real-life project scheduling applications may contain different types of uncertainties concurrently. Actually, some project parameters may contain only fuzziness or randomness whereas others may involve them jointly. Therefore, different types of uncertainties in project parameters/variables should be modelled and handled in an integrated way. As highlighted by Alipouri et al. (2020) that some project activities have been formerly realized in several times, and therefore, there are sufficient historical data related to their durations, resource requirements etc. Nevertheless, other project activities may rarely or never been performed before and therefore, they may have no historical data. In this case, randomness and fuzziness arise instantaneously and the corresponding project scheduling problem can be named as fuzzy-stochastic. Unfortunately, there is still a lack of studies in the literature on fuzzy-stochastic or fuzzy-random project scheduling problems. Actually, its literature is in its burn-in phase. An overview of the recent articles which focused on modelling and solving hybrid fuzzy-random project scheduling problems can be summarized as follows: The fuzzy-stochastic project scheduling problem has been first initiated by Ke and Liu (2007) in the literature. They designed a hybrid intelligent algorithm based on GA for solving a project scheduling problem with mixed uncertainty of randomness and fuzziness. They supposed that activity durations will be random fuzzy variables and then, three different types of random fuzzy models, i.e., expected cost minimization, $(\alpha, \beta)$-cost minimization and chance maximization models were proposed to satisfy several management needs. A similar project 
scheduling problem was also presented by Ke et al. (2012) in which the philosophy of fuzzy random programming was introduced depending upon different ranking criteria of fuzzy random variables. Ke and Ma (2014) also modelled the project time-cost trade-off problem with fuzzy random activity durations. Similar to their previous researches, they developed three types of models and designed an intelligent algorithm which combines fuzzy random simulations with GA to solve them. Huang et al. (2009) developed a hybrid intelligent algorithm based on GA and random fuzzy variables for solving an expected cost model of a software project scheduling problem with random fuzzy activity duration times. A linearized MIP technique was developed by Nematian et al. (2010) based on the expected value of fuzzyrandom variables, fuzzy inequality approaches and max-min operator of Bellman \& Zadeh (1970) for solving a RCPSP with fuzzy-random activity durations, ready times and project deadline. A similar MIP-based transformation approach was also employed by Artykov and Atymtayeva (2015) by focusing on fuzzy-random activity durations only. Xu and Zhang (2012) proposed a hybrid GA with a fuzzy logic controller to solve the multi-objective resourceconstrained multiple project scheduling problem with fuzzy-random resource consumptions and due-dates. A new solution approach which hybridizes adaptive particle swarm optimization (PSO) with GA and a fuzzy-random simulation algorithm was developed by Gang et al. (2013) for a multi-project RCPSP including fuzzy-random activity durations and resource costs. Zhang (2014) designed a bi-level multi-objective PSO algorithm for solving a RCPSP with fuzzyrandom activity durations, resource availabilities and tardiness penalty cost values. In a similar way, a combinatorial-priority-based hybrid PSO algorithm was presented by $\mathrm{Xu}$ and Feng (2014) for a multi-mode resource-constrained multiple project scheduling problem. The normal/crashed durations, resource consumptions, variable and crashing costs were also represented by fuzzy-random variables. Xu et al. (2015) developed a fuzzy random simulationbased bi-level global-local-neighbor PSO algorithm for a multiple decision maker project scheduling problem in a fuzzy-random environment. They aimed at maximizing the profit, minimizing the project makespan and project costs which correspond to the objectives of different project stakeholders. In order to formulate a robust optimization model of a preemption RCPSP with fuzzy-random activity durations and resource consumptions, a proactive strategy was proposed by Chen and Zhang (2016). Unlike the previously mentioned researches, they also took into account the activity splitting concept. A bi-level fuzzy random multiple objective model is developed by Zhang et al. (2019) for solving a multi-mode RCPSP in which costs, revenues and resource requirements of the project activities were defined as fuzzy-random variables. A self-adaptive differential evolution hyper-heuristic algorithm was developed by Alipouri et al. (2019) to minimize the expected makespan of a FS-RCPSP in which the activity durations were described as fuzzy-random variables. They recommended that modelling other project parameters, i.e., the resource requirements of the activities as fuzzy-random variables may find a research merit in the future studies. Alipouri et al. (2020) also presented a resource flow network based mathematical model with fuzzy random variables to solve a fuzzy-stochastic RCPSP. They converted that original model to an LP with fuzzy variables/parameters by using the expected values of fuzzy-random variables. Afterwards, this model was also transformed into an MILP model with final crisp parameters/variables. To test performance of that model, several benchmark instances were also generated and solved by an optimization software. A hybrid interval programming and chance constrained optimization based approach was developed by Subulan (2020) for solving a multi-objective and multi-mode RIPSP under mixed interval and stochastic uncertainties. This approach is also able to produce more reliable and risk-free compromise project schedules according to the project manager's risk attitude. 
Table 1 Summary of the literature review on project scheduling problems under mixed fuzzy and stochastic uncertainties

\begin{tabular}{|c|c|c|c|c|c|c|c|c|c|c|c|c|c|c|}
\hline \multirow[t]{2}{*}{ Article } & \multirow[t]{2}{*}{ Uncertainty } & \multirow{2}{*}{$\begin{array}{c}\text { Problem } \\
\text { type }\end{array}$} & \multirow{2}{*}{$\begin{array}{c}\text { Multi- } \\
\text { objective }\end{array}$} & \multirow{2}{*}{$\begin{array}{l}\text { Multi- } \\
\text { mode }\end{array}$} & \multirow{2}{*}{$\begin{array}{l}\text { Multiple } \\
\text { projects }\end{array}$} & \multirow{2}{*}{$\begin{array}{c}\text { Risk } \\
\text { attitude }\end{array}$} & \multicolumn{6}{|c|}{ Uncertain components } & \multirow{2}{*}{$\begin{array}{l}\text { Application } \\
\text { or case study }\end{array}$} & \multirow[t]{2}{*}{ Solution methodology } \\
\hline & & & & & & & Durations & $\begin{array}{l}\text { Resource } \\
\text { req. }\end{array}$ & $\begin{array}{c}\text { Renewable res. } \\
\text { availability }\end{array}$ & $\begin{array}{l}\text { Non-renewable } \\
\text { res. availability }\end{array}$ & $\begin{array}{l}\text { Objective values } \\
\text { (Makespan etc.) }\end{array}$ & $\begin{array}{c}\text { Project } \\
\text { schedules }\end{array}$ & & \\
\hline Ke \& Liu (2007) & $\begin{array}{l}\text { Random } \\
\text { fuzzy }\end{array}$ & PSP & & & & & $\checkmark$ & & & & & & N.A & $\begin{array}{l}\text { GA-based hybrid } \\
\text { intelligent algorithm }\end{array}$ \\
\hline Ke et al. (2012) & $\begin{array}{l}\text { Fuzzy } \\
\text { random }\end{array}$ & PSP & & & & & $\checkmark$ & & & & & & N.A & $\begin{array}{l}\text { Fuzzy random } \\
\text { simulations with GA }\end{array}$ \\
\hline $\mathrm{Ke} \& \mathrm{Ma}(2014)$ & $\begin{array}{l}\text { Fuzzy } \\
\text { random }\end{array}$ & TCTP & & & & & $\checkmark$ & & & & & & N.A & $\begin{array}{l}\text { Fuzzy random } \\
\text { simulations with GA }\end{array}$ \\
\hline Huang et al. (2009) & $\begin{array}{l}\text { Random } \\
\text { fuzzy }\end{array}$ & PSP & & & & & $\checkmark$ & & & & & & $\begin{array}{c}\text { Software } \\
\text { development }\end{array}$ & $\begin{array}{l}\text { GA-based hybrid } \\
\text { intelligent algorithm }\end{array}$ \\
\hline $\begin{array}{c}\text { Nematian et al. } \\
\text { (2010) }\end{array}$ & $\begin{array}{l}\text { Fuzzy } \\
\text { random }\end{array}$ & RCPSP & & & & & $\checkmark$ & & & & $\checkmark$ & $\checkmark$ & N.A & $\begin{array}{l}\text { MILP-based } \\
\text { transformation method }\end{array}$ \\
\hline $\begin{array}{c}\text { Artykov \& } \\
\text { Atymtayeva (2015) }\end{array}$ & $\begin{array}{l}\text { Fuzzy } \\
\text { random }\end{array}$ & RCPSP & & & & & $\checkmark$ & & & & $\checkmark$ & $\checkmark$ & N.A & $\begin{array}{l}\text { MILP-based } \\
\text { transformation method }\end{array}$ \\
\hline Xu \& Zhang (2012) & $\begin{array}{l}\text { Fuzzy } \\
\text { random }\end{array}$ & rc-mPSP & $\checkmark$ & & $\checkmark$ & & & $\checkmark$ & & & & & $\begin{array}{l}\text { Water conservancy } \\
\& \text { hydropower } \\
\text { construction }\end{array}$ & $\begin{array}{l}\text { Hybrid GA with fuzzy } \\
\text { logic controller }\end{array}$ \\
\hline Gang et a1. (2013) & $\begin{array}{l}\text { Fuzzy } \\
\text { random }\end{array}$ & RCPSP & & $\checkmark$ & $\checkmark$ & & $\checkmark$ & & & & & & $\begin{array}{l}\text { Industrial } \\
\text { Equipment } \\
\text { Installation }\end{array}$ & $\begin{array}{c}\text { Fuzzy random } \\
\text { simulation-based hybrid } \\
\text { PSO \& GA }\end{array}$ \\
\hline Zhang (2014) & $\begin{array}{l}\text { Fuzzy } \\
\text { random }\end{array}$ & RCPSP & $\checkmark$ & & & & $\checkmark$ & $\checkmark$ & $\checkmark$ & & & & N.A & $\begin{array}{l}\text { Bi-level multiple } \\
\text { objective PSO } \\
\text { algorithm }\end{array}$ \\
\hline Xu \& Feng (2014) & $\begin{array}{l}\text { Fuzzy } \\
\text { random }\end{array}$ & RCPSP & $\checkmark$ & $\checkmark$ & $\checkmark$ & & $\checkmark$ & $\checkmark$ & & & & & $\begin{array}{l}\text { Hydropower } \\
\text { Construction }\end{array}$ & $\begin{array}{c}\text { Combinatorial priority- } \\
\text { based hybrid PSO }\end{array}$ \\
\hline Xu et al. (2015) & $\begin{array}{l}\text { Fuzzy } \\
\text { random }\end{array}$ & PSP & $\checkmark$ & $\checkmark$ & & & $\checkmark$ & & & & & & $\begin{array}{l}\text { Hydropower station } \\
\text { construction }\end{array}$ & $\begin{array}{c}\text { Fuzzy random } \\
\text { simulation-based PSO }\end{array}$ \\
\hline $\begin{array}{c}\text { Chen \& Zhang } \\
(2016)\end{array}$ & $\begin{array}{l}\text { Fuzzy } \\
\text { random }\end{array}$ & PRCPSP & & & & & $\checkmark$ & $\checkmark$ & & & & & N.A & $\begin{array}{l}\text { A transformation method } \\
\text { with a proactive strategy }\end{array}$ \\
\hline Zhang et al. (2019) & $\begin{array}{l}\text { Fuzzy } \\
\text { random }\end{array}$ & RCPSP & $\checkmark$ & $\checkmark$ & & & & $\checkmark$ & & & & & $\begin{array}{l}\text { Photovoltaic power } \\
\text { generation plant }\end{array}$ & $\begin{array}{c}\text { Multi-objective adaptive } \\
\text { PSO }\end{array}$ \\
\hline $\begin{array}{l}\text { Alipouri et al. } \\
\text { (2019) }\end{array}$ & $\begin{array}{l}\text { Fuzzy } \\
\text { stochastic }\end{array}$ & RCPSP & & & & & $\checkmark$ & & & & $\checkmark$ & $\checkmark$ & N.A & $\begin{array}{l}\text { Self-adaptive DE hyper- } \\
\text { heuristic }\end{array}$ \\
\hline $\begin{array}{l}\text { Alipouri et al. } \\
(2020)\end{array}$ & $\begin{array}{l}\text { Fuzzy } \\
\text { stochastic }\end{array}$ & RCPSP & & & & & $\checkmark$ & & & & $\checkmark$ & $\checkmark$ & N.A & $\begin{array}{l}\text { MILP-based } \\
\text { transformation method }\end{array}$ \\
\hline Subulan (2020) & $\begin{array}{l}\text { Interval- } \\
\text { stochastic }\end{array}$ & RIPSP & $\checkmark$ & $\checkmark$ & & $\checkmark$ & $\checkmark$ & $\checkmark$ & $\checkmark$ & $\checkmark$ & $\checkmark$ & $\checkmark$ & $\begin{array}{l}\text { ERP software } \\
\text { implementation }\end{array}$ & $\begin{array}{c}\text { Hybrid interval \& } \\
\text { chance-constrained } \\
\text { programs }\end{array}$ \\
\hline The present paper & $\begin{array}{l}\text { Fuzzy- } \\
\text { stochastic }\end{array}$ & RIPSP & $\checkmark$ & $\checkmark$ & & & $\checkmark$ & $\checkmark$ & $\checkmark$ & $\checkmark$ & $\checkmark$ & $\checkmark$ & $\begin{array}{l}\text { ERP software } \\
\text { implementation }\end{array}$ & $\begin{array}{l}\text { Constraint programming } \\
\text { (CP) }\end{array}$ \\
\hline
\end{tabular}

N.A - Not Available; PSP - Project Scheduling Problem; TCTP - Project Time-Cost Trade-off Problem; RCPSP - Resource-Constrained Project Scheduling Problem

rc-mPSP - Resource-Constrained Multiple Project Scheduling Problem; PRCPSP - Preemption RCPSP; RIPSP - Resource Investment Project Scheduling Problem 
Lastly, all of the previously discussed studies on fuzzy-stochastic project scheduling problems can be summarized and categorized as in Table 1 according to the problem type, problem characteristics (multi-objectivity, multiple execution modes, multiple projects, risk considerations etc.), uncertain parameters/variables, application study and the solution method. It was recognized in Table 1 that most of these existing studies have concentrated on classical RCPSPs under fuzzy-stochastic or fuzzy-random environments. Actually, there is a limited number of researches on fuzzy, stochastic and fuzzy-stochastic RIPSPs or RACPs in the literature (Yamashita et al., 2007; Xiong et al., 2011; Xiong et al., 2014; Subulan, 2020). In other words, RIPSPs or RACPs were commonly handled under deterministic environments (Drexl \& Kimms, 2001; Hsu \& Kim, 2005; Najafi \& Niaki, 2006; Shadrokh \& Kianfar, 2007; Ranjbar et al., 2008; Najafi \& Azimi, 2009; Javanmard et al., 2017; Zhu et al., 2017; Merrikhi et al., 2018; Jedrzejowicz \& Ratajczak-Ropel, 2019). Additionally, resource requirements of the project activities and availability levels or capacities of the renewable and non-renewable resources were generally taken as deterministic parameters in most of the available studies. Moreover, project schedules or starting/completion times of the activities which correspond to the decision variables took deterministic values in most of the previously mentioned studies. However, project schedules should also be defined as uncertain variables in such fully uncertain environments where all of the project parameters are stated as fuzzy, stochastic or fuzzystochastic data (Subulan, 2019; 2020; Baykasoğlu \& Subulan, 2017; 2019).

\section{Problem description and mathematical formulation of FS-MRIPSP}

This section presents a multi-objective FS-MRIPSP which is reformulated based on the discrete-time binary integer programming model of the classical RCPSP by Pritsker et al. (1969). In fact, that preliminary model is extended in this research by incorporating mixed fuzzy and stochastic types of uncertainties into a multi-objective and multi-mode RIPSP. Recently, Subulan (2020) has already proposed another variant of that original model by considering mixed stochastic and interval types of uncertainties. In fact, a hybrid chance-constrained optimization and interval programming based solution approach was developed in order to handle mixed interval and stochastic types of uncertainties simultaneously. It was also highlighted by Subulan (2020) that fuzzy version of the examined problem can also be studied in the future since epistemic uncertainty of some project parameters/data can be better reflected by using fuzzy set theory. Because, fuzzy numbers may provide more information on the uncertain project data instead of using interval/grey numbers. Because, interval numbers just take into account the lower and upper bounds on the imprecise project parameters. Thus, the membership function values of several possible outcomes are ignored unfortunately. For that reason, some of the project parameters which have epistemic uncertainties are represented by triangular fuzzy numbers instead of interval valued numbers in this paper. Moreover, from the theoretical and methodological perspectives, instead of using a chance-constrained stochastic optimization method, this paper employs a multi-scenario based stochastic programming approach to cope with the random project parameters and avoid the risk of constraint violation. In detail, renewable/non-renewable resource requirements of the project activities are assumed to be stochastic parameters since they may have randomness in nature. Therefore, values of these random project parameters are represented by discrete scenarios with the probability of each outcome. On the other hand, availability levels or capacities of both renewable \& nonrenewable resources are defined as fuzzy-stochastic decision variables and therefore, it is targeted to determine their ideal fuzzy values under each scenario. Because of these structural modifications, the examined problem namely FS-MRIPSP will have a multi-objective nature since effective and efficient resource utilizations may be as important as traditional project makespan objective. Due to the ambiguity/vagueness in the activity durations, their earliest/latest finishing times can be seen as the other critical issue when modelling and solving 
real-life MRIPSPs under uncertainty. Actually, project managers/experts may not know certain values of these project parameters in advance, but they may easily specify their pessimistic, most likely and optimistic values according to their knowledge and expertise. For that reason, instead of using probability theory, fuzzy sets and possibility theory can be utilized to handle these uncertain project parameters. In summary, characteristics/features of the FS-MRIPSPS can be outlined as in Fig. 1.

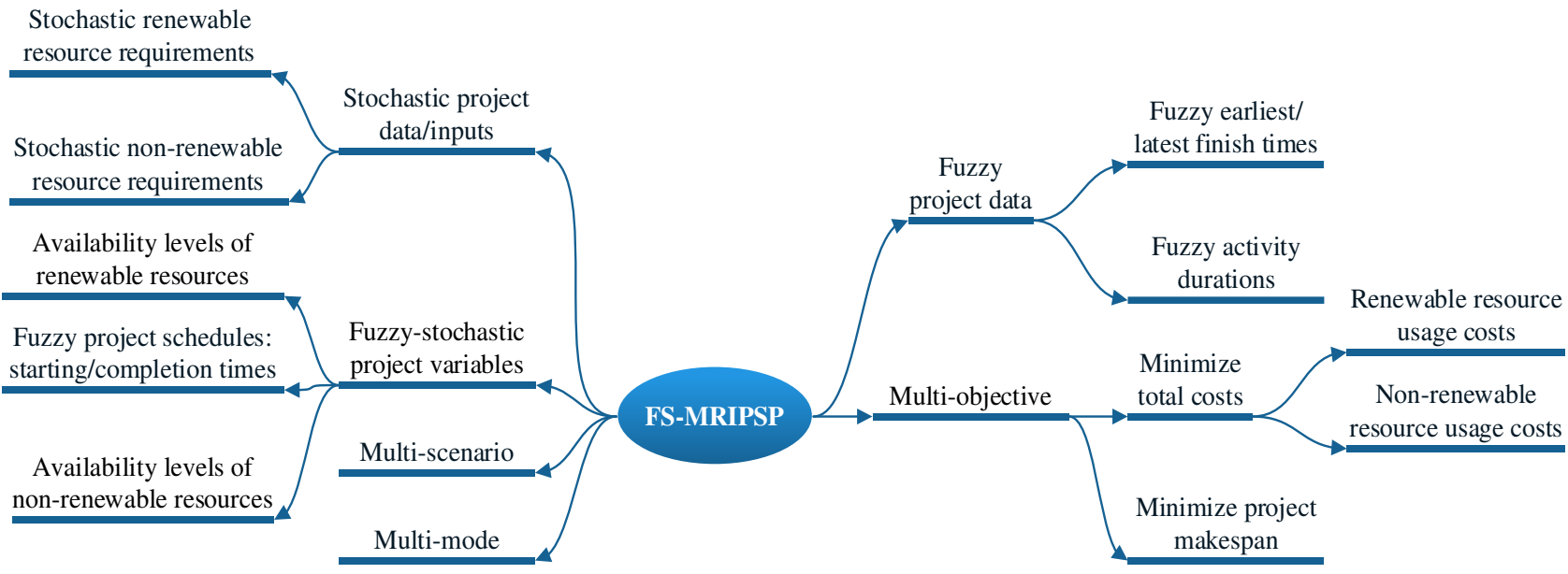

Fig. 1 Summary of characteristics/features of the FS-MRIPSP

\subsection{Integer linear programming (ILP) model formulation of the multi-objective FS-MRIPSP}

In light of these facts, ILP model formulation of the FS-MRIPSP is first presented in Eqs. (1) - (8) by using the mathematical nomenclature in Table 2. Actually, the following formulation corresponds to a scenario-based stochastic \& possibilistic programming model of the multi-objective FS-MRIPSP.

Table 2 Mathematical nomenclature for the integer programming (IP) formulation of the FS-MRIPSP

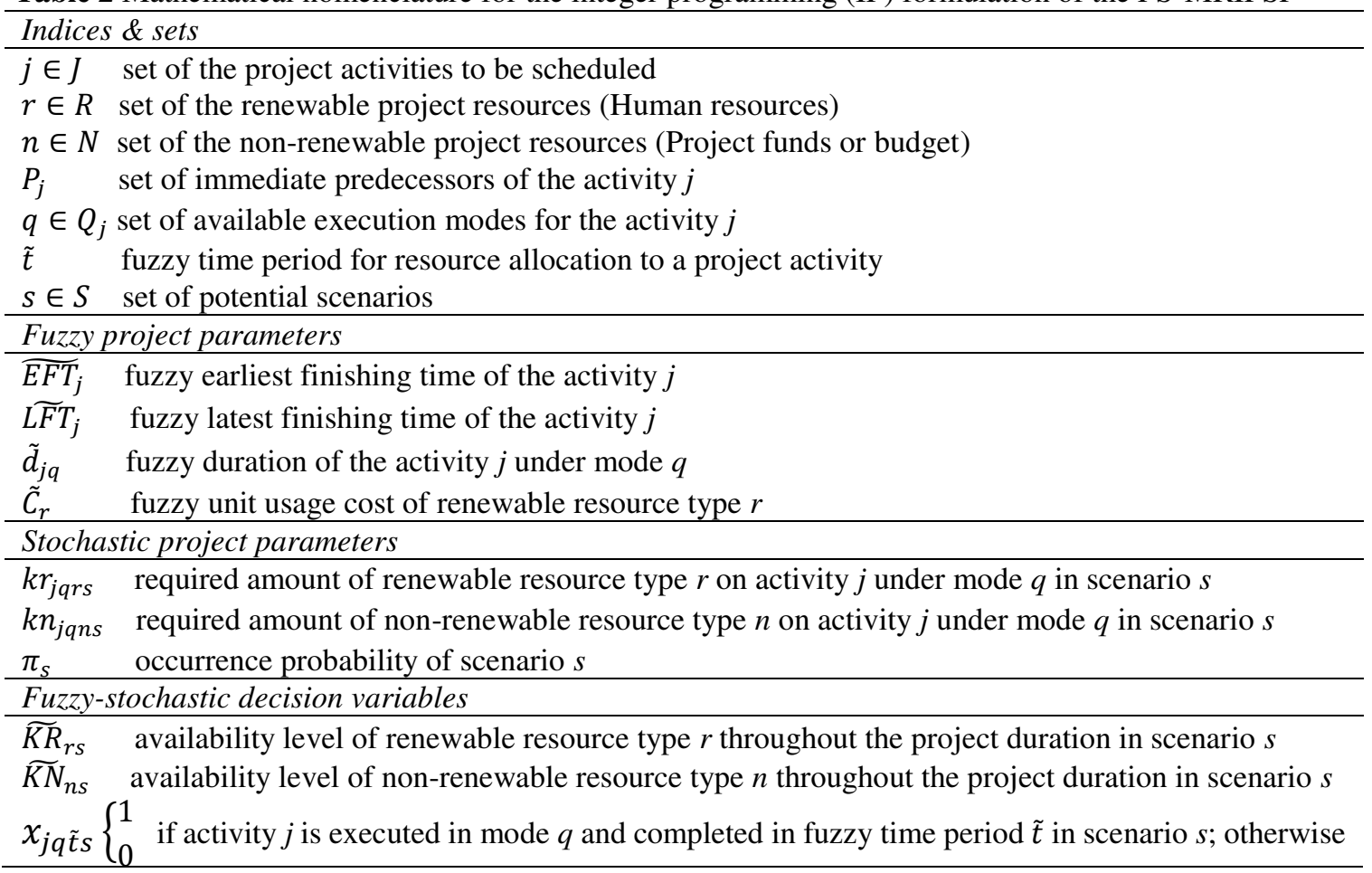


Minimize EV(Project Makespan) $\tilde{Z}_{1} \cong \sum_{\tilde{t} \in \widetilde{E F T_{J}}}^{L \widetilde{F T} T_{J}} \sum_{S=1}^{S} \pi_{S} \cdot \tilde{t} \cdot x_{J 1 \tilde{t} s}$

Minimize EV(Total Resource Costs) $\tilde{Z}_{2} \cong \sum_{n=1}^{N} \sum_{s=1}^{S} \pi_{s} \cdot \widetilde{K N}_{n s}+\sum_{r=1}^{R} \sum_{s=1}^{S} \pi_{s} \cdot \widetilde{K R}_{r s} \cdot \tilde{C}_{r}$

The model constraints of the FS-MRIPSP are formulated as in the following Eqs. (3) - (8).

$\sum_{q=1}^{Q_{j}} \sum_{\tilde{t} \cong \widetilde{E F T}_{j}}^{L \widetilde{F T}} x_{j q \tilde{t} s}=1 \quad \forall j \in J, \forall s \in S$

$\sum_{q=1}^{Q_{i}} \sum_{\tilde{t} \cong \widetilde{E F T}_{i}}^{L \widetilde{F} T_{i}} \tilde{t}_{i q \tilde{t} s} \leq \sum_{q=1}^{Q_{j}} \sum_{\tilde{t} \cong \widetilde{E F T}_{j}}^{L \widetilde{F T} T_{j}}\left(\tilde{t}-\tilde{d}_{j q}\right) \cdot x_{j q \tilde{t} s} \quad \forall j \in J, \forall i \in P_{j}, \forall s \in S$

$\sum_{j=2}^{J-1} \sum_{q=1}^{Q_{j}} k r_{j q r s} \cdot \sum_{\tilde{\tau} \cong \tilde{t}}^{\tilde{t}+\tilde{d}_{j q}-1} x_{j q \tilde{\tau} s} \leq \widetilde{K R}_{r s} \quad \forall r \in R, \forall \tilde{t} \in T, \forall s \in S$

$\sum_{j=2}^{J-1} \sum_{q=1}^{Q_{j}} k n_{j q n s} \cdot \sum_{\tilde{t} \cong \widetilde{E F T}_{j}}^{L \widetilde{F T} T_{j}} x_{j q \tilde{t} s} \leq \widetilde{K N}_{n s} \quad \forall n \in N, \forall s \in S$

$x_{j q \tilde{t} s} \in\{0,1\} \quad \forall j \in J, \quad q \in Q_{j}, \quad \tilde{t} \in\left\{\widetilde{E F T_{j}}, \widetilde{L F T} T_{j}, \quad \forall s \in S\right.$

$\widetilde{K R}_{r s}, \widetilde{K N}_{n s} \geq 0$ and integer

$\forall r \in R, \forall n \in N, \forall s \in S$

The first goal in Eq. (1) aims to reduce expected value of the uncertain project makespan which corresponds to a fuzzy completion time of the dummy ending activity. In the second goal, expected value of the total cost resulting from the usage of both renewable and nonrenewable resources is minimized in Eq. (2). It should be highlighted here that since the nonrenewable resource is defined in terms of the monetary value (project funds or budget), availability level or usage amount of this resource type is considered only in Eq. (2). On the other hand, fuzzy usage costs of the renewable resources are multiplied by their usage amounts/availability levels to obtain a similar monetary value. In most of the real-life project scheduling cases, project managers desire to decrease usage costs of the renewable/nonrenewable project resources as well as minimizing the project makespan. However, minimizing the project makespan may need to utilize more resources which cause higher total cost. On the other hand, usage of less amount of resources may cause longer project makespan. For that reason, a compromise solution should be found by taking account the trade-off between project makespan and total cost. Actually, these are the most frequently encountered conflicting objectives in RIPSP applications. The constraint set in Eq. (3) ensures that each activity can be executed under a single mode in each scenario. Moreover, each activity should be completed at a fuzzy time which is ranged between its fuzzy earliest and latest finishing times. In other words, only one execution mode and fuzzy time can be assigned to each project activity under each scenario. According to the constraint set in Eq. (4), precedence relations among the project activities should be satisfied under each scenario. Actually, fuzzy durations and starting/completion times of the activities make this precedence constraint much more complicated when compared to its deterministic version. Because, fuzzy completion time of an activity may intersect with fuzzy starting times of its successors. Because of these overlapped 
fuzzy numbers, precedence relations among the project activities may not be satisfied explicitly. This problematic issue has already mentioned by Subulan (2019 \& 2020) and defined as one of the risky situations in fully uncertain project scheduling applications. Actually, standard fuzzy arithmetic based solution approaches may cause these risky situations. This problematic issue can be overcome by making use of the proposed bound \& decomposition based solution approach without using standard fuzzy arithmetic operations and fuzzy ranking techniques directly. Constraint set in Eq. (5) is related to the capacity limitations of the renewable project resources. Apart from the classical RCPSPs, availability/capacity levels for different types of renewable resources are defined as fuzzy-stochastic decision variables. For that reason, with the help of the second objective function given in Eq. (2), this constraint set also provides minimum renewable resource utilization. According to Eq. (5), the capacity/availability level of each renewable resource type cannot be exceeded at each fuzzy time period under each scenario. Similarly, capacity restrictions on the non-renewable project resources are formulated in Eq. (6). In other words, availability level of each non-renewable resource cannot be surpassed throughout the project duration in each scenario. It should be noted here that when the standard fuzzy arithmetic based solution approaches are implemented, capacity unavailability risks may also arise for these renewable/non-renewable project resources during the project implementation phases (Subulan, 2019 \& 2020). Furthermore, by adding the scenario index $(s \in S)$ to the relevant fuzzy-stochastic decision variables on the resource availability levels, stochastic nature of the project parameters/inputs can be simply reflected to the project schedules (fuzzy completion times of the activities). More specifically, the required availability level of each project resource can be acquired under different scenarios. However, it should be mentioned here that such a scenario-based stochastic programming approach may result increases in the problem dimension due to the addition of this scenario index $(s \in S)$ to the all model variables. Actually, this is the main disadvantage of this approach. In Eq. (7), fuzzy completion times of the project activities are defined as binary integer variables. The nonpreemption of the project activities can also be guaranteed by means of Eqs. (3) and (7). Finally, fuzzy-stochastic availability levels of the renewable/non-renewable project resources are also defined as general integer variables in Eq. (8).

\subsection{Constraint programming (CP) model formulation of the multi-objective FS-MRIPSP}

Similarly, CP model formulation is given by Eqs. (9) - (14) by using the mathematical nomenclature in Tables $2-3$. Indeed, CP model of the FS-MRIPSP is denoted by making use of the Optimization Programming Language (OPL) (IBM, 2017). As shown in Table 3, some additional special variables are also used in the OPL. Actually, the first interval variable represents an interval of time during a project activity execution under different scenarios. On the other hand, the second one is an optional interval variable in which one can determine not to consider them in the solution project schedule. In other words, we need to define this optional interval variable due to the alternative/different modes for executing a given project activity. The mode selection is generally represented by an exclusive alternative between intervals. The interval variable $\tilde{Y}_{j q s}$ is defined with an option "optional"; that is, one can decide not to consider them in the solution project schedule. Actually, these interval variables are provided to express a fuzzy project schedule in the OPL (Okubo et al., 2015).

Table 3 Additional mathematical notation for the CP formulation of the FS-MRIPSP

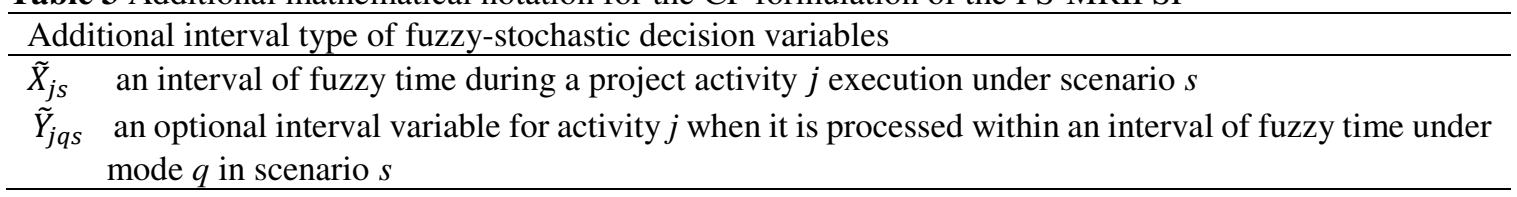


Minimize EV(Project Makespan) $\tilde{Z}_{1} \cong \sum_{S=1}^{S} \pi_{s}$. endOf $\left(\tilde{X}_{J S}\right)$

Minimize EV(Total Resource Costs) $\tilde{Z}_{2} \cong \sum_{n=1}^{N} \sum_{s=1}^{s} \pi_{s} \cdot \widetilde{K N}_{n s}+\sum_{r=1}^{R} \sum_{s=1}^{s} \pi_{s} \cdot \widetilde{K R}_{r s} \cdot \tilde{C}_{r}$

Subject to;

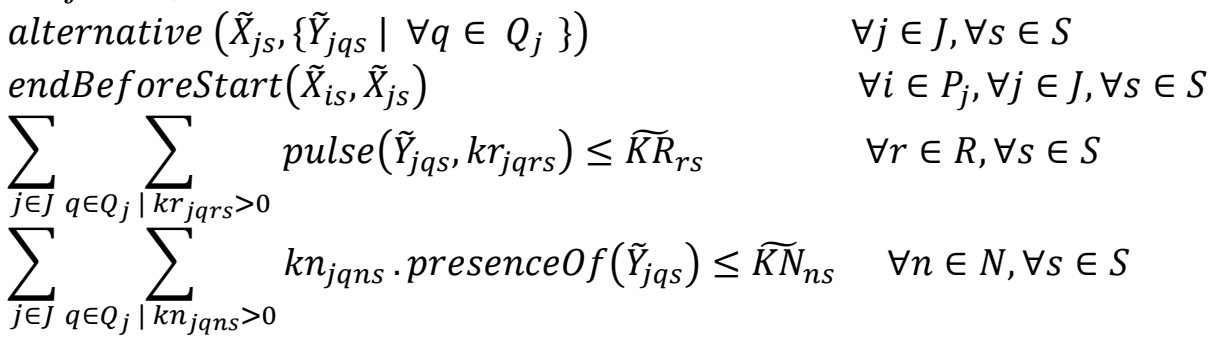

It should be emphasized that all of the previous $\mathrm{CP}$ models for project scheduling problems usually used these interval type variables under deterministic environments (Liess \& Michelon, 2008; Berthold et al., 2010; Liu \& Song, 2011; Okubo et al., 2015). In other words, deterministic time intervals were commonly defined in the available literature. Unlike the former studies, these interval variables are described under fuzziness throughout this paper. In fact, fuzzy time intervals are described because of the fuzzy activity durations. Therefore, the proposed $\mathrm{CP}$ model is also able to generate fuzzy project schedules as in the previously formulated ILP model. Apart from these additional interval variables, the proposed CP model also utilizes some tuples and special functions like endOf, alternative, endBeforeStart, pulse and presenceOf. A brief description of each function will be given in this section. For more details, please refer to IBM OPL Language User's Manual (2017). Firstly, function endOf returns the fuzzy end time of a given interval variable $\left(\tilde{X}_{J S}\right)$ for dummy ending activity. Thus, objective function in Eq. (9) minimizes the expected value of project makespan and correspond to the objective function given in Eq. (1). The second objective function in Eq. (10) which aims to minimize expected value of total resource usage costs remains the same as in Eq. (2). In Eq. (11), alternative function is utilized for mode selection of each project activity. Let $\tilde{X}_{j s}$ and $\tilde{Y}_{j q s} \mid \forall q \in Q_{j}$ be fuzzy intervals. The constraint, i.e., alternative $\left(\tilde{X}_{j s},\left\{\tilde{Y}_{j q s} \mid \forall q \in Q_{j}\right\}\right)$ enables to model an exclusive alternative between $\left\{\tilde{Y}_{j q s} \mid \forall q \in Q_{j}\right\}$. It should also be emphasized here that when a fuzzy interval variable is used in the solution schedule, it is named as present, otherwise it is called absent. If a fuzzy interval, i.e., $\tilde{X}_{j s}$ is present, then exactly one fuzzy interval $\tilde{Y}_{j q s} \mid \forall q \in Q_{j}$ is present and $\tilde{X}_{j s}$ begins and ends together with this selected mode. Therefore, constraint in Eq. (11) implies that each project activity is processed under only one mode and it is executed only once under each scenario. Actually, this constraint corresponds to the constraint sets in Eqs. (3) and (7) in the previous ILP formulation. The constraint function endBeforeStart $\left(\tilde{X}_{i s}, \tilde{X}_{j s}\right)$ states that the end of a given fuzzy interval variable, i.e., $\tilde{X}_{i s}$ is less than or equal to the start of a given fuzzy interval variable $\widetilde{X}_{j s}$. Thus, constraint (12) guarantees the precedence relations among the project activities and corresponds to the constraint set in Eq. (4). Constraint (13) represents the capacity restrictions for all of the renewable resources; where pulse $\left(\tilde{Y}_{j q s}, k r_{j q r s}\right)$ is an elementary cumulative function and its value is equal to $k r_{j q r s}$ during fuzzy time interval in which optional interval $\tilde{Y}_{j q s} \forall q \in Q_{j}$ is present. In detail, a fuzzy interval increases the cumulated resource usage at its start time and decreases it when it releases this resource at its end time by making use of pulse function. In other words, pulse function increases or decreases the level of the resource utilization by a given amount, i.e., $k r_{j q r s}$ for the length of a given fuzzy interval variable $\tilde{Y}_{j q s} \forall q \in Q_{j}$ under each 
scenario. This constraint set in Eq. (13) also corresponds to Eq. (5) in the proposed ILP formulation. Unlike this renewable resource capacity restriction, presence $O f$ function is utilized for satisfying the capacity limitations of non-renewable resources in Eq. (14). Here, pulse function should not employed since the capacity constraints of non-renewable resources should not be satisfied for the length of a given fuzzy interval. Instead, capacity levels of the nonrenewable resources should not be exceeded throughout the entire project duration in each scenario. To do this, the function presenceOf is used which returns " 1 " when a given fuzzy interval $\tilde{Y}_{j q s} \forall q \in Q_{j}$ is present. Otherwise, it returns " 0 " when fuzzy interval $\tilde{Y}_{j q s} \forall q \in Q_{j}$ is absent. More specifically, presence $O f$ function returns the Boolean expression that represents the presence statue of a fuzzy interval variable, i.e., $\widetilde{Y}_{j q s} \forall q \in Q_{j}$. If activity $j$ is executed under mode $q$ in scenario $s$, fuzzy interval $\tilde{Y}_{j q s}$ must be present and its value is taken as " 1 "; otherwise, this function returns the value of "0". Constraint set in Eq. (14) also corresponds to Eq. (6) in the ILP model.

\section{The proposed ILP \& CP-based transformations with bound \& decomposition principle}

In this section, ILP \& CP-based transformation approaches with a bound \& decomposition principle are presented in order to convert the FS-MRIPSP into its crisp equivalent form. As mentioned previously, in order to avoid the direct usage of standard fuzzy arithmetic operations and fuzzy ranking techniques (Sotoudeh-Anvari, 2020; Subulan, 2020), we preferred to implement a bound \& decomposition principle of Jayalakshmi \& Pandian (2012). Because, this approach does not apply fuzzy arithmetic operations directly and it does not require the usage of any fuzzy ranking function and additional auxiliary fuzzy variables. It mainly depends on the decomposition of any given fuzzy mathematical programming problem (FMPP) into three or four crisp problems with additional bounded variables and constraints while dealing with triangular and trapezoidal fuzzy numbers, respectively (Baykasoğlu \& Subulan, 2015). Since this approach divides the FMPP into three or four crisp equivalent problems, model dimension, i.e., the number of decision variables and constraints do not increase excessively because of this decomposition stage. In fact, this is one of its important advantage for solving such a combinatorial fuzzy optimization problem, i.e., FS-MRIPSP. On the other hand, standard fuzzy arithmetic based transformation approaches may cause significant increases in the model dimension which makes the solution of the problem much more difficult. The details of this approach can be summarized through the following sub-sections.

\subsection{ILP-based transformation approach with a bound \& decomposition principle}

When the aforementioned fuzzy project parameters and variables are represented by triangular fuzzy numbers, the previous ILP formulation given by Eqs. (1) - (8) can be reformulated as in the following Eqs. (15) - (22). Indeed, additional indices, i.e., $l, m$ and $u$ are added to all fuzzy parameters and decision variables so as to represent the core, left and right margins of the triangular fuzzy numbers, respectively.

$$
\begin{aligned}
\text { Minimize }\left(Z 1_{l}, Z 1_{m}, Z 1_{u}\right)= & \sum_{\left(t_{l}, t_{m}, t_{u}\right)=\left(E F T_{J l}, E F T_{J m}, E F T_{J u}\right)}^{\left(L F T_{J l}, L F T_{J m}, L F T_{J u}\right)} \sum_{s=1}^{S} \pi_{s} \cdot\left(t_{l}, t_{m}, t_{u}\right) \cdot x_{J 1\left(t_{l}, t_{m}, t_{u}\right) s} \\
\text { Minimize }\left(Z 2_{l}, Z 2_{m}, Z 2_{u}\right)= & \sum_{n=1}^{S} \sum_{s=1}^{S} \pi_{s} \cdot\left(K N_{n s l}, K N_{n s m}, K N_{n s u}\right)+ \\
& \sum_{r=1}^{R} \sum_{s=1}^{s} \pi_{s} \cdot\left(K R_{r s l}, K R_{r s m}, K R_{r s u}\right) \cdot\left(C_{r l}, C_{r m}, C_{r u}\right)
\end{aligned}
$$




$$
\begin{aligned}
& \sum_{q=1}^{Q_{j}} \sum_{\left(t_{l}, t_{m}, t_{u}\right)=\left(E F T_{j l}, E F T_{j m}, E F T_{j u}\right)}^{\left(L F T_{j l}, L F T_{j m}, L F T_{j u}\right)} x_{j q\left(t_{l}, t_{m}, t_{u}\right) s}=1 \quad \forall j \in J, \forall s \in S \\
& \sum_{q=1}^{Q_{i}} \sum_{\left(t_{l}, t_{m}, t_{u}\right)=\left(E F T_{i l}, E F T_{i m}, E F T_{i u}\right)}^{\left(L F T_{i l}, L F T_{i m}, L F T_{i u}\right)}\left(t_{l}, t_{m}, t_{u}\right) \cdot x_{i q\left(t_{l}, t_{m}, t_{u}\right) s} \\
& \leq \sum_{q=1}^{Q_{j}} \sum_{\left(t_{l}, t_{m}, t_{u}\right)=\left(E F T_{j l}, E F T_{j m}, E F T_{j u}\right)}^{\left(L F T_{j l}, L F T_{j m}, L F T_{j u}\right)}\left\{\left(t_{l}, t_{m}, t_{u}\right) \ominus\left(d_{j q l}, d_{j q m}, d_{j q u}\right)\right\} \cdot x_{j q\left(t_{l}, t_{m}, t_{u}\right) s} \\
& \forall j \in J, \forall i \in P_{j}, \forall s \in S \\
& \sum_{j=2}^{J-1} \sum_{q=1}^{Q_{j}} k r_{j q r s} . \sum_{\left(\tau_{l}, \tau_{m}, \tau_{u}\right)=\left(t_{l}, t_{m}, t_{u}\right)}^{\left(t_{l}, t_{m}, t_{u}\right) \oplus\left(d_{j q l}, d_{j q m}, d_{j q u}\right)-1} x_{j q\left(\tau_{l}, \tau_{m}, \tau_{u}\right) s} \leq\left(K R_{r s l}, K R_{r s m}, K R_{r s u}\right) \\
& \sum_{j=2}^{J-1} \sum_{q=1}^{Q_{j}} k n_{j q n s} . \sum_{\left(t_{l}, t_{m}, t_{u}\right)=\left(E F T_{j l}, E F T_{j m}, E F T_{j u}\right)}^{\left(L F T_{j l}, L F T_{j m}, L F T_{j u}\right)} x_{j q\left(t_{l}, t_{m}, t_{u}\right) s} \leq\left(K N_{n s l}, K N_{n s m}, K N_{n s u}\right) \\
& \forall r \in R, \forall\left(t_{l}, t_{m}, t_{u}\right) \in T, \forall s \in S \\
& x_{j q\left(t_{l}, t_{m}, t_{u}\right) s} \in\{0,1\} \\
& \forall j \in J, q \in Q_{j}, \forall s \in S,\left(t_{l}, t_{m}, t_{u}\right) \in\left\{\left(E F T_{j l}, E F T_{j m}, E F T_{j u}\right),\left(L F T_{j l}, L F T_{j m}, L F T_{j u}\right)\right\}
\end{aligned}
$$

By applying the bound \& decomposition principle of Jayalakshmi \& Pandian (2012), the above fuzzy-stochastic model is first decomposed into the middle level, upper level and lower level problems (Decomposition stage). Afterwards, these crisp equivalent problems should be solved separately and fuzzy efficient solutions of the FS-MRIPSP can be generated by utilizing their solutions as bounded variables and constraints. It should be mentioned here that middle level problem in which at least one decision variable of the middle level problem exists into the constraints is solved at first. The crisp equivalent form of the middle level problem can be formulated as in the following Eqs. (23) - (30).

Minimize $Z 1_{m}=\sum_{t_{m}=E F T_{J m}}^{L F T_{J m}} \sum_{s=1}^{S} \pi_{s} \cdot t_{m} \cdot x_{J 1 t_{m} s}$
Minimize $Z 2_{m}=\sum_{n=1}^{N} \sum_{s=1}^{S} \pi_{s} \cdot K N_{n s m}+\sum_{r=1}^{R} \sum_{s=1}^{S} \pi_{s} \cdot K R_{r s m} \cdot C_{r m}$

Subject to;

$\sum_{q=1}^{Q_{j}} \sum_{t_{m}=E F T_{j m}}^{L F T_{j m}} x_{j q t_{m} s}=1 \quad \forall j \in J, \forall s \in S$

$\sum_{q=1}^{Q_{i}} \sum_{t_{m}=E F T_{i m}}^{L F T_{i m}} t_{m} \cdot x_{i q t_{m} s} \leq \sum_{q=1}^{Q_{j}} \sum_{t_{m}=E F T_{j m}}^{L F T_{j m}}\left\{t_{m}-d_{j q m}\right\} \cdot x_{j q t_{m} s} \quad \forall j \in J, \forall i \in P_{j}, \forall s \in S$

$\sum_{j=2}^{J-1} \sum_{\substack{q=1 \\ Q_{j}}}^{Q_{j}} k r_{j q r s} \cdot \sum_{\substack{\tau_{m}=t_{m} \\ L F T_{j m}}}^{t_{m}+d_{j q m}-1} x_{j q \tau_{m} s} \leq K R_{r s m}$

$\forall r \in R, \forall t_{m} \in T, \forall s \in S$

$\sum_{j=2} \sum_{q=1} k n_{j q n s} \cdot \sum_{t_{m}=E F T_{j m}} x_{j q t_{m} s} \leq K N_{n s m}$

$\forall n \in N, \forall s \in S$ 
$x_{j q t_{m} s} \in\{0,1\}$

$K R_{r s m}, K N_{n s m} \geq 0$ and integer $\forall j \in J, q \in Q_{j}, \forall s \in S, t_{m} \in\left\{E F T_{j m}, L F T_{j m}\right\}$

$\forall r \in R, \forall n \in N, \forall s \in S$

After generating the solution of the above middle level problem, the upper level problem in which at least one decision variable of this upper level problem occurs into the constraints and should not take place in the middle level problem is formulated as in Eqs. (31) - (43). Before solving this upper level problem, values of the decision variables are replaced with the values provided by the middle level problem. In other words, outputs of the middle level problem are used as input data while formulating the bound constraints of the upper level problem (see in Eqs. 39 -41). Additionally, this upper level problem should also satisfy the bounded constraints in Eqs. (33) - (34) which are related to the core and right margins of fuzzy objective values.

$\begin{array}{ll}\text { Minimize } & Z 1_{u}=\sum_{t_{u}=E F T_{J u}}^{L F T_{J u}} \sum_{s=1}^{S} \pi_{s} \cdot t_{u} \cdot x_{J 1 t_{u} s} \\ \text { Minimize } \quad Z 2_{u}=\sum_{n=1}^{N} \sum_{s=1}^{S} \pi_{s} \cdot K N_{n s u}+\sum_{r=1}^{R} \sum_{s=1}^{S} \pi_{s} \cdot K R_{r s u} \cdot C_{r u}\end{array}$

Subject to;

$Z 1_{u} \geq Z 1_{m}^{*}$

$Z 2_{u} \geq Z 2_{m}^{*}$

$\sum_{q=1}^{Q_{j}} \sum_{t_{u}=E F T_{j u}}^{L F T_{j u}} x_{j q t_{u} s}=1$

$\forall j \in J, \forall s \in S$

$\sum_{q=1}^{Q_{i}} \sum_{t_{u}=E F T_{i u}}^{L F T_{i u}} t_{u} \cdot x_{i q t_{u} s} \leq \sum_{q=1}^{Q_{j}} \sum_{t_{u}=E F T_{j u}}^{L F T_{j u}}\left\{t_{u}-d_{j q u}\right\} . x_{j m t_{u} s} \quad \forall j \in J, \forall i \in P_{j}, \forall s \in S$

$\sum^{J-1} \sum_{j}^{Q_{j}} \quad \sum_{j}+d_{j q u}-1$

$\sum_{j=2} \sum_{\substack{q=1 \\ J-1}} k r_{j q r s} \cdot \sum_{\substack{\tau_{u}=t_{u} \\ L F T_{j u}}} x_{j q \tau_{u} s} \leq K R_{r s u} \quad \forall r \in R, \forall t_{u} \in T, \forall s \in S$

$\sum_{j=2}^{J-1} \sum_{q=1}^{Q_{j}} k n_{j q n s} \cdot \sum_{t_{u}=E F T_{j u}}^{L F T_{j u}} x_{j q t_{u} s} \leq K N_{n s u}$

$\forall n \in N, \forall s \in S$

$t_{u} \cdot x_{j q t_{u} s} \geq t_{m}^{*} \cdot x_{j q^{*}} t_{m}^{*} s$

$$
\begin{gathered}
\forall j \in J, \forall s \in S, \quad q=q^{*} \\
\forall r \in R, \forall s \in S \\
\forall n \in N, \forall s \in S \\
\forall j \in J, q \in Q_{j}, \quad \forall s \in S, t_{u} \in\left\{E F T_{j u}, L F T_{j u}\right\} \\
\forall r \in R, \forall n \in N, \forall s \in S
\end{gathered}
$$$$
K N_{\text {su }} \geq K N^{*}
$$$$
x_{j q t_{u} s} \in\{0,1\}
$$$$
K R_{\text {rsu }}, K N_{\text {nsu }} \geq 0 \text { and integer }
$$

According to constraint sets in Eqs. (33) - (34), objective function values of the upper level problem should be greater than the objective values of the middle level problem. Actually, this logical constraints enables to form of a triangular fuzzy objective value for the stated FSMRIPSP. Constraint set in Eq. (39) ensures that right margins of the fuzzy completion times of the project activities should be greater than the core or most likely values of these fuzzy completion times under each scenario. Indeed, completion times of the project activities which are obtained from the upper level problem should be larger than their most likely values produced by the middle level problem. In a similar manner, bound constraints are also formulated for the availability levels of both renewable and non-renewable project resources as in Eqs. (40) - (41). Actually, these are the bound constraints added to the upper level problem. 
After solving the middle and upper level problems, lower level problem in which at least one decision variable of this lower level problem occurs into the constraints and should not take place in both of the middle and upper level problems is formulated in Eqs. (44) - (57).

Minimize $\quad Z 1_{l}=\sum_{t_{l}=E F T_{J l}}^{L F T_{J l}} \sum_{S=1}^{S} \pi_{s} \cdot t_{l} \cdot x_{J 1 t_{l} S}$

Minimize $\quad Z 2_{l}=\sum_{n=1}^{N} \sum_{s=1}^{S} \pi_{s} . K N_{n s l}+\sum_{r=1}^{R} \sum_{s=1}^{S} \pi_{s} . K R_{r s l} . C_{r l}$

Subject to;

$$
\begin{aligned}
& Z 1_{l} \leq Z 1_{m}^{*} \\
& Z 2_{l} \leq Z 2_{m}^{*} \\
& Q_{j} \quad L F T_{j l} \\
& \sum_{q=1} \sum_{t_{l}=E F T_{j l}} x_{j q t_{l} s}=1 \\
& \sum_{q=1}^{Q_{i}} \sum_{t_{l}=E F T_{i l}}^{L F T_{i l}} t_{l} \cdot x_{i q t_{l} s} \leq \sum_{q=1}^{Q_{j}} \sum_{t_{l}=E F T_{j l}}^{L F T_{j l}}\left\{t_{l}-d_{j q l}\right\} \cdot x_{j m t_{l} s} \quad \forall j \in J, \forall i \in P_{j}, \forall s \in S \\
& \sum_{j=2}^{J-1} \sum_{q=1}^{Q_{j}} k r_{j q r s} \cdot \sum_{\tau_{l}=t_{l}}^{t_{l}+d_{j q l}-1} x_{j q \tau_{l} s} \leq K R_{r s} \\
& \sum_{j=2}^{J-1} \sum_{q=1}^{Q_{j}} k n_{j q n s} \cdot \sum_{t_{l}=E F T_{j l}}^{L F T_{j l}} x_{j q t_{l} s} \leq K N_{n s l} \\
& t_{l} \cdot x_{j q t_{l} s} \leq t_{m}^{*} \cdot x_{j q^{*} t_{m}^{*} s} \\
& \forall r \in R, \forall t_{l} \in T, \forall s \in S \\
& \forall n \in N, \forall s \in S \\
& \forall j \in J, \forall s \in S, \quad q=q^{*} \\
& \sum_{q \neq q^{*}}^{Q_{j}} \sum_{t_{l}=E F T_{j l}}^{L F T_{j l}} t_{l} \cdot x_{j q t_{l} S}=0 \\
& K R_{r s l} \leq K R_{r s m}^{*} \\
& K N_{n s l} \leq K N_{n s m}^{*} \\
& x_{j q t_{l} s} \in\{0,1\} \\
& K R_{r s l}, K N_{n s l} \geq 0 \text { and integer } \\
& \forall j \in J, q \in Q_{j}, \forall s \in S, t_{l} \in\left\{E F T_{j l}, L F T_{j l}\right\} \\
& \forall j \in J, \forall s \in S
\end{aligned}
$$

In a similar way, before solving the lower level problem, values of the decision variables are replaced with the obtained solutions from the middle and upper level problems. The lower level problem should also satisfy the bound constraints given in Eqs. (46) - (47) and (52) - (55). Constraint sets in Eqs. (44) - (45) guarantee that objective function values of the lower level problem should be equal or less than the objective values of the middle level problem. In fact, this logical bound constraints makes sure that fuzzy objective values will take a form of triangular fuzzy numbers. Constraint set in Eq. (52) satisfies that left margins of the fuzzy completion times of the project activities should be lower than the core or most likely values of these fuzzy completion times under each scenario. It should be highlighted here that the same execution mode $\left(q^{*} \in Q_{j}\right)$ which is provided by the solution middle level problem should be utilized in these bound constraints (Eqs. 39 and 52) of the upper and lower level problems, respectively. Because, a project activity should be completed at a fuzzy time with the same execution mode under each scenario. In other words, different execution modes cannot be selected for the same project activity under the same scenario in the lower, middle and upper level problems. Indeed, constraint set in Eq. (39) of the upper level problem can meet this 
statement by oneself. On the other hand, an additional logical constraint in Eq. (53) is also required to satisfy this condition in the lower level problem. Because, on the contrary to the constraint set in Eq. (39), Eq. (52) does not guarantee the selection of the same execution mode in the middle level problem by oneself. By formulating an additional logical constraint as in Eq. (53), a different execution mode which is not found by the solution of middle level problem cannot be chosen for any project activity under the same scenario. Fortunately, this logical restriction can be automatically satisfied and does not needed by the CP model of the lower level problem. In Eqs. (54) - (55), bound constraints are also formulated so as to obtain the optimistic values of the resources' availability levels. When these middle, upper and lower level problems are solved sequentially, their separate solutions are brought together to generate fuzzy efficient solutions, i.e., project schedules, $x_{j q}\left(t_{l}, t_{m}, t_{u}\right) s$ and resources' availability levels, $\left(K R_{r s l}, K R_{r s m}, K R_{r s u}\right),\left(K N_{n s l}, K N_{n s m}, K N_{n s u}\right)$ under each scenario with the expected values of fuzzy project makespan, $\left(Z 1_{l}, Z 1_{m}, Z 1_{u}\right)$ and total resource usage costs $\left(Z 2_{l}, Z 2_{m}, Z 2_{u}\right)$.

\subsection{CP-based transformation approach with a bound \& decomposition principle}

Similar to the ILP formulations given in Eqs. (15) - (22), the previously formulated CP model in Eqs. (9) - (14) can be reformulated as in the following Eqs. (58) - (63) when dealing with fuzzy project parameters and variables. Actually, an additional index is incorporated into this formulation to represent the core, left and right margins of the triangular fuzzy numbers.

$$
\begin{aligned}
& \text { Minimize }\left(Z 1_{l}, Z 1_{m}, Z 1_{u}\right)=\sum_{S=1}^{S} \pi_{s} . \text { endof }\left(X_{J s l}, X_{J s m}, X_{J s u}\right) \\
& \text { Minimize }\left(Z 2_{l}, Z 2_{m}, Z 2_{u}\right)=\sum_{n=1}^{N} \sum_{s=1}^{S} \pi_{s} \cdot\left(K N_{n s l}, K N_{n s m}, K N_{n s u}\right)+ \\
& \qquad \sum_{r=1}^{R} \sum_{s=1}^{S} \pi_{s} .\left(K R_{r s l}, K R_{r s m}, K R_{r s u}\right) .\left(C_{r l}, C_{r m}, C_{r u}\right)
\end{aligned}
$$

Subject to;

$$
\begin{aligned}
& \text { alternative }\left(\left(X_{j s l}, X_{j s m}, X_{j s u}\right),\left\{\left(Y_{j q s l}, Y_{j q s m}, Y_{j q s u}\right) \mid q \in Q_{j}\right\}\right) \quad \forall j \in J, \forall s \in S \\
& \text { endBeforeStart }\left(\left(X_{i s l}, X_{i s m}, X_{i s u}\right),\left(X_{j s l}, X_{j s m}, X_{j s u}\right)\right) \quad \forall i \in P_{j}, \forall j \in J, \forall s \in S
\end{aligned}
$$

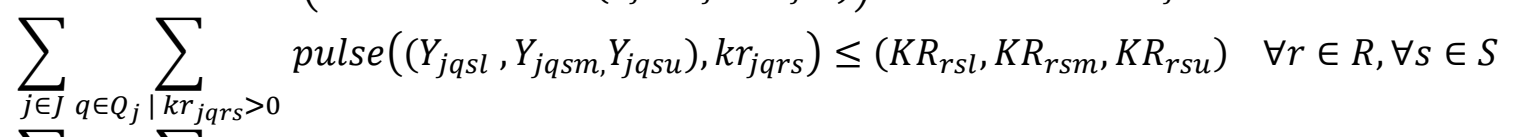

$$
\begin{aligned}
& \sum_{j \in J} \sum_{q \in Q_{j} \mid k n_{j q n s}>0} k n_{j q n s} \cdot \text { presenceOf }\left(\left(Y_{j q s l}, Y_{j q s m}, Y_{j q s u}\right)\right) \leq\left(K N_{n s l}, K N_{n s m}, K N_{n s u}\right) \\
& \forall n \in N, \forall s \in S
\end{aligned}
$$

Similarly, CP model of the crisp equivalent middle level problem can be formulated as in the following Eqs. (64) - (70). It should be noted here that only the core or most likely values of the fuzzy activity durations, completions times and availability levels are utilized in this middle level problem formulation.

$$
\begin{array}{ll}
\text { Minimize } & Z 1_{m}=\sum_{s=1}^{S} \pi_{s} \text { endOf }\left(X_{J s m}\right) \\
\text { Minimize } & Z 2_{m}=\sum_{n=1}^{N} \sum_{s=1}^{S} \pi_{s} \cdot K N_{n s m}+\sum_{r=1}^{R} \sum_{s=1}^{S} \pi_{s} \cdot K R_{r s m} \cdot C_{r m}
\end{array}
$$

Subject to;

$$
\operatorname{endOf}\left(X_{J s m}\right) \leq L F T_{J m} \quad \forall s \in S
$$




$$
\begin{array}{lc}
\text { alternative }\left(X_{j s m},\left\{Y_{j q s m} \mid q \in Q_{j}\right\}\right) & \forall j \in J, \forall s \in S \\
\text { endBeforeStart }\left(X_{i s m}, X_{j s m}\right) & \forall i \in P_{j}, \forall j \in J, \forall s \in S \\
\sum_{j \in J} \sum_{q \in Q_{j} \mid k r_{j q r s}>0} \operatorname{pulse}\left(Y_{j q s m}, k r_{j q r s}\right) \leq K R_{r s m} & \forall r \in R, \forall s \in S \\
\sum_{j \in J} \sum_{q \in Q_{j}} k n_{j q n s}, \text { presenceOf }\left(Y_{j q s m}>0\right. &
\end{array}
$$

It should be emphasized here that completion time of the dummy ending activity is controlled by making use of the constraint set in Eq. (66) to satisfy that end of the finishing time interval should be equal or lower than the previously computed latest finish time under each scenario. Therefore, this CP model formulation will also be consistent with the ILP model which uses the earliest and latest finishing time information. After solving this crisp middle problem by making use of the constraint programming solver of IBM ILOG OPL Optimization Studio, CP model formulation of the crisp equivalent upper level problem can be constructed as in Eqs. (71) - (82). Only the pessimistic values or right margins of fuzzy activity durations, completion times and availability levels are involved in this formulation.

$$
\begin{array}{ll}
\text { Minimize } & Z 1_{u}=\sum_{s=1}^{S} \pi_{s} \cdot \operatorname{endOf}\left(X_{J s u}\right) \\
\text { Minimize } & Z 2_{u}=\sum_{n=1}^{N} \sum_{s=1}^{S} \pi_{s} \cdot K N_{n s u}+\sum_{r=1}^{R} \sum_{s=1}^{S} \pi_{s} \cdot K R_{r s u} \cdot C_{r u}
\end{array}
$$

Subject to;

$Z 1_{u} \geq Z 1_{m}^{*}$

$$
\begin{array}{ll}
\text { endOf }\left(X_{J s u}\right) \leq L F T_{J u} & \forall s \in S \\
Z 2_{u} \geq Z 2_{m}^{*} &
\end{array}
$$

alternative $\left(X_{j s u},\left\{Y_{j q s u} \mid q=q *\right\}\right)$

$\forall j \in J, \forall s \in S$

endBeforeStart $\left(X_{i s u}, X_{j s u}\right)$

$\sum_{j \in J} \sum_{q \in Q_{j} \mid k r_{j q r s}>0} p u l s e\left(Y_{j q s u}, k r_{j q r s}\right) \leq K R_{r s u}$

$$
\forall i \in P_{j}, \forall j \in J, \forall s \in S
$$$$
\forall r \in R, \forall s \in S
$$

$\sum_{j \in J} \sum_{q \in Q_{j} \mid k n_{j q n s}>0} k n_{j q n s} \cdot$ presenceOf $\left(Y_{j q s u}\right) \leq K N_{n s u} \quad \forall n \in N, \forall s \in S$

$\operatorname{endOf}\left(Y_{j q s u} \mid q=q *\right) \geq \operatorname{endO} f\left(Y_{j q s m}^{*}\right)$

$$
\begin{aligned}
& \forall j \in J, \forall s \in S \\
& \forall r \in R, \forall s \in S \\
& \forall n \in N, \forall s \in S
\end{aligned}
$$

The above CP model also includes similar bound constraints in Eqs. (73) - (65) and (80) (82). Actually, these bound constraints related to the fuzzy objective values are the same with the previous ILP formation of the upper level problem in Eqs. (33) - (34). Apart from the ILP formulation, end $O f$ function is utilized in some bound constraint of the $\mathrm{CP}$ model to satisfy that the right margins of the fuzzy completion times should be greater than their most likely or core values (see Eq. 80). Moreover, pessimistic completion time of the dummy ending activity should also be equal or lower than the right margin of its fuzzy latest finishing time (see Eq. 74). It is clear in Eqs. (76) and (80) that the same execution mode which is previously specified by the solution of middle level problem must be selected for each project activity under each scenario. Similarly, CP model formulation of the crisp equivalent lower level problem can also be presented as in Eqs. (83) - (94). 
Minimize $\quad Z 1_{l}=\sum_{s=1}^{S} \pi_{s}$. endof $\left(X_{J s l}\right)$
Minimize $\quad Z 2_{l}=\sum_{n=1}^{N} \sum_{s=1}^{S} \pi_{s} \cdot K N_{n s l}+\sum_{r=1}^{R} \sum_{s=1}^{S} \pi_{s} \cdot K R_{r s l} \cdot C_{r l}$

Subject to;

$Z 1_{l} \leq Z 1_{m}^{*}$

$Z 2_{l} \leq Z 2_{m}^{*}$

$\forall s \in S$

alternative $\left(X_{j s l},\left\{Y_{j q s l} \mid q=q *\right\}\right)$

$\forall j \in J, \forall s \in S$

endBeforeStart $\left(X_{i s l}, X_{j s l}\right)$

$\forall i \in P_{j}, \forall j \in J, \forall s \in S$

$\sum_{j \in J} \sum_{q \in Q_{j} \mid k r_{j q r s}>0}$ pulse $\left(Y_{j q s l}, k r_{j q r s}\right) \leq K R_{r s l}$

$\forall r \in R, \forall s \in S$

$\sum_{j \in J} \sum_{q \in Q_{j} \mid k n_{j q n s}>0} k n_{j q n s} \cdot$ presenceOf $\left(Y_{j q s l}\right) \leq K N_{n s l} \quad \forall n \in N, \forall s \in S$

$\operatorname{endOf}\left(Y_{j q s l} \mid q=q *\right) \leq \operatorname{endO} f\left(Y_{j q s m}^{*}\right)$

$\forall j \in J, \forall s \in S$

$K R_{r s l} \leq K R_{r s m}^{*}$

$K N_{n s l} \leq K N_{n s m}^{*}$

$\forall r \in R, \forall s \in S$

$\forall n \in N, \forall s \in S$

In the above formulation, only the optimistic values or left margins of the fuzzy activity durations, completion times and availability levels are taken into account. After solving the middle, upper and lower level problems sequentially, their solutions are aggregated to produce fuzzy efficient solutions related to (i) project schedules i.e., $\left(X_{j s l}, X_{j s m}, X_{j s u}\right)$ and $\left(Y_{j q s l}, Y_{j q s m}, Y_{j q s u}\right)$, (ii) resources' availability levels, i.e., $\left(K R_{r s l}, K R_{r s m}, K R_{r s u}\right)$ and $\left(K N_{n s l}, K N_{n s m}, K N_{n s u}\right)$ under each scenario with the expected values of the fuzzy objectives $\left(Z 1_{l}, Z 1_{m}, Z 1_{u}\right)$ and $\left(Z 2_{l}, Z 2_{m}, Z 2_{u}\right)$.

\subsection{Illustrative example of classical MRCPSP under mixed fuzzy \& stochastic uncertainties}

This section presents a trivial numerical example of a classical multi-mode resourceconstrained project scheduling problem (MRCPSP) with a single makespan criterion in order to show validity and applicability of the proposed IP \& CP-based transformation approaches. Actually, this example problem is derived based on a deterministic problem instance from the existing literature (Kolisch \& Drexl, 1997). Indeed, that original deterministic problem is extended by incorporating the following fuzzy and stochastic project data. The project is composed of 5 non-dummy activities with 2 modes and they require uncertain amounts of monotype renewable and non-renewable resources whose capacities are also uncertain. The activity-on-node (AON) project network diagram is shown in Fig. 2 with fuzzy activity durations under different modes above/below each node. The fuzzy earliest and latest finishing times (EFT \& LFT) of the project activities are also calculated and presented in the same figure. The start time of the project is certain and set to $t_{0}=(0,0,0)$. The length of the project planning horizon is computed as 24 discrete-time periods by summing the maximum activity durations that are chosen from among all the execution modes. In contrast to the fuzzy activity durations, resource requirements of the project activities and the resources' capacities are considered as stochastic project parameters since they may have randomness in nature. In Table 4 , stochastic data related to the resources' capacities and the resource requirements of the project activities are given with the pre-specified discrete probabilities under each mode. Actually, these project parameters are defined as discrete random variables with known probabilities. In detail, it is assumed that both renewable and non-renewable resources may have low or high capacity levels with a discrete probability during the project realization phase. 


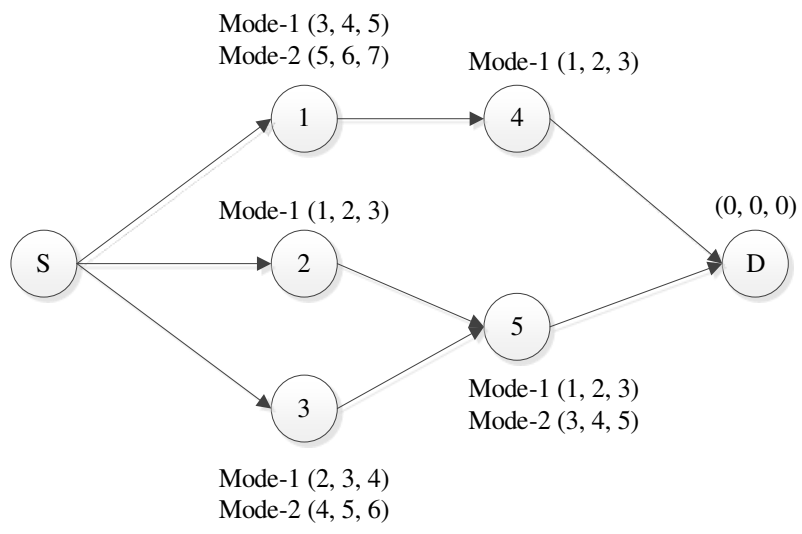

\begin{tabular}{ccc}
\hline Activity & Fuzzy EFT & Fuzzy LFT \\
\hline S & $(0,0,0)$ & $(10,13,16)$ \\
1 & $(3,4,5)$ & $(13,17,21)$ \\
2 & $(1,2,3)$ & $(13,17,21)$ \\
3 & $(2,3,4)$ & $(13,17,21)$ \\
4 & $(4,6,8)$ & $(14,19,24)$ \\
5 & $(3,5,7)$ & $(14,19,24)$ \\
D & $(4,6,8)$ & $(14,19,24)$ \\
\hline
\end{tabular}

Fig. 2 The AON project network diagram with fuzzy durations and the earliest/latest finishing times of activities

Similarly, project activities may require low or high amounts of the resources with respect to the dynamically changing conditions. Based on this random data, 16 different scenarios with equal joint probabilities are generated as depicted in Fig. 3.

Table 4 Stochastic project data with discrete probabilities: Resources' capacities and resource requirements

\begin{tabular}{|c|c|c|c|c|c|c|c|c|}
\hline \multirow{3}{*}{ Project Activities } & \multicolumn{4}{|c|}{ Renewable Resource } & \multicolumn{4}{|c|}{ Non-renewable Resource } \\
\hline & \multicolumn{2}{|c|}{ Low (Pr. 50\%) } & \multicolumn{2}{|c|}{ High (Pr. 50\%) } & \multicolumn{2}{|c|}{ Low (Pr. 50\%) } & \multicolumn{2}{|c|}{ High (Pr. 50\%) } \\
\hline & Mode-1 & Mode-2 & Mode-1 & Mode-2 & Mode-1 & Mode-2 & Mode-1 & Mode-2 \\
\hline$S$ & 0 & $*$ & 0 & $*$ & 0 & $*$ & 0 & $*$ \\
\hline 1 & 2 & 1 & 3 & 3 & 2 & 0.5 & 3 & 1 \\
\hline 2 & 1 & $*$ & 2 & $*$ & 0 & $*$ & 1 & $*$ \\
\hline 3 & 3 & 1 & 4 & 2 & 3 & 1 & 4 & 2 \\
\hline 4 & 1 & $*$ & 2 & $*$ & 0 & $*$ & 1 & $*$ \\
\hline 5 & 2 & 1 & 3 & 2 & 2 & 0.5 & 3 & 1 \\
\hline $\mathrm{D}$ & 0 & $*$ & 0 & $*$ & 0 & $*$ & 0 & $*$ \\
\hline $\begin{array}{l}\text { Random resource } \\
\text { capacities }\end{array}$ & \multicolumn{2}{|c|}{4} & \multicolumn{2}{|c|}{6} & \multicolumn{2}{|c|}{8} & \multicolumn{2}{|c|}{10} \\
\hline
\end{tabular}

When the previously formulated IP \& CP models of the crisp equivalent lower, middle and upper level problems are run under these fuzzy \& stochastic data through ILOG CPLEX optimization studio version 12.10 on an Intel Core i7 $2.4 \mathrm{GHz}$ IBM PC, optimization results are obtained as given in Table 5. The fuzzy expected objective values, i.e., project makespan provided by both IP and CP-based transformation approaches are also demonstrated in Fig. 4 .

Table 5 Optimization details for IP \& CP models of the crisp equivalent lower, middle and upper problems

\begin{tabular}{lcccccc}
\hline \multirow{1}{*}{ Optimization results } & \multicolumn{3}{c}{ IP-based transformation } & \multicolumn{2}{c}{ CP-based transformation } \\
\cline { 2 - 7 } & $\begin{array}{c}\text { Lower } \\
\text { Level }\end{array}$ & $\begin{array}{c}\text { Middle } \\
\text { Level }\end{array}$ & $\begin{array}{c}\text { Upper } \\
\text { Level }\end{array}$ & $\begin{array}{c}\text { Lower } \\
\text { Level }\end{array}$ & $\begin{array}{c}\text { Middle } \\
\text { Level }\end{array}$ & $\begin{array}{c}\text { Upper } \\
\text { Level }\end{array}$ \\
\hline Model class & & PILP & & & CP & \\
Total integer variables & 3106 & 4098 & 5090 & 272 & 272 & 272 \\
Total constraints & 722 & 577 & 770 & 2169 & 276 & 2169 \\
Total non-zero elements & 21186 & 28113 & 41474 & $*$ & $*$ & $*$ \\
Total solver iterations & 21 & 27 & - & $*$ & $*$ & $*$ \\
Solutions found & 4 & 3 & 1 & 1 & 7 & 1 \\
Optimality gap (\%) & - & - & - & - & - & - \\
CPU time (sec.) & 0.03 & 0.20 & 0.19 & 0.07 & 6.62 & 0.95 \\
\hline Fuzzy expected makespan & \multicolumn{7}{c}{ (5.812, 8.4375, 11.0625) } & & $(5.875,8.4375,11.0)$ \\
\hline
\end{tabular}




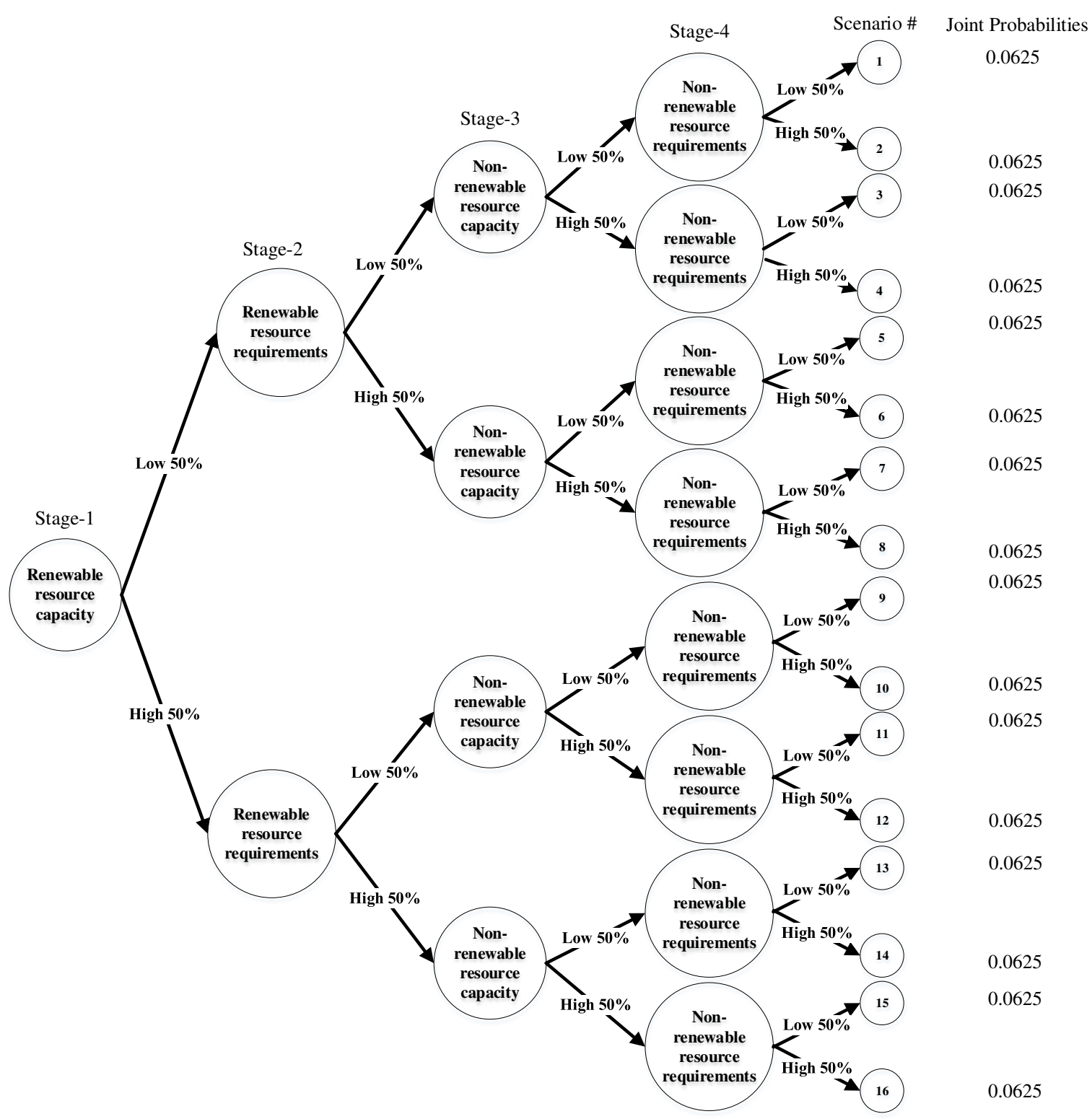

Fig. 3 Scenario tree for stochastic capacities of the resources and resource requirements of the project activities

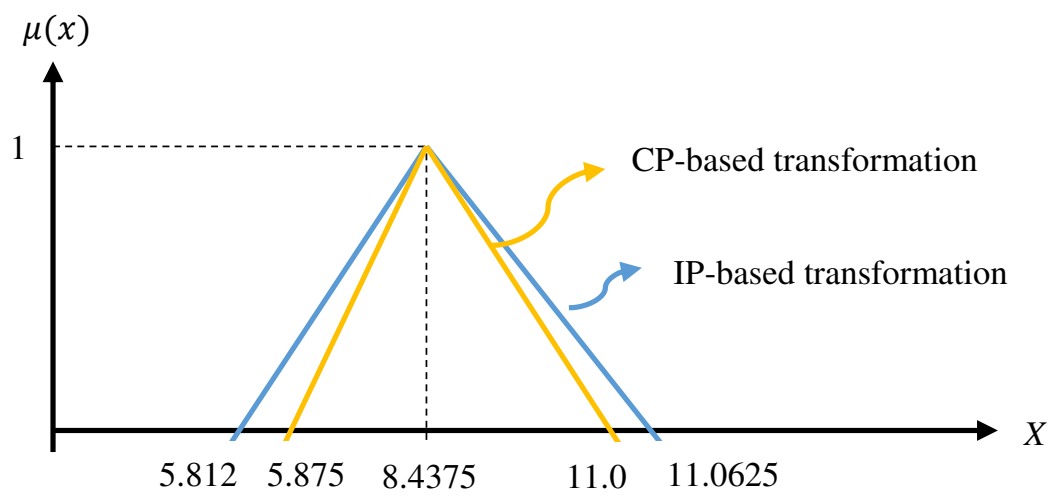

Fig. 4 Comparison of membership functions for fuzzy expected project makespan by IP \& CP-based approaches

When the results of the IP and CP-based transformation approaches are compared, it is recognized that they found the same most likely or core value (8.4375) for the expected project makespan objective. This means that the IP and CP models of the middle level problem 
generated the same objective value. However, the left and right margins of the fuzzy expected project makespan objective are differentiated as clearly seen in Fig. 4. This is because of different alternative optimal solutions may found by solving the middle level problem via IP and CP-based transformation approaches. In detail, solution of the middle level problem by IP and CP-based approaches leads to different alternative solutions with the same objective value, i.e., 8.4375. Although these alternative optimal solutions have the same objective value, they include different mode selections for some of the project activities. However, as mentioned previously in Sections 4.1 and 4.2, any given project activity should be executed under the same mode for all of the middle, lower and upper level problems. In other words, selected modes by solving the lower and upper level problems have to be the same with the chosen modes by solution of the middle level problem. For this reason, alternative optimal solutions of the middle level problem with different mode selections may result different left and right margins for the fuzzy expected project makespan. Fortunately, when we compared these fuzzy expected project makespan values, i.e., $(5.812,8.4375,11.0625)$ and $(5.875,8.4375,11.0)$ by using any fuzzy ranking technique, it is clear that none of them is superior or inferior to the other. In other words, both of these fuzzy expected project makespan values can be equally accepted by the project manager(s). For this reason, it can be concluded that there is no significant differences between fuzzy efficient solutions found by the IP and CP-based transformation approaches. Furthermore, these fuzzy efficient solutions are also found within similar computational times. Details of fuzzy completion times of the project activities obtained from the proposed IP and CP-based transformation approaches are also presented in Appendix A. It is obviously seen in this appendix that except scenario\#7, the same execution modes are selected under all of the scenarios and the same fuzzy project makespan are obtained. However, different modes, i.e., 1 versus 2 , are selected by the proposed IP and CP-based approaches for activity\#3 under scenario\#7. Therefore, different fuzzy project makespan values, i.e., $(7,11,15)$ and $(8,11,14)$ are generated for scenario\#7. Actually, this difference in scenario\#7 causes aforementioned variation in the fuzzy expected project makespan values, i.e., $(5.812,8.4375,11.0625)$ and $(5.875,8.4375,11.0)$. Lastly, some of the generated fuzzy project schedules under different scenarios can be visualized by the following Gantt charts as in Figs. $5-8$.
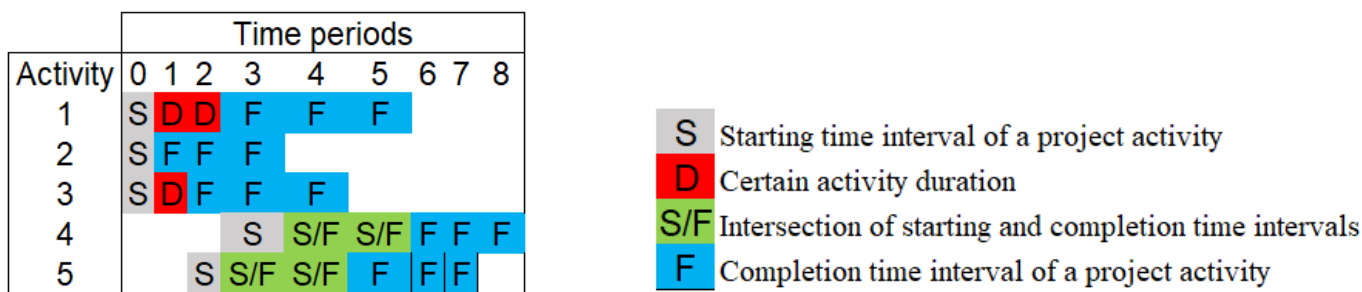

Fig. 5 Gantt chart of a fuzzy project schedule under high resource capacities \& low requirements (scenario\#11)

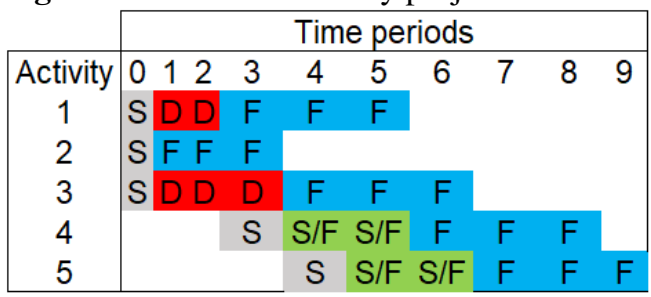

Fig. 6 Gantt chart of a fuzzy project schedule under low resource capacities \& low requirements (scenario\#1)

\begin{tabular}{|c|c|c|c|c|c|c|c|c|}
\hline & \multicolumn{8}{|c|}{ Time periods } \\
\hline Activity & 012 & 4 & 5 & 6 & 7 & 8 & 9 & 1011 \\
\hline 1 & $S D D F$ & $\mathrm{~F}$ & $\mathrm{~F}$ & & & & & \\
\hline 2 & $\mathrm{~s}$ & $S \mathrm{~S} / \mathrm{F}$ & $\mathrm{S} / \mathrm{F}$ & $\mathrm{F}$ & $\mathrm{F}$ & $\mathrm{F}$ & & \\
\hline 3 & SDD & $\mathrm{DF}$ & $\mathrm{F}$ & $\mathrm{F}$ & & & & \\
\hline 4 & $\mathrm{~s}$ & $S$ S/F & $\mathrm{S} / \mathrm{F}$ & $S / F$ & & $\mathrm{~F}$ & $\mathrm{~F}$ & \\
\hline 5 & & S & S/F & $S / F$ & & & & $\mathrm{~F}$ \\
\hline
\end{tabular}

Fig. 7 Gantt chart of a fuzzy project schedule under high resource capacities \& high requirements (scenario\#16) 


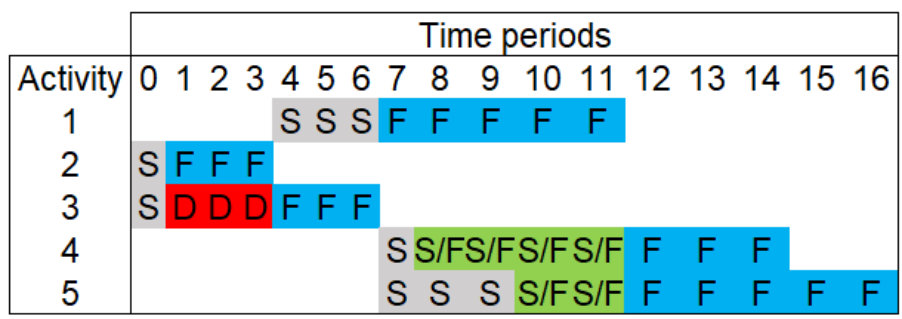

Fig. 8 Gantt chart of a fuzzy project schedule under low resource capacities \& high requirements (scenario\#6)

It is clearly seen in Fig. $5-8$ that project makespan will be influenced by the stochastic resources' capacities and the amounts of resource requirements of the project activities. In fact, fuzzy expected project makespan will be longer in case of the lower resource capacities and the higher resource requirements of the project activities (see in Fig. 8 and scenario\#6). Some of the corresponding renewable resource profiles for the generated solutions under different scenarios\#6 and \#11 are also demonstrated in the following Figs. $9-10$.
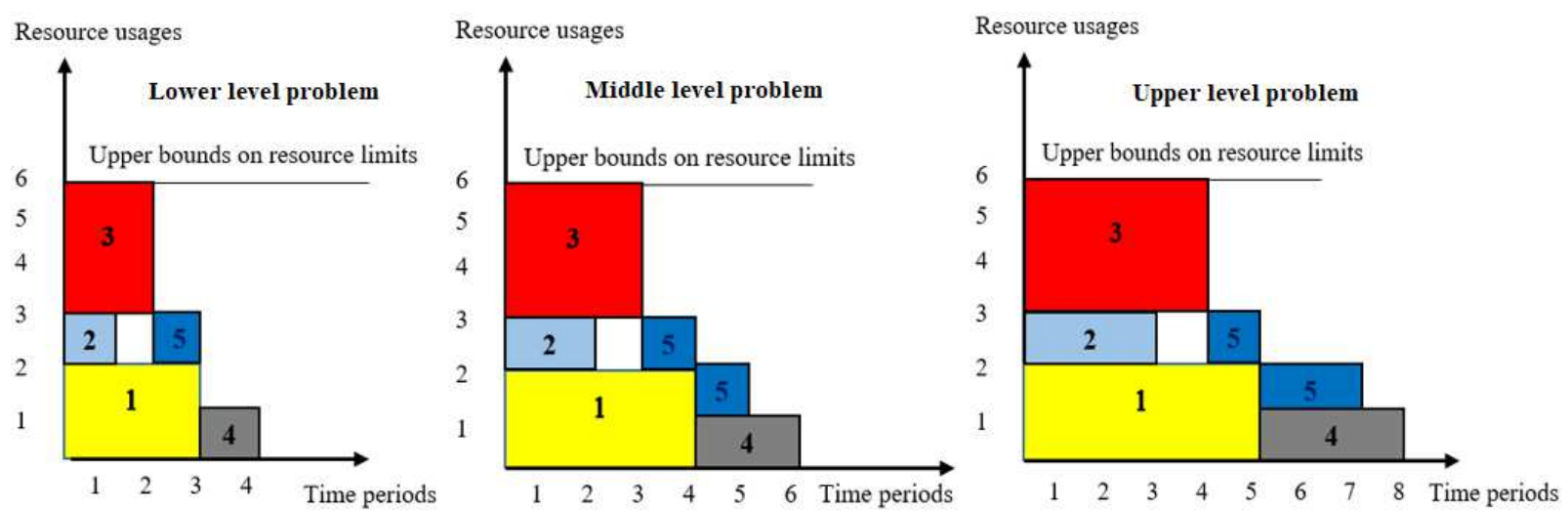

Fig. 9 The renewable resource profiles under high capacity \& low requirements (scenario\#11).

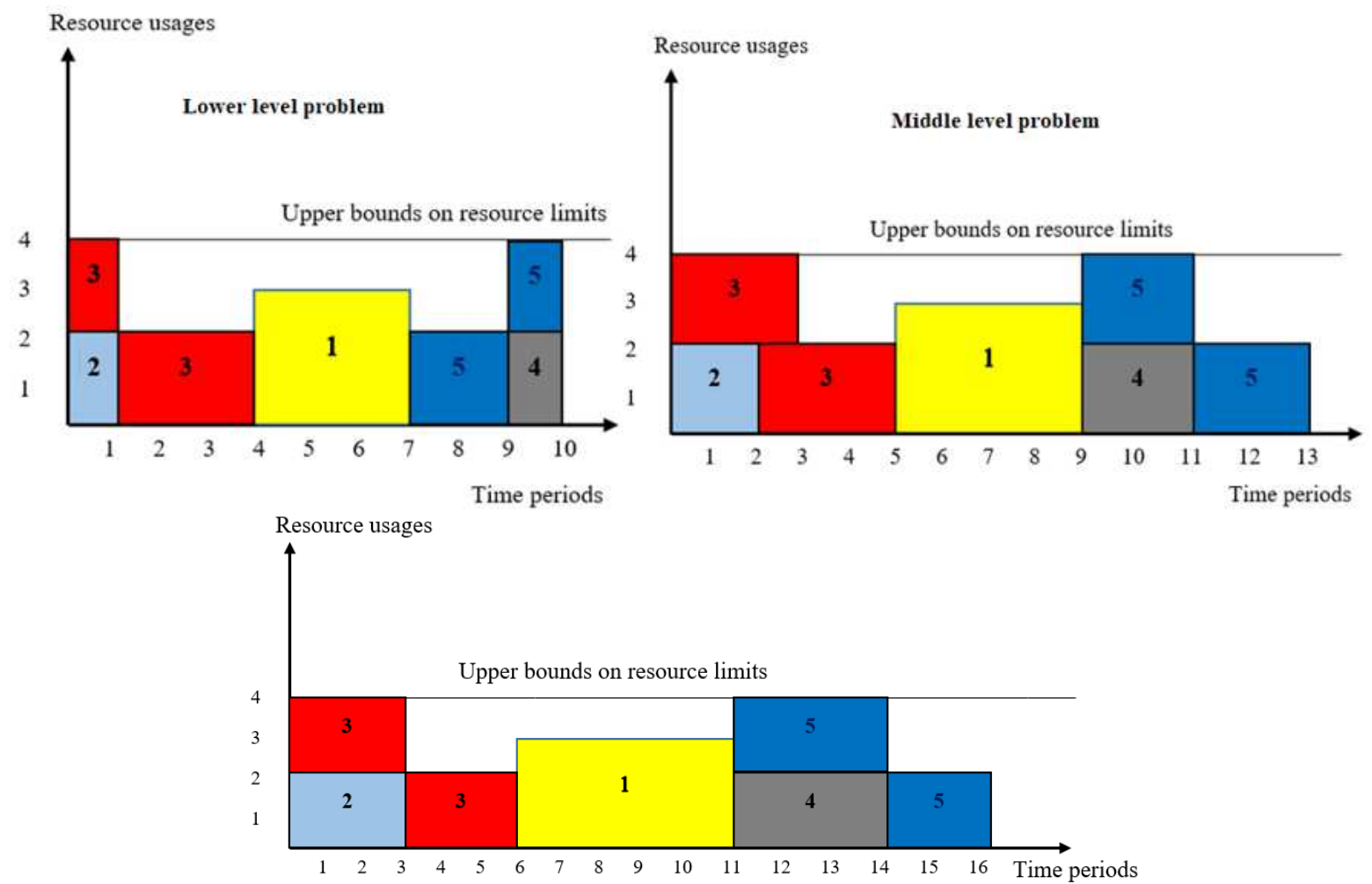

Fig. 10 Renewable resource profiles under low capacity \& high requirements (scenario\#6) 


\subsection{Weighted additive fuzzy goal program (WAFGP) for the multi-objective FS-MRIPSP}

In order to cope with the conflicting objectives simultaneously (i.e., minimizing the project makespan and total resource costs) and produce compromise fuzzy project schedules to the project manager(s), a weighted additive fuzzy goal programming method is applied. This method integrates weighted membership functions of fuzzy goals whose relative importance degrees or weights may have different values. Indeed, a single weighted linear utility function is utilized by this method to express the overall preference of the decision maker(s) for aggregating the relative importance of these fuzzy goals. Actually, this utility function is computed by multiplying the membership function of each fuzzy goal with their importance weights. Then, the provided results are added together to provide maximal satisfaction of the fuzzy goals (Amid et al., 2009; Baykasoğlu \& Subulan, 2016). In this paper, we also modified this classical WAFGP method by associating it with the previously mentioned bound \& decomposition principle in Sections 4.1 and 4.2. Because, traditional WAFGP method is just able to generate crisp compromise solutions under uncertainty of the aspiration levels of the multiple objectives. However, we desire to obtain fuzzy efficient solutions (or fuzzy project schedules) which trade-off between the project makespan and total resource usage costs. To do this, fuzzy payoff matrix should be first constructed after solving the previously formulated ILP or CP models of the single-objective middle, lower and upper level problems independently. Firstly, ILP or CP model of the middle level problem should be solved for each goal separately. Subsequently, bound constraints should be added to the ILP or CP models of the lower and upper level problems for each objective according to the solution of middle level problem. Then, these lower and upper level problems should also be solved for each objective individually. Thereafter, a fuzzy payoff matrix as shown in Table 6 can be obtained from singleobjective optimization results of the middle, lower and upper level problems.

Table 6 The corresponding fuzzy payoff matrix

\begin{tabular}{ccc}
\hline Objectives & $\mathrm{Z} 1$ & $\mathrm{Z} 2$ \\
\hline $\mathrm{Z} 1$ & $\left(Z 1_{l}^{*}, Z 1_{m}^{*}, Z 1_{u}^{*}\right)$ & $\left(Z 2_{l}, Z 2_{m}, Z 2_{u}\right)$ \\
$\mathrm{Z} 2$ & $\left(Z 1_{l}, Z 1_{m}, Z 1_{u}\right)$ & $\left(Z 2_{l}^{*}, Z 2_{m}^{*}, Z 2_{u}^{*}\right)$ \\
\hline
\end{tabular}

Fuzzy values in the main diagonal of this matrix, i.e., $\left(Z 1_{l}^{*}, Z 1_{m}^{*}, Z 1_{u}^{*}\right)$ and $\left(Z 2_{l}^{*}, Z 2_{m}^{*}, Z 2_{u}^{*}\right)$, denote the individual best solutions of the middle, lower and upper level problems for each goal. Thus, these values can be used as benchmarks to determine the ideal/reference points. In addition, the remaining values within this matrix, i.e., $\left(Z 1_{l}, Z 1_{m}, Z 1_{u}\right)$ and $\left(Z 2_{l}, Z 2_{m}, Z 2_{u}\right)$ can be used as lower-upper bounds on the fuzzy goals. Bu using this fuzzy payoff matrix, aspiration level and max-min limits of each objective function can be determined under uncertainty. On the other hand, project managers can also decide these reference values, lower and upper limits of the goals based on their experiences and preferences. Fortunately, determination of these values by using such a fuzzy payoff matrix guarantees feasibility of each goal in the solution space. After generating this fuzzy payoff matrix, membership function of each fuzzy goal should be constructed by using these reference values and max-min limits. In detail, membership function values represent the satisfaction levels of the goals (Baykasoğlu \& Subulan, 2016). For instance, membership functions of these fuzzy goals are constructed as in Eqs. (84) - (85) for the middle level problem and they are also demonstrated in Fig. 11.

$$
\begin{aligned}
\mu_{1}^{m} & \leq \frac{Z 2_{m}-Z \text { Comp }}{Z \text { makespan }} \\
\mu_{2}^{m}-Z 2_{m}^{*} & \leq \frac{Z 1_{m}-Z \text { Comp } p_{\text {cost }}^{m}}{Z 1_{m}-Z 2_{m}^{*}}
\end{aligned}
$$



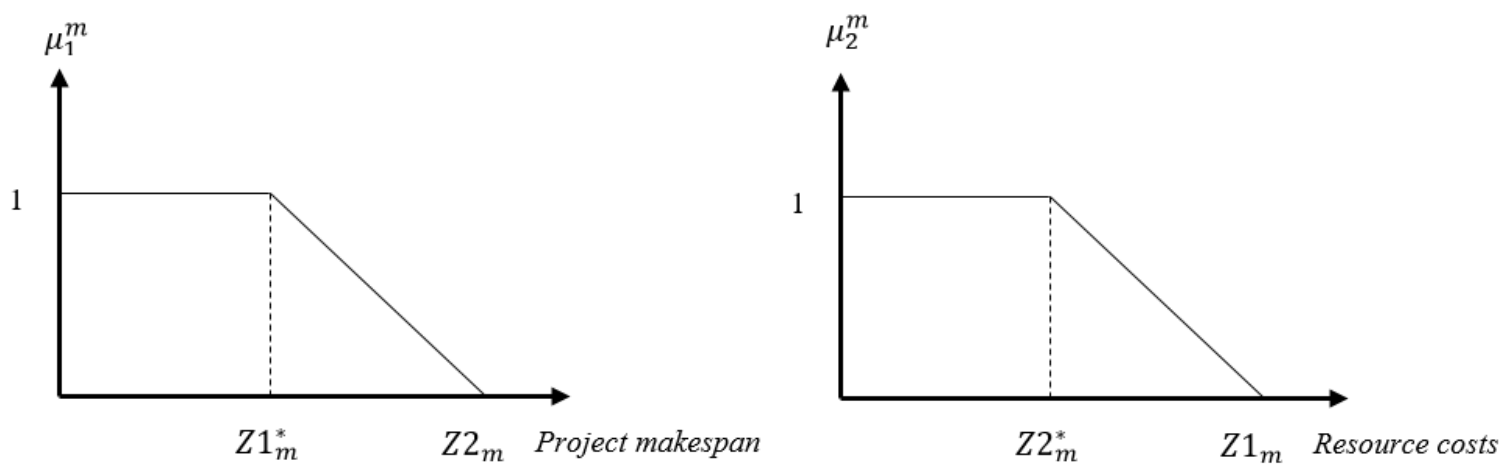

Fig. 11 Membership functions of fuzzy goals in the middle level problem: a) Project makespan, b) Resource costs

In Eqs. (95) - (96), ZComp makespan and ZComp cost represent the core or most likely values of fuzzy goals in the compromise solution that will be generated by the WAFGP method. Afterwards, the relative importance weights of these objectives, i.e., $w_{1}$ and $w_{2}$ should be specified according to their priorities in such a way that higher weights are assigned to the objectives with higher priority levels. Lastly, ILP and CP models of the crisp equivalent middle level problem can be reformulated as in Eqs. (97) - (98) for the multi-objective FS-MRIPSP.

Maximize $w_{1} \cdot \mu_{1}^{m}+w_{2} \cdot \mu_{2}^{m}$

Subject to;

Constraint sets related to the membership functions given in Eqs. (95) - (96).

Constraint sets in Eqs. (23) - (30) for the ILP model or Eqs. (64) - (70) for the CP model.

$0 \leq \mu_{1}^{m}, \mu_{2}^{m} \leq 1$

In Eq. (97), it is intended to maximize summation of weighted satisfaction degrees of the fuzzy goals. The lower/upper bounds on these satisfaction levels or membership function values are also taken into consideration in Eq. (98). After solving the above WAFGP model for the middle level problem, bounds on the fuzzy-stochastic decision variables (or project schedules and resources' availability levels) and the objective values can be easily provided to reformulate the ILP or CP models of the lower and upper level problems. By using the WAFGP method, ILP and CP models of crisp equivalent upper level problem are modified as in Eqs. (99) - (109).

Maximize $w_{1} \cdot \mu_{1}^{u}+w_{2} \cdot \mu_{2}^{u}$

Subject to;

$\mu_{1}^{u} \leq \frac{Z 2_{u}-Z \operatorname{Comp} p_{\text {makespan }}^{u}}{Z 2_{u}-Z 1_{u}^{*}}$

$\mu_{2}^{u} \leq \frac{Z 1_{u}-Z \operatorname{Comp} p_{\text {cost }}^{u}}{Z 1_{u}-Z 2_{u}^{*}}$

Constraint sets in Eqs. (31) - (32), (35) - (38) and (42) - (43) for the ILP model.

Constraint sets in Eqs. (71) - (72), and (76) - (79) for the CP model.

ZComp makespan $\geq Z$ Comp makespan

$Z \operatorname{Comp} p_{\text {cost }}^{u} \geq Z \operatorname{Comp} p_{\text {cost }}^{m}$

$t_{u} \cdot x_{j q t_{u} s} \geq t(\mathrm{comp})_{m}^{*} \cdot x(\mathrm{comp})_{j q^{*} t_{m}^{*} s} \quad \forall j \in J, \forall s \in S, q=q(\mathrm{comp})^{*}$ for the ILP model (104)

alternative $\left(X_{j s u},\left\{Y_{j q s u} \mid q=q(\mathrm{comp})^{*}\right\}\right) \quad \forall j \in J, \forall s \in S \quad$ for the CP model

endOf $\left(Y_{j q s u} \mid q=q(\mathrm{comp})^{*}\right) \geq \operatorname{endO} f\left(Y(\mathrm{comp})_{j q^{*} s m}\right) \forall j \in J, \forall s \in S$ for the CP model (106)

$K R_{r s u} \geq K R(\mathrm{comp})_{r s m}^{*} \quad \forall r \in R, \forall s \in S \quad$ for both ILP \& CP models

$K N_{n s u} \geq K N(\mathrm{comp})_{n s m}^{*} \quad \forall n \in N, \forall s \in S \quad$ for both ILP \& CP models

$0 \leq \mu_{1}^{u}, \mu_{2}^{u} \leq 1$

Membership functions of fuzzy goals, i.e., $\mu_{1}^{u}$ and $\mu_{2}^{u}$ are first constructed in Eqs. (100) (101) for the upper level problem. In the above formulation, it should be emphasized here that 
Eqs. (104) should be used instead of Eqs. (105) - (106) when the ILP model of the upper level problem is solved. In the opposite case, Eq. (104) should be removed and Eqs. (105) - (106) should be actively used while solving the CP model. Eqs. (107) -(108) should be added to both of the ILP \& CP models of the multi-objective upper level problem for the availability levels of the project resources. As mentioned previously, Eqs. (102) - (103) ensure that the right margins or pessimistic values of the fuzzy objectives in the produced compromise solution have to be greater than their core or most likely values provided by the solution of middle level problem. Eqs. (104) - (106) guarantee that the same execution modes chosen in the middle level problem should be selected again in the upper level problem. Moreover, the right margins (or pessimistic values) of the fuzzy completion times of the activities have to be greater than their core or most likely values in the compromise solutions. In a similar way, ILP and CP models of the crisp equivalent lower level problem can be revised as in Eqs. (110) - (121).

Maximize $w_{1} \cdot \mu_{1}^{l}+w_{2} \cdot \mu_{2}^{l}$

Subject to;

$\mu_{1}^{l} \leq \frac{Z 2_{l}-Z C_{\text {omp }}^{l} l \text { makespan }}{Z 2_{l}-Z 1_{l}^{*}}$

$\mu_{2}^{l} \leq \frac{Z 1_{l}-Z \operatorname{Comp} p_{\text {cost }}^{l}}{Z 1_{l}-Z 2_{l}^{*}}$

Constraint sets in Eqs. (44) - (45), (48) - (51) and (56) - (57) for the ILP model.

Constraint sets in Eqs. (83) - (84) and (89) - (91) for the CP model.

ZComp makespan $_{\text {ma }}^{l}$ ZComp makespan

$Z \operatorname{Comp} p_{\text {cost }}^{l} \leq Z \operatorname{Comp} p_{\text {cost }}^{m}$

$t_{l} \cdot x_{j q t_{l} s} \leq t(\mathrm{comp})_{m}^{*} \cdot x(\mathrm{comp})_{j q^{*} t_{m}^{*} s} \quad \forall j \in J, \forall s \in S, q=q(\mathrm{comp})^{*}$ for the ILP model

$\sum_{q \neq q(\text { comp })^{*}}^{Q_{j}} \sum_{t_{l}=E F T_{j l}}^{L F T_{j l}} t_{l} \cdot x_{j q t_{l} s}=0 \quad \forall j \in J, \forall s \in S$ for the ILP model

alternative $\left(X_{j s l},\left\{Y_{j q s l} \mid q=q(\mathrm{comp})^{*}\right\}\right) \quad \forall j \in J, \forall s \in S \quad$ for the CP model

endOf $\left(Y_{j q s l} \mid q=q(\mathrm{comp})^{*}\right) \leq \operatorname{endOf}\left(Y(\mathrm{comp})_{j q^{*} \mathrm{sm}}\right) \forall j \in J, \forall s \in S$ for the CP model

$K R_{r s l} \leq K R(\text { comp })_{r s m}^{*}$ $\forall r \in R, \forall s \in S \quad$ for both ILP \& CP models

$K N_{n s l} \leq K N(\mathrm{comp})_{n s m}^{*}$ $\forall n \in N, \forall s \in S \quad$ for both ILP \& CP models

$0 \leq \mu_{1}^{l}, \mu_{2}^{l} \leq 1$

In Eqs. (111) - (112), membership functions of the lower level problem, i.e., $\mu_{1}^{l}$ and $\mu_{2}^{l}$ are constructed based on the fuzzy payoff matrix given in Table 6 . Then, the original constraints of the ILP or CP models are incorporated into the WAFGP. According to the Eqs. (113) - (114), the left margins or optimistic values of fuzzy objectives in the produced compromise solutions have to be less than their core or most likely values. If the ILP model is used, Eqs. (115) - (116) should be included instead of Eqs. (117) - (118). Eqs. (115) - (118) maintain that the same execution modes chosen in the middle level problem should also be selected again in the lower level problem and the left margins (or optimistic values) of the fuzzy completion times of the activities have to be less than their core or most likely values in the compromise solutions. After solving ILP or CP models of the middle, lower and upper level problems using the modified $W A F G P$ method, several fuzzy compromise project schedules can be derived. Summary of the proposed ILP \& CP-based transformation approaches with a bound \& decomposition principle can be depicted by a flow chart to gain a better understanding. As clearly seen in Fig. 12, the proposed transformation approach can be realized on both ILP and CP models and is composed of two subsequent solution phases, namely single and multi-objective optimization. 


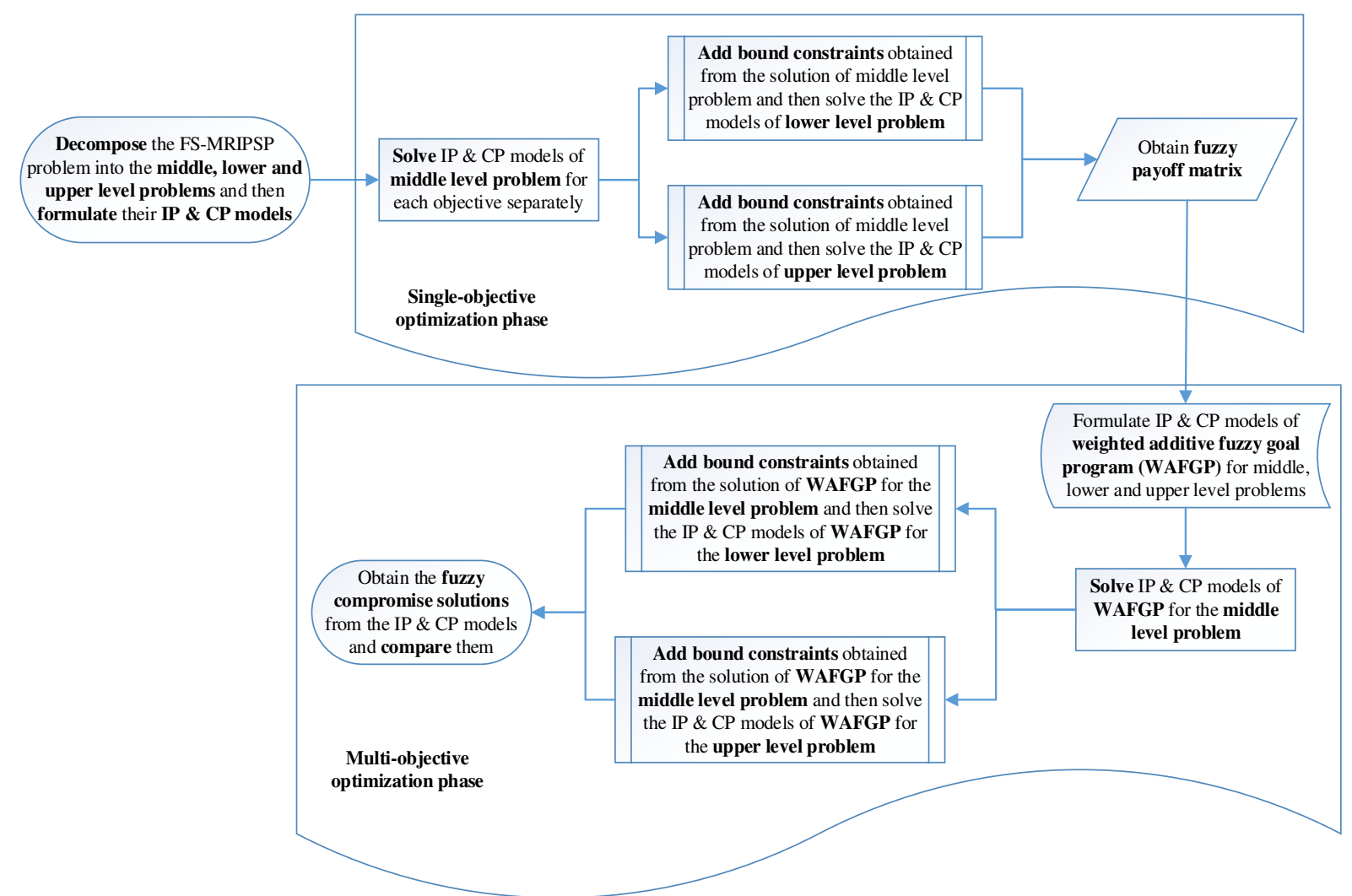

Fig. 12 Flowchart of the bound \& decomposition based solution methodology for multi-objective FS-MRIPSP

\section{A case study of POM module group project implementation of an ERP software}

\subsection{Company introduction and data description}

In order to verify the proposed transformation approach and display its validity and applicability, an application study is presented for a production-and-operations management (POM) module implementation process of an international ERP software company in Turkey. There are over 200 professional ERP consultants, several R\&D centers and solution partners of this company in Turkey, Germany, India, United Arab Emirates, South Korea, Switzerland and United States. It offers many services and innovative ERP solutions in 16 languages in 32 different countries with more than 1000 customers and more than 30.000 simultaneous active users. Actually, it provides industry-specific solutions in many kinds of sectors like automotive, aviation-defense, printing, packaging, textile-garment, machinery and industrial automation etc. It also supports comprehensive services including software sales \& development, consultancy, maintenance, education, project management. This company has also many quality certificates like ISO/IEC 27001 information security management, ISO/IEC15504 software process improvement \& capability determination (SPICE) and ISO 9001:2015 (Subulan, 2020). Fig. 13 displays its sales, R\&D and consulting network all over the world. After sales of its ERP software product, project implementation, planning and scheduling problems will arise and therefore, it is needed to configure customized solutions with respect to the customers' own business process, requirements and expectations. To do this, it is required to prepare a detailed project plan and activity schedules by creating the work-breakdownstructure (WBS) and setting different project phases. To gain a better understanding, common steps for the project implementation of this ERP Company are summarized in Fig. 14. In this research, we mainly focused on the POM module group project implementation of this software company in one of its medium-sized customer firm. 


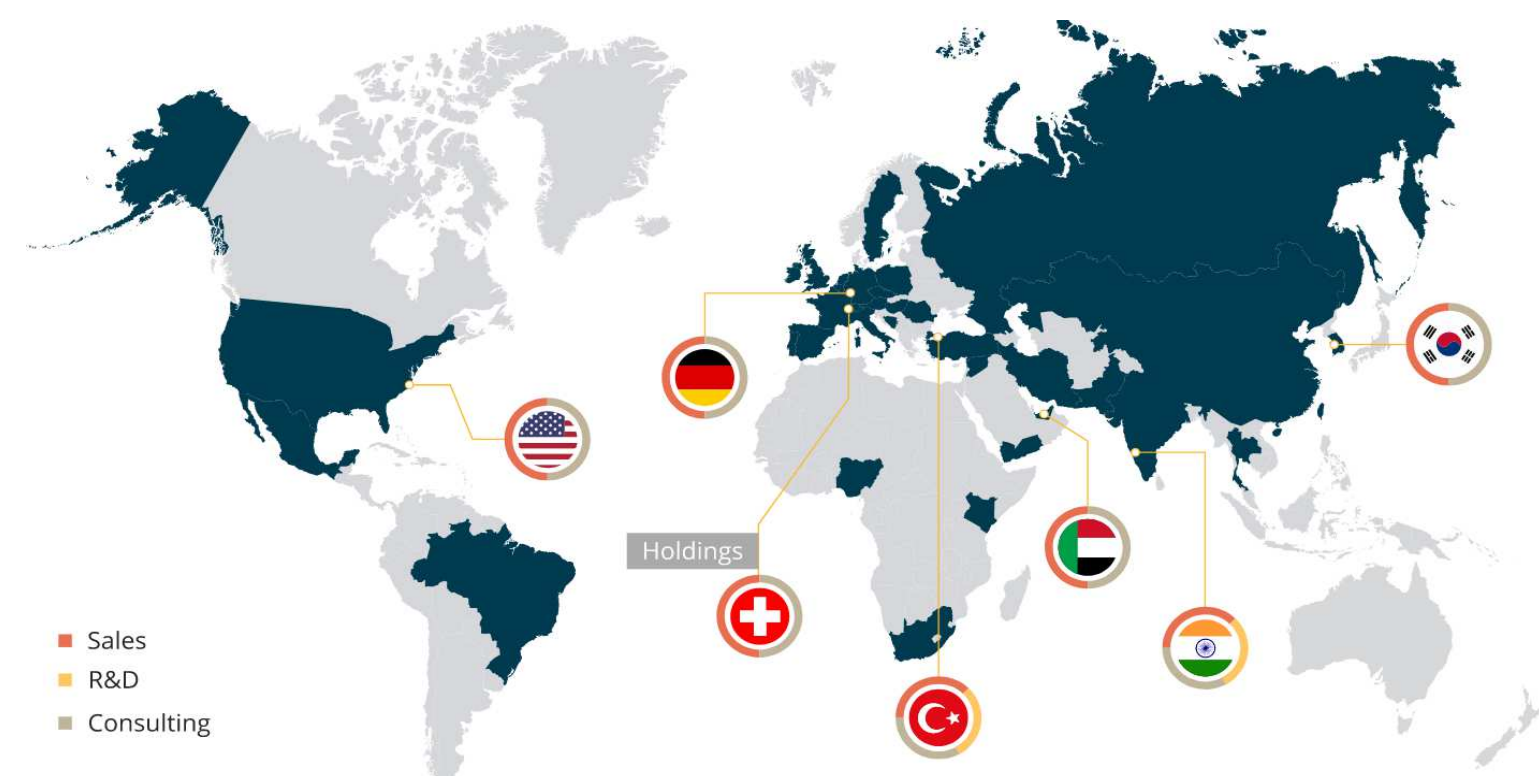

Fig. 13 Sales, R\&D and consulting network of the ERP Company all over the world (IAS Inc., 2021)

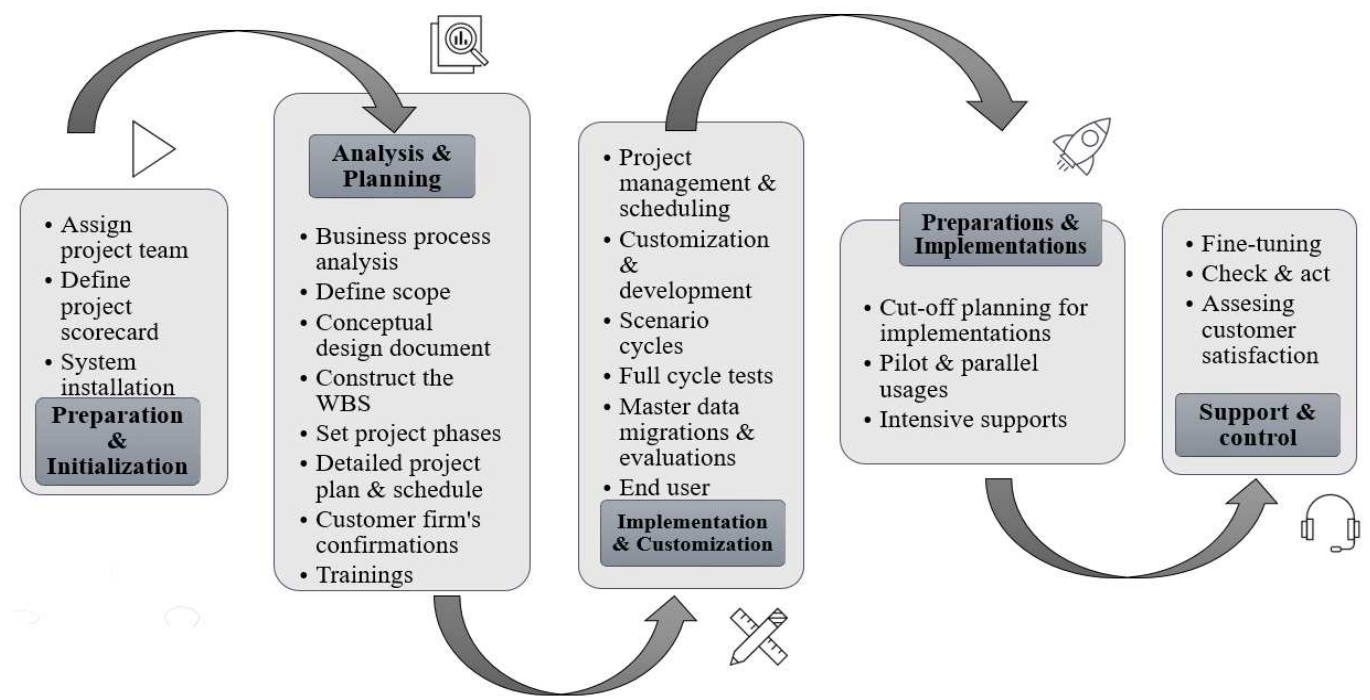

Fig. 14 Common steps of the project implementation methodology of the ERP Company (IAS Inc., 2021)

Actually, project implementation plan with a detailed schedule is generally required for the POM module integration process of this ERP software after preparing the sales agreement with each customer firm. Indeed, ERP product of this software company has modular structure and its POM module group is composed of several modules such as basic core data and system implementation (BAS), bill-of-materials (BOM) management, production routing (ROU), inventory management and control (INV), production planning and control (PRD), material requirements planning (MRP), quality management - statistical process control (QLT) and finally capacity planning and production scheduling (CAP). Except these POM modules, there are also several other modules like system management (SYS), sales management (SAL), purchasing management (PUR), billing verification and control (VER), project management (PRJ), customer relationships management (CRM), standard cost calculation (CAL), general and financial accounting (FIN), cost center accounting - production costing (COS), Budget management (BUD), human resources management (HCM), collaborator management (COL), business process management (BPM) etc. Recently, a comprehensive study was also performed by Subulan (2020) for integration process of all of these ERP modules under mixed interval \& stochastic types of uncertainties. However, the present research has just concentrated on the 
POM module group project implementation of this ERP software product so as to elaborate main project activities related to the module integration processes. To do this, totally 44activities are first described as given in Table 7 for implementation process of each module in the POM group. Thereafter, precedence relations among these project activities and the integration processes of different modules are specified by the ERP consultants and the activityon-node (AON) project network is drawn as in Fig. 15. As listed in Table 7, these POM modules have some common project activities associated with designing and adaptation of support tables, data collection, analysis of the business processes of the customer firm, designing some output reports and documents for each POM module etc. Actually, these general project activities should be performed for each module in a similar way and therefore, they can be stated as the milestones of the module integration process in the ERP project implementation. On the other hand, there are also module-specific project activities which can be realized for only a pre-specified system module. For instance, project activity 6.3 which is named as "Defining quality plans: quality characteristics, sampling methods, center lines, specification limits, defect classes with their priorities" is only associated with the process quality control (QLT) module. As mentioned previously, these project activities in the module integration processes may interact with each other and therefore, the precedence relations and interactions between these modules should be taken into account while producing the project schedules. For example, BOM and production routing (ROU) modules have to be already integrated into the customer firm's business system before installation and implementation of material requirement planning (MRP) and production planning (PRD) modules. In a similar manner, before integrating the capacity planning and production scheduling (CAP) module, all of the INV, MRP and PRD modules should be integrated earlier as clearly shown in Fig. 15. The immediate predecessors of each project activity has already presented in Table 7. As shown in this table, we also defined multiple execution modes for each project activity since the activity durations may be differentiated with respect to the amounts of allocated project resources. By the way, it should be emphasized here that human resources are defined as the renewable project resources throughout this case study. Particularly, different types of human resources like ERP software company's consultants and process experts in the customer firm are considered as the renewable project resources. Because, professional human resources and knowledge/expertise of the consultants are the primary sources of an ERP software company and play a critical role in successful ERP project implementation (Motwani et al., 2002; Tadinen, 2005; Plaza, 2016; Subulan, 2020). Moreover, experienced process experts in the customer firm are as vital as the success of ERP consultants. Because, these experts support the ERP consultants for improving the firm's business processes and developing innovative ERP solutions.

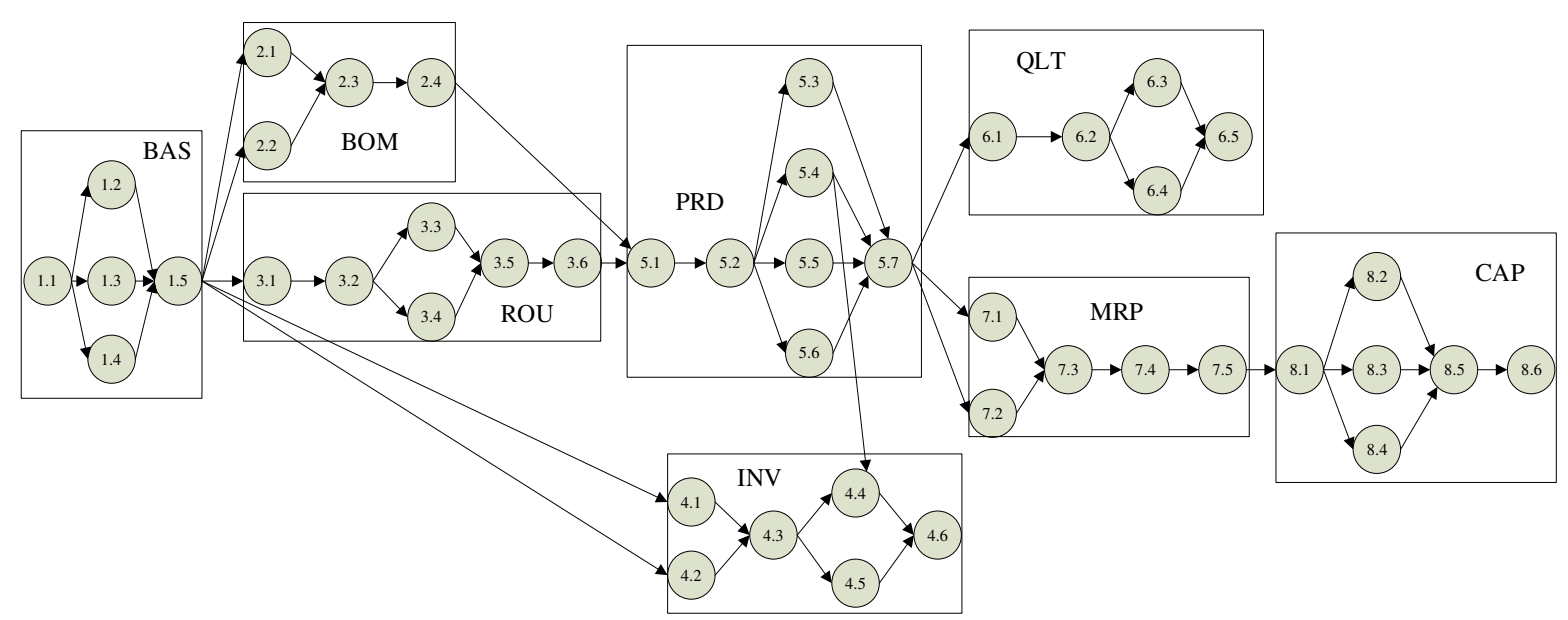

Fig. 15 The AON project network diagram for POM module group implementation process of the ERP software 
Table 7 Fuzzy data for the production and operations management (POM) module group implementation project of the ERP system

\begin{tabular}{|c|c|c|c|c|c|c|}
\hline \multirow{2}{*}{$\begin{array}{l}\text { Implementation Process of the POM Module Group } \\
\text { (Activity Descriptions) }\end{array}$} & \multirow{2}{*}{$\begin{array}{c}\text { Immediate } \\
\text { Predecessors }\end{array}$} & \multicolumn{3}{|c|}{ Fuzzy Duration (Day) } & \multirow{2}{*}{$\begin{array}{c}\text { Fuzzy Earliest } \\
\text { Finish Time (EFT) }\end{array}$} & \multirow{2}{*}{$\begin{array}{l}\text { Fuzzy Latest Finish } \\
\text { Time (LFT) }\end{array}$} \\
\hline & & Mode-1 & Mode-2 & Mode-3 & & \\
\hline \multicolumn{7}{|l|}{ 1. BAS (Basic Core Data) Module } \\
\hline 1.1 Define material types, codes and records & - & $(1,2,3)$ & $(2,3,4)$ & $(4,5,6)$ & $(1,2,3)$ & $(102,134,163)$ \\
\hline 1.2 Specify material supply categories & 1.1 & $(0,1,1)$ & $(1,2,2)$ & $(2,3,4)$ & $(1,3,4)$ & $(104,137,167)$ \\
\hline 1.3 Fictionalize the variant structures & 1.1 & $(0,1,2)$ & $(1,2,3)$ & - & $(1,3,5)$ & $(104,137,167)$ \\
\hline 1.4 Determine relationships between materials and storage system & 1.1 & $(2,3,4)$ & $(2,4,5)$ & $(3,5,6)$ & $(3,5,7)$ & $(104,137,167)$ \\
\hline 1.5 Transfer of the basic core data & $1.2,1.3,1.4$ & $(0,1,2)$ & $(1,2,3)$ & $(3,4,5)$ & $(3,6,9)$ & $(104,138,169)$ \\
\hline \multicolumn{7}{|l|}{ 2. BOM (Bill-of-Material) Module } \\
\hline 2.1. Analyze BOM structures and define alternative BOMs & 1.5 & $(2,3,4)$ & $(3,4,5)$ & $(4,5,6)$ & $(5,9,13)$ & $(112,150,182)$ \\
\hline 2.2 Design and adapt support tables for BOM \& analyze variant structures & 1.5 & $(3,4,5)$ & $(4,6,6)$ & $(5,6,7)$ & $(6,10,14)$ & $(112,150,182)$ \\
\hline 2.3 Specify quantities and stock keeping units (SKUs) & $2.1,2.2$ & $(1,2,2)$ & $(2,3,4)$ & - & $(7,12,16)$ & $(113,152,184)$ \\
\hline 2.4 Transfer of the BOM data via electronic data interchange (EDI) & 2.3 & $(1,2,3)$ & $(2,3,5)$ & $(3,4,5)$ & $(8,14,19)$ & $(114,154,187)$ \\
\hline \multicolumn{7}{|l|}{ 3. ROU - Production Routing Module } \\
\hline 3.1 Analyze manufacturing processes \& evaluate production routing structures & 1.5 & $(4,5,6)$ & $(6,7,7)$ & $(7,8,10)$ & $(7,11,15)$ & $(108,143,175)$ \\
\hline 3.2 Specify types of operations and routing alternatives & 3.1 & $(1,2,2)$ & $(1,2,3)$ & - & $(8,13,17)$ & $(109,145,177)$ \\
\hline 3.3 Design and adapt support tables for ROU module & 3.2 & $(4,5,6)$ & - & - & $(12,18,23)$ & $(113,150,183)$ \\
\hline 3.4 Generate variant dependent operation codes & 3.2 & $(1,2,3)$ & $(2,3,3)$ & $(2,4,5)$ & $(9,15,20)$ & $(113,150,183)$ \\
\hline 3.5 Data transfer for the operations, work-centers and cost-centers & $3.3,3.4$ & $(1,3,3)$ & $(2,3,4)$ & $(3,4,4)$ & $(13,21,26)$ & $(114,153,186)$ \\
\hline 3.6 Provide material network diagrams (Operation process charts) & 3.5 & $(0,1,1)$ & $(1,2,2)$ & $(1,2,3)$ & $(13,22,27)$ & $(114,154,187)$ \\
\hline \multicolumn{7}{|l|}{ 4. INV - Inventory Management Module } \\
\hline 4.1 Analyze inventory movements and prepare stock documents & 1.5 & $(2,3,4)$ & $(2,4,5)$ & $(3,5,5)$ & $(5,9,13)$ & $(137,186,228)$ \\
\hline 4.2 Determine lot-sizing policies and analyze the barcode system & 1.5 & $(3,4,5)$ & $(3,5,6)$ & $(5,6,7)$ & $(6,10,14)$ & $(137,186,228)$ \\
\hline 4.3 Design and adapt support tables for INV module & $4.1,4.2$ & $(4,5,6)$ & $(5,6,7)$ & $(7,8,9)$ & $(10,15,20)$ & $(141,191,234)$ \\
\hline 4.4 Integrate sales, procurement, production and inventory modules & $4.3,5.4$ & $(6,8,9)$ & $(7,8,10)$ & - & $(26,41,51)$ & $(147,199,243)$ \\
\hline 4.5 Specify costing methods and perform stock valuation for accounting & 4.3 & $(1,2,3)$ & - & - & $(11,17,23)$ & $(147,199,243)$ \\
\hline 4.6 Integrate activity-based costing, accounting and inventory modules & $4.4,4.5$ & $(3,4,5)$ & $(4,5,6)$ & - & $(29,45,56)$ & $(150,203,248)$ \\
\hline \multicolumn{7}{|l|}{ 5. PRD - Production Planning \& Control Module } \\
\hline 5.1 Analyze production process, collect manufacturing and process data & $2.4,3.6$ & $(3,5,6)$ & $(4,6,7)$ & $(5,7,8)$ & $(16,27,33)$ & $(117,159,193)$ \\
\hline 5.2 Design and adapt support tables for production planning module & 5.1 & $(4,5,7)$ & - & - & $(20,32,40)$ & $(121,164,200)$ \\
\hline 5.3 Fictionalize the production planning \& work order types and structures & 5.2 & $(0,1,1)$ & $(1,2,2)$ & $(1,2,3)$ & $(20,33,41)$ & $(122,166,202)$ \\
\hline 5.4 Analyze the relationships between work orders \& inventory movements & 5.2 & $(0,1,2)$ & $(1,2,3)$ & $(2,3,4)$ & $(20,33,42)$ & $(122,166,202)$ \\
\hline 5.5 Define shift schedules and associate it with the company's work calendar & 5.2 & $(0,1,1)$ & $(0,1,2)$ & $(1,2,2)$ & $(20,33,41)$ & $(122,166,202)$ \\
\hline 5.6 Analyze contract manufacturing operations and residual products & 5.2 & $(1,2,2)$ & $(1,2,3)$ & $(3,4,4)$ & $(21,34,42)$ & $(122,166,202)$ \\
\hline 5.7 Design output reports and documents for production planning \& control & $5.3,5.4,5.5,5.6$ & $(3,4,5)$ & $(5,6,7)$ & - & $(24,38,47)$ & $(125,170,207)$ \\
\hline
\end{tabular}


Table 7 Fuzzy project data for the production and operations management (POM) module group of the ERP system (Continued)

\begin{tabular}{|c|c|c|c|c|c|c|}
\hline \multirow{2}{*}{$\begin{array}{l}\text { Implementation Process of the POM Module Group } \\
\text { (Activity Descriptions) }\end{array}$} & \multirow{2}{*}{$\begin{array}{l}\text { Immediate } \\
\text { Predecessors }\end{array}$} & \multicolumn{3}{|c|}{ Fuzzy Duration (Day) } & \multirow{2}{*}{$\begin{array}{c}\text { Fuzzy Earliest } \\
\text { Finish Time (EFT) }\end{array}$} & \multirow{2}{*}{$\begin{array}{l}\text { Fuzzy Latest Finish } \\
\text { Time (LFT) }\end{array}$} \\
\hline & & Mode-1 & Mode-2 & Mode-3 & & \\
\hline \multicolumn{7}{|l|}{ 6. QLT - Process Quality Control Module } \\
\hline 6.1 Analyze the operational quality control process of the company & 5.7 & $(1,2,2)$ & $(2,3,4)$ & - & $(25,40,49)$ & $(144,194,237)$ \\
\hline 6.2 Design and adapt support tables for process quality control module & 6.1 & $(3,4,5)$ & $(3,5,6)$ & $(4,6,7)$ & $(28,44,54)$ & $(147,198,242)$ \\
\hline 6.3 Define quality plans: quality characteristics, sampling methods, center & 6.2 & $(2,3,4)$ & $(3,4,5)$ & $(4,5,6)$ & $(30,47,58)$ & $(149,201,246)$ \\
\hline \multicolumn{7}{|l|}{ lines, specification limits, defect classes with their priorities } \\
\hline 6.4 Data collection from the control/test operations in manufacturing & 6.2 & $(1,2,3)$ & $(2,3,4)$ & $(3,4,5)$ & $(29,46,57)$ & $(149,201,246)$ \\
\hline 6.5 Design output reports: quality control charts \& process capability analysis & $6.3,6.4$ & $(1,2,2)$ & $(2,3,4)$ & - & $(31,49,60)$ & $(150,203,248)$ \\
\hline \multicolumn{7}{|l|}{ 7. MRP - Materials Requirement Planning Module } \\
\hline $\begin{array}{l}\text { 7.1 Determine basic MRP parameters: Timing, procedures, order } \\
\text { quantity/production lot-sizing methods for production and procurement etc. }\end{array}$ & 5.7 & $(2,3,3)$ & $(2,3,4)$ & $(3,4,5)$ & $(26,41,50)$ & $(127,173,210)$ \\
\hline 7.2 Specify MRP scenario types and structures & 5.7 & $(0,1,1)$ & $(1,2,3)$ & - & $(24,39,48)$ & $(127,173,210)$ \\
\hline 7.3 Design and adapt support tables for MRP module & $7.1,7.2$ & $(3,4,5)$ & $(4,5,6)$ & $(5,6,7)$ & $(29,45,55)$ & $(130,177,215)$ \\
\hline 7.4 Implementation tests for validation and verification of the MRP outputs & 7.3 & $(2,3,3)$ & $(3,4,4)$ & $(3,4,5)$ & $(31,48,58)$ & $(132,180,218)$ \\
\hline $\begin{array}{l}7.5 \text { Design output reports which is able to identify relationships between } \\
\text { customers' sales orders \& alternative MRP solutions }\end{array}$ & 7.4 & $(2,3,5)$ & $(3,4,6)$ & $(3,5,6)$ & $(33,51,63)$ & $(134,183,223)$ \\
\hline \multicolumn{7}{|l|}{ 8. CAP - Capacity planning \& Production Scheduling Module } \\
\hline 8.1 Analyze the long-short term production capacities \& production lot-sizing & 7.5 & $(4,5,7)$ & $(5,6,8)$ & $(6,8,9)$ & $(37,56,70)$ & $(138,188,230)$ \\
\hline 8.2 Design and adapt support tables for CAP module & 8.1 & $(3,4,5)$ & - & - & $(40,60,75)$ & $(141,192,235)$ \\
\hline 8.3 Define capacity groups \& dispatching rules for scheduling & 8.1 & $(0,1,1)$ & $(1,2,3)$ & $(2,3,4)$ & $(37,57,71)$ & $(141,192,235)$ \\
\hline 8.4 Specify capacity types $\&$ resource (labor, machinery equipment) planning & 8.1 & $(1,2,3)$ & $(2,3,4)$ & $(3,4,5)$ & $(38,58,73)$ & $(141,192,235)$ \\
\hline $\begin{array}{l}\text { 8.5 Develop heuristic algorithms via an integrated development environment } \\
\text { embedded in the ERP software for scheduling work orders }\end{array}$ & $8.2,8.3,8.4$ & $(7,8,9)$ & $(9,10,12)$ & $(10,12,15)$ & $(47,68,84)$ & $(148,200,244)$ \\
\hline $\begin{array}{l}\text { 8.6 Design output reports and documents: resource utilization charts for labor } \\
\text { and machinery equipment and Gantt charts for the work order operations }\end{array}$ & 8.5 & $(2,3,4)$ & $(3,5,6)$ & - & $(49,71,88)$ & $(150,203,248)$ \\
\hline
\end{tabular}


For that reason, ERP consultants should be in cooperation and collaboration with the customer firm's process experts. Therefore, effective and efficient utilization of human resources may have significant impact on the success of ERP projects. On the other hand, these professionals have different abilities, tasks and responsibilities during the project realization.

As clearly seen in Table 7, activity durations (in days) are represented by triangular fuzzy numbers since they are estimated by the ERP consultants based on their knowledge and expertise. Indeed, optimistic, most likely and pessimistic values of the activity durations are determined by the project team consisting of ERP consultants and process experts. Unfortunately, it is a challenging task to identify deterministic values for these durations due to the highly changing and dynamic project environments. Because, activity durations may change according to industrial scale, sector and size of the customer firm. Moreover, customer firm's information system infrastructure and the development level of the ERP software in terms of its information technology level and decision support potential may also have significant effects on these durations. Furthermore, dynamic situations such as the availability/reliability of the human resources, unanticipated or extra expectations of the customer firm restrict the project managers to define deterministic activity durations. Briefly, fuzzy activity durations under different execution modes are defined as in Table 7 to cope with these uncertain conditions. Thus, the earliest and latest finish times of the project activities are computed as fuzzy numbers and given in Table 7. Actually, their values are determined by using the forward and backward pass calculations. Before applying the proposed ILP and CP-based transformation approaches, these fuzzy earliest and latest finishing times should be computed for each project activity. In a nutshell, all of the fuzzy data are presented in Table 7 for the POM module group implementation project of the ERP system. On contrary to the fuzzy activity durations, human resource requirements which are described in terms of the labor force (in man-days) and the implementation costs $(\$)$ of the project activities are defined as stochastic project parameters with pre-specified discrete probabilities. Because, ERP project managers announced that these parameters may have randomness in nature. According to the customer firm's unforeseen expectations or its additional business process requirements, human resources demanded by the project activities may change in random fashion. Moreover, efficiency and reliability factors of the human resources may influence the standard durations and resource requirements of the project activities. Actually, effective working hours and human resource utilizations may be affected by these factors. As highlighted by Subulan (2020), inexperienced ERP consultants may cause inefficient operations/processes and therefore, the longer activity durations and the higher human resource requirements may arise. Additionally, the day offs, sick leaves and shirking may result in unreliable human resources. Furthermore, since the ERP consultants are responsible for different projects of several customer firms, their effective and efficient working hours in a specific firm may decrease due to the over workload. This may cause decreases in both efficiency and reliability of the human resources. As a result, utilization rates of the human resources may drop and the project makespan gets longer. For all of these reasons, human resources requirements of the project activities are described as discrete random variables under different execution modes and presented in Table 8. To do this, different scenario probabilities are defined by the ERP project team for the pessimistic, most likely and optimistic values of these human resource requirements. As obviously seen in Table 8, the higher amounts of human resources are demanded in case of shorter activity durations. On the other hand, the less amount of human resources are needed under some modes whose activity durations get longer. It should also be noted here that probabilistic human resource requirements are described for the process experts by considering the standardized ERP solutions and implementation-oriented innovative ERP solutions. Because, standardized ERP solutions may not be suitable for some of the customer firms' business processes and therefore, additional implementations and adaptations may be required for more customized ERP solutions. 
Table 8 Stochastic human resource requirements of the POM module group implementation project's activities under different execution modes

\begin{tabular}{|c|c|c|c|c|c|c|c|c|c|c|c|c|c|c|c|}
\hline \multirow{3}{*}{$\begin{array}{c}\text { Project } \\
\text { Activities }\end{array}$} & \multicolumn{9}{|c|}{ ERP Supplier's Consultants (in man-days) } & \multicolumn{6}{|c|}{ Process Experts in Customer (in man-days) } \\
\hline & \multicolumn{3}{|c|}{$\begin{array}{c}\text { Optimistic } \\
\text { (Scenario probability } 30 \%) \\
\end{array}$} & \multicolumn{3}{|c|}{$\begin{array}{c}\text { Most Likely } \\
\text { (Scenario probability } 40 \% \text { ) }\end{array}$} & \multicolumn{3}{|c|}{$\begin{array}{c}\text { Pessimistic } \\
\text { (Scenario probability } 30 \%)\end{array}$} & \multicolumn{3}{|c|}{$\begin{array}{l}\text { Standardized ERP solutions } \\
\text { (Scenario probability } 40 \%)\end{array}$} & \multicolumn{3}{|c|}{$\begin{array}{l}\text { (Implementation oriented) } \\
\text { (Scenario probability 60\%) }\end{array}$} \\
\hline & Mode-1 & Mode-2 & Mode-3 & Mode-1 & Mode-2 & Mode-3 & Mode-1 & Mode-2 & Mode-3 & Mode-1 & Mode-2 & Mode-3 & Mode-1 & Mode-2 & Mode-3 \\
\hline 1.1 & 12 & 8 & 4 & 15 & 9 & 6 & 20 & 15 & 12 & 3 & 2 & 1 & 5 & 3 & 2 \\
\hline 1.2 & 8 & 5 & 3 & 10 & 7 & 5 & 15 & 12 & 10 & 2 & 1 & 0 & 3 & 2 & 1 \\
\hline 1.3 & 4 & 3 & - & 6 & 4 & - & 10 & 8 & - & 1 & 0 & - & 2 & 1 & - \\
\hline 1.4 & 15 & 12 & 9 & 18 & 15 & 10 & 22 & 18 & 13 & 4 & 2 & 1 & 6 & 5 & 3 \\
\hline 1.5 & 18 & 14 & 10 & 20 & 16 & 12 & 25 & 20 & 15 & 5 & 3 & 2 & 7 & 5 & 4 \\
\hline 2.1 & 16 & 15 & 12 & 20 & 18 & 15 & 24 & 20 & 18 & 6 & 4 & 2 & 8 & 6 & 4 \\
\hline 2.2 & 20 & 18 & 15 & 25 & 22 & 18 & 30 & 26 & 22 & 1 & 0 & 0 & 2 & 1 & 0 \\
\hline 2.3 & 9 & 7 & - & 12 & 10 & - & 16 & 13 & - & 4 & 3 & - & 4 & 3 & - \\
\hline 2.4 & 6 & 4 & 2 & 8 & 5 & 3 & 10 & 7 & 5 & 1 & 0 & 0 & 2 & 1 & 0 \\
\hline 3.1 & 25 & 18 & 15 & 30 & 21 & 18 & 36 & 27 & 24 & 10 & 7 & 6 & 28 & 25 & 20 \\
\hline 3.2 & 10 & 7 & - & 12 & 8 & - & 18 & 12 & - & 8 & 5 & - & 10 & 7 & - \\
\hline 3.3 & 20 & - & - & 24 & - & - & 30 & - & - & 5 & - & - & 8 & - & - \\
\hline 3.4 & 13 & 10 & 8 & 15 & 12 & 9 & 20 & 16 & 13 & 7 & 4 & 3 & 10 & 7 & 5 \\
\hline 3.5 & 10 & 8 & 5 & 12 & 9 & 6 & 15 & 12 & 10 & 6 & 3 & 2 & 8 & 6 & 4 \\
\hline 3.6 & 2 & 1 & 0 & 3 & 2 & 1 & 5 & 4 & 2 & 1 & 0 & 0 & 1 & 0 & 0 \\
\hline 4.1 & 20 & 18 & 15 & 25 & 22 & 18 & 32 & 26 & 23 & 6 & 5 & 4 & 12 & 10 & 8 \\
\hline 4.2 & 17 & 15 & 12 & 20 & 18 & 16 & 25 & 22 & 20 & 7 & 6 & 4 & 15 & 10 & 8 \\
\hline 4.3 & 24 & 20 & 17 & 30 & 25 & 20 & 34 & 29 & 25 & 4 & 3 & 2 & 8 & 6 & 5 \\
\hline 4.4 & 33 & 30 & - & 40 & 35 & - & 45 & 40 & - & 5 & 2 & - & 10 & 7 & - \\
\hline 4.5 & 12 & - & - & 15 & - & - & 20 & - & - & 4 & - & - & 10 & - & - \\
\hline 4.6 & 16 & 12 & - & 20 & 16 & - & 25 & 20 & - & 4 & 3 & - & 7 & 5 & - \\
\hline 5.1 & 32 & 28 & 25 & 40 & 35 & 30 & 45 & 40 & 35 & 10 & 8 & 6 & 30 & 25 & 20 \\
\hline 5.2 & 18 & - & - & 21 & - & - & 26 & - & - & 5 & - & - & 8 & - & - \\
\hline 5.3 & 6 & 4 & 3 & 8 & 6 & 4 & 10 & 8 & 6 & 4 & 3 & 2 & 7 & 5 & 3 \\
\hline 5.4 & 5 & 3 & 2 & 6 & 4 & 3 & 8 & 6 & 4 & 3 & 2 & 1 & 5 & 4 & 2 \\
\hline 5.5 & 2 & 1 & 0 & 3 & 2 & 1 & 5 & 4 & 2 & 1 & 0 & 0 & 2 & 1 & 0 \\
\hline 5.6 & 4 & 3 & 2 & 6 & 4 & 3 & 8 & 6 & 4 & 3 & 2 & 1 & 4 & 3 & 2 \\
\hline 5.7 & 10 & 7 & - & 14 & 10 & - & 18 & 13 & - & 4 & 2 & - & 5 & 3 & - \\
\hline
\end{tabular}


Table 8 Stochastic human resource requirements of the POM module group implementation project's activities under different execution modes (Continued)

\begin{tabular}{|c|c|c|c|c|c|c|c|c|c|c|c|c|c|c|c|}
\hline \multirow{3}{*}{$\begin{array}{c}\text { Project } \\
\text { Activities }\end{array}$} & \multicolumn{9}{|c|}{ ERP Supplier's Consultants (in man-days) } & \multicolumn{6}{|c|}{ Process Experts in Customer (in man-days) } \\
\hline & \multicolumn{3}{|c|}{$\begin{array}{c}\text { Optimistic } \\
\text { (Scenario probability 30\%) }\end{array}$} & \multicolumn{3}{|c|}{$\begin{array}{c}\text { Most Likely } \\
\text { (Scenario probability 40\%) } \\
\end{array}$} & \multicolumn{3}{|c|}{$\begin{array}{c}\text { Pessimistic } \\
\text { (Scenario probability } 30 \%) \\
\end{array}$} & \multicolumn{3}{|c|}{$\begin{array}{c}\text { Standardized ERP solutions } \\
\text { (Probability 40\%) }\end{array}$} & \multicolumn{3}{|c|}{$\begin{array}{c}\text { (Implementation oriented) } \\
\text { (Probability 60\%) }\end{array}$} \\
\hline & Mode-1 & Mode-2 & Mode-3 & Mode-1 & Mode-2 & Mode-3 & Mode-1 & Mode-2 & Mode-3 & Mode-1 & Mode-2 & Mode-3 & Mode-1 & Mode-2 & Mode-3 \\
\hline 6.1 & 10 & 6 & - & 12 & 8 & - & 15 & 10 & - & 10 & 8 & - & 12 & 9 & - \\
\hline 6.2 & 22 & 19 & 16 & 28 & 24 & 20 & 31 & 26 & 23 & 6 & 4 & 3 & 8 & 6 & 4 \\
\hline 6.3 & 20 & 17 & 12 & 24 & 20 & 16 & 27 & 23 & 20 & 13 & 12 & 10 & 18 & 16 & 13 \\
\hline 6.4 & 16 & 13 & 10 & 20 & 16 & 12 & 22 & 18 & 16 & 20 & 17 & 14 & 25 & 20 & 16 \\
\hline 6.5 & 13 & 8 & - & 16 & 10 & - & 19 & 14 & - & 4 & 3 & - & 6 & 4 & - \\
\hline 7.1 & 20 & 17 & 12 & 25 & 20 & 15 & 30 & 25 & 20 & 8 & 6 & 4 & 10 & 8 & 6 \\
\hline 7.2 & 7 & 5 & - & 9 & 6 & - & 13 & 10 & - & 4 & 2 & - & 5 & 3 & - \\
\hline 7.3 & 16 & 14 & 11 & 21 & 18 & 14 & 26 & 21 & 18 & 5 & 3 & 2 & 7 & 5 & 3 \\
\hline 7.4 & 20 & 17 & 14 & 24 & 21 & 18 & 28 & 25 & 20 & 9 & 7 & 5 & 12 & 10 & 8 \\
\hline 7.5 & 15 & 12 & 9 & 18 & 15 & 12 & 20 & 18 & 16 & 4 & 3 & 2 & 6 & 4 & 3 \\
\hline 8.1 & 25 & 22 & 19 & 30 & 28 & 25 & 36 & 30 & 28 & 15 & 12 & 8 & 20 & 16 & 11 \\
\hline 8.2 & 34 & - & - & 40 & - & - & 45 & - & - & 5 & - & - & 8 & - & - \\
\hline 8.3 & 10 & 8 & 5 & 12 & 10 & 7 & 15 & 12 & 8 & 2 & 1 & 1 & 4 & 3 & 2 \\
\hline 8.4 & 11 & 9 & 7 & 15 & 12 & 10 & 20 & 16 & 12 & 7 & 5 & 3 & 10 & 7 & 5 \\
\hline 8.5 & 40 & 36 & 32 & 50 & 45 & 40 & 60 & 50 & 45 & 0 & 0 & 0 & 0 & 0 & 0 \\
\hline 8.6 & 27 & 24 & - & 32 & 28 & - & 36 & 32 & - & 5 & 3 & - & 7 & 5 & - \\
\hline
\end{tabular}


As clearly seen in Tables $7-8$ that the same number of execution modes are not valid for all of the project activities. In other words, project activities may have different numbers of execution modes since some activity durations cannot be reduced even if the more human resources are allocated to them. Moreover, human resource requirements for the ERP consultants are relatively larger than the process experts of the customer firm. It should be emphasized here that availability levels of different types of human resources are not taken as constant deterministic parameters (or input data) since the ERP company also desires to determine their ideal values. In other words, while producing the ERP project schedules, it is also targeted to decide on the availability levels of the human resources. Unfortunately, deterministic values cannot be determined for these resource availability levels since all of the project data are stated as uncertain. In other words, it is a challenging task to specify certain values for these availability levels in case of the stochastic human resource requirements of the project activities. Thus, it is intended to determine their pessimistic, most likely and optimistic values under different scenarios. In other words, availability levels of the human resources are also specified as fuzzy-stochastic decision variables (or project outputs). For that reason, the examined project scheduling problem will become a multi-objective and multi-mode resource investment project scheduling (MRIPSP) where the total usage costs of the human resources is aimed to minimize in addition to project makespan. Because, effective and efficient utilization of the human resources in ERP project implementations is as important as minimum project makespan for both of the software company and customer firms. Thus, it is a crucial issue to handle these conflicting objectives simultaneously.

In addition to these renewable human resources, project fund or budget of the customer firm is taken into consideration as the non-renewable project resource since its availability is limited throughout the project planning horizon. Similarly, its availability level is also defined as a decision variable since total implementation cost of different POM modules is also targeted to minimize in addition to the total human resource costs. Moreover, based on the former financial indicators and accounting reports of the customer firm, availability level of the project fund cannot determined precisely due to the dynamic nature of the incoming/outgoing cash flows along with the project planning horizon. For that reason, it is also defined as a fuzzy-stochastic decision variable and expected to take fuzzy monetary values under various scenarios.

Finally, stochastic implementation costs of different POM module integration operations (or project activities) under different execution modes are presented in Table 9. In a similar manner, ERP consultants evaluated these implementation cost values under different scenarios with discrete occurrence probabilities. All of the uncertain project data presented in Tables 7-9 are determined by the ERP consultants and process experts based on their past experiences in the comparable or similar customer firms and industries. As mentioned previously, these ERP project data may change completely from one customer firm to another with respect to its industrial scale and information technology infrastructure. Based on these random data related to the human resource requirements and implementation costs of the project activities, 18different scenarios with unequal joint probabilities are derived as shown in Fig. 16.

In the next sub-section, details of the single and multi-objective optimization results of the proposed ILP \& CP-based transformation approaches are presented and compared to each other for the present case study. 
Table 9 Stochastic implementation costs (\$) with discrete probabilities (\%) under different execution modes

\begin{tabular}{|c|c|c|c|c|c|c|c|c|c|}
\hline \multirow[t]{2}{*}{ Activities } & \multicolumn{3}{|c|}{$\begin{array}{c}\text { Optimistic Scenario } \\
\text { (Probability 25\%) }\end{array}$} & \multicolumn{3}{|c|}{$\begin{array}{c}\text { Most Likely Scenario } \\
\text { (Probability 50\%) }\end{array}$} & \multicolumn{3}{|c|}{$\begin{array}{c}\text { Pessimistic Scenario } \\
\text { (Probability 25\%) }\end{array}$} \\
\hline & Mode-1 & Mode-2 & Mode-3 & Mode-1 & Mode-2 & Mode-3 & Mode-1 & Mode-2 & Mode-3 \\
\hline 1.1 & 160 & 120 & 95 & 200 & 150 & 120 & 230 & 175 & 140 \\
\hline 1.2 & 65 & 50 & 40 & 80 & 60 & 50 & 92 & 70 & 60 \\
\hline 1.3 & 55 & 40 & - & 70 & 50 & - & 81 & 60 & - \\
\hline 1.4 & 80 & 65 & 55 & 100 & 80 & 70 & 115 & 95 & 80 \\
\hline 1.5 & 160 & 130 & 95 & 200 & 160 & 120 & 230 & 185 & 140 \\
\hline 2.1 & 200 & 160 & 145 & 250 & 200 & 180 & 290 & 230 & 210 \\
\hline 2.2 & 400 & 330 & 300 & 500 & 420 & 380 & 575 & 485 & 430 \\
\hline 2.3 & 240 & 220 & - & 300 & 280 & - & 345 & 320 & - \\
\hline 2.4 & 280 & 240 & 210 & 350 & 300 & 270 & 405 & 345 & 310 \\
\hline 3.1 & 145 & 120 & 95 & 180 & 150 & 120 & 210 & 175 & 140 \\
\hline 3.2 & 65 & 60 & - & 80 & 75 & - & 95 & 85 & - \\
\hline 3.3 & 260 & - & - & 320 & - & - & 370 & - & - \\
\hline 3.4 & 90 & 75 & 65 & 110 & 95 & 80 & 125 & 110 & 95 \\
\hline 3.5 & 275 & 245 & 240 & 340 & 310 & 300 & 390 & 360 & 345 \\
\hline 3.6 & 65 & 55 & 50 & 80 & 70 & 65 & 90 & 80 & 75 \\
\hline 4.1 & 200 & 175 & 160 & 250 & 220 & 200 & 290 & 255 & 230 \\
\hline 4.2 & 145 & 135 & 120 & 180 & 165 & 150 & 210 & 190 & 175 \\
\hline 4.3 & 175 & 160 & 145 & 220 & 200 & 185 & 250 & 230 & 210 \\
\hline 4.4 & 320 & 300 & - & 400 & 380 & - & 460 & 430 & - \\
\hline 4.5 & 300 & - & - & 380 & - & - & 440 & - & - \\
\hline 4.6 & 330 & 310 & - & 420 & 390 & - & 490 & 450 & - \\
\hline 5.1 & 400 & 360 & 320 & 500 & 450 & 400 & 575 & 520 & 460 \\
\hline 5.2 & 330 & - & - & 420 & - & - & 485 & - & - \\
\hline 5.3 & 200 & 175 & 150 & 250 & 220 & 190 & 290 & 250 & 220 \\
\hline 5.4 & 145 & 120 & 95 & 180 & 150 & 120 & 210 & 170 & 140 \\
\hline 5.5 & 65 & 48 & 40 & 80 & 60 & 50 & 95 & 70 & 60 \\
\hline 5.6 & 55 & 40 & 35 & 70 & 50 & 40 & 80 & 60 & 45 \\
\hline 5.7 & 360 & 320 & - & 450 & 400 & - & 520 & 460 & - \\
\hline 6.1 & 145 & 120 & - & 180 & 150 & - & 215 & 175 & - \\
\hline 6.2 & 255 & 240 & 225 & 320 & 300 & 280 & 385 & 345 & 320 \\
\hline 6.3 & 240 & 225 & 200 & 300 & 280 & 250 & 360 & 320 & 280 \\
\hline 6.4 & 185 & 160 & 145 & 230 & 200 & 180 & 270 & 230 & 205 \\
\hline 6.5 & 240 & 225 & - & 300 & 280 & - & 360 & 320 & - \\
\hline 7.1 & 280 & 240 & 215 & 350 & 300 & 270 & 420 & 345 & 310 \\
\hline 7.2 & 80 & 65 & - & 100 & 80 & - & 120 & 90 & - \\
\hline 7.3 & 400 & 360 & 320 & 500 & 450 & 400 & 600 & 520 & 460 \\
\hline 7.4 & 200 & 175 & 160 & 250 & 220 & 200 & 300 & 255 & 230 \\
\hline 7.5 & 375 & 345 & 325 & 470 & 430 & 410 & 565 & 495 & 470 \\
\hline 8.1 & 480 & 400 & 360 & 600 & 500 & 450 & 700 & 575 & 520 \\
\hline 8.2 & 420 & - & - & 520 & - & - & 620 & - & - \\
\hline 8.3 & 120 & 95 & 80 & 150 & 120 & 100 & 180 & 140 & 115 \\
\hline 8.4 & 80 & 65 & 55 & 100 & 80 & 70 & 120 & 95 & 80 \\
\hline 8.5 & 2000 & 1600 & 1440 & 2500 & 2000 & 1800 & 3000 & 2300 & 2100 \\
\hline 8.6 & 480 & 400 & - & 600 & 500 & - & 700 & 575 & - \\
\hline
\end{tabular}




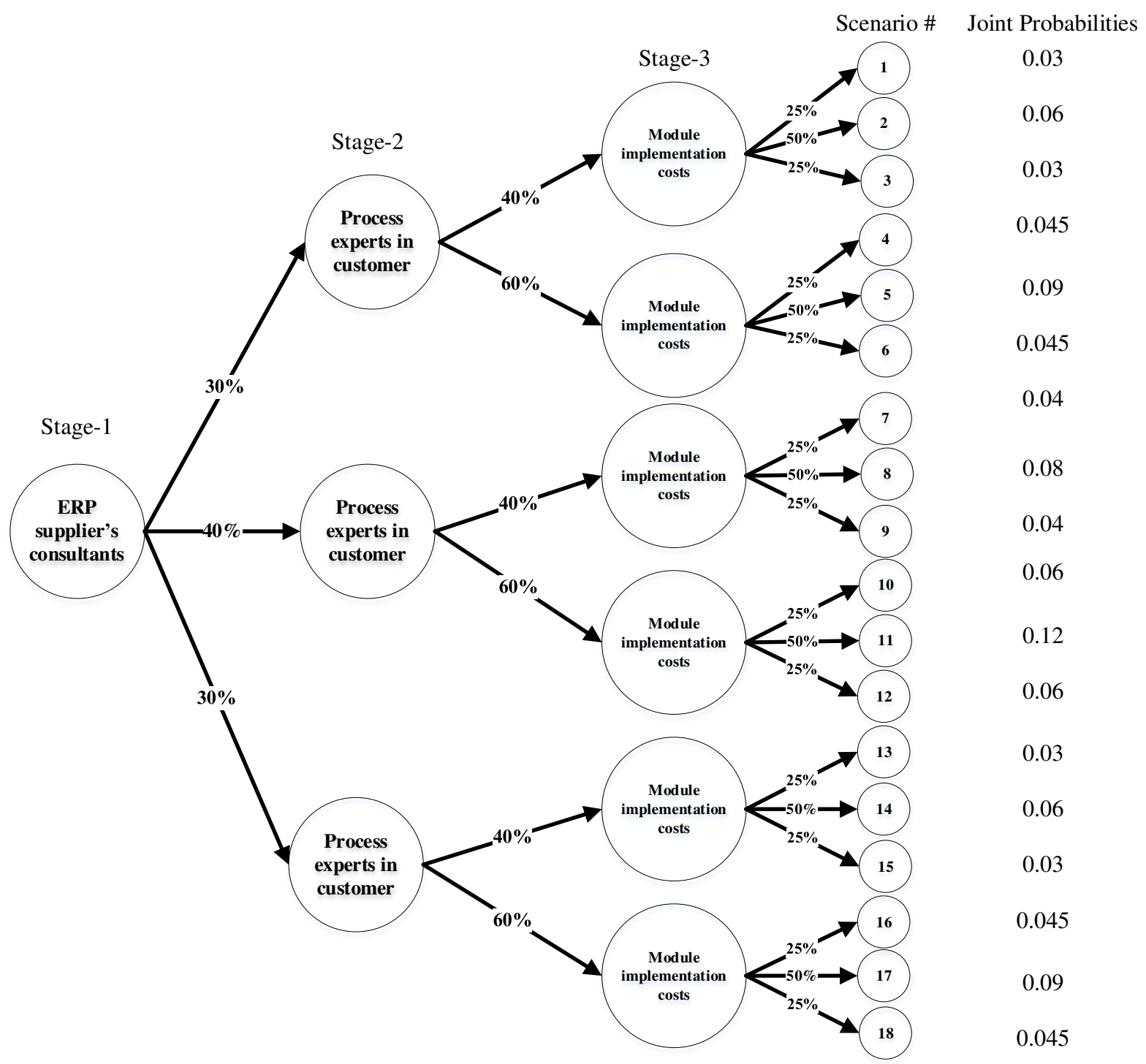

Fig. 16 Scenario tree generation for the stochastic renewable/non-renewable resource requirements

\subsection{Fuzzy-stochastic multi-objective optimization results of the proposed approaches}

In the single objective optimization phase, the middle, lower and upper level problems which were previously formulated in Sections 4.1 and 4.2 are first solved for each objective function separately by making use of an optimization suite, i.e., ILOG CPLEX optimization studio v12.10 on an Intel Corei7 $2 \mathrm{GHz}$ IBM PC. In other words, single-objective optimization results of these sub-problems are provided by both ILP \& CP-based transformation approaches. Then, the generated results are aggregated to construct the following fuzzy payoff matrices in Tables $10-11$. The details of the single-objective optimization process are also presented for each transformation approach in Tables $12-13$. As clearly seen in Tables $10-11$, the same optimal project makespan value, i.e., 71 is achieved by both ILP and CP-based transformation approaches for the middle level problem. However, since the produced solutions represent the alternative optimal solutions of this middle level problem, different objective values, i.e., 89.76 versus 92 and 49.815 versus 50 are acquired for the upper and lower level problems, respectively. Moreover, it should be noted here that optimization results of the project makespan objective are produced within reasonable computation times by both ILP and CPbased transformation approaches (see Tables $12-13$ ). 
Table 10 Fuzzy payoff matrix provided by the ILP-based transformation approach

\begin{tabular}{ccc}
\hline Objectives & Project makespan $\left(\widetilde{\boldsymbol{Z}}_{\mathbf{1}}\right)$ & Total resource costs $\left(\widetilde{\boldsymbol{Z}}_{\mathbf{2}}\right)$ \\
\hline$\widetilde{\boldsymbol{Z}}_{\mathbf{1}}$ & $(49.815,71,89.76)$ & $(20238,22011,24157)$ \\
$\widetilde{\boldsymbol{Z}}_{\mathbf{2}}$ & $(150,203,248)$ & $(13370.87,13943.8,14516.8)^{*}$ \\
\hline
\end{tabular}

Table 11 Fuzzy payoff matrix provided by the CP-based transformation approach

\begin{tabular}{ccc}
\hline Objectives & Project makespan $\left(\widetilde{\boldsymbol{Z}}_{\mathbf{1}}\right)$ & Total resource costs $\left(\widetilde{\boldsymbol{Z}}_{\mathbf{2}}\right)$ \\
\hline$\widetilde{\boldsymbol{Z}}_{\mathbf{1}}$ & $(50,71,92)$ & $(20164,21937,23710)$ \\
$\widetilde{\boldsymbol{Z}}_{\mathbf{2}}$ & $(105.25,134.66,171.53)$ & $(13523.75,14120.75,14700)^{*}$ \\
\hline
\end{tabular}

Actually, optimal value of the fuzzy expected project makespan can be achieved within relatively shorter computation times by the CP-based approach when compared to the ILPbased one. Although optimal project makespan value can be easily achieved in very shorter computation times, it is more difficult to obtain appropriate solutions for the total resource cost objective. Fortunately, optimal solution of the middle level problem with total resource cost objective is accomplished by the ILP-based approach under 2-hours runtime limit. However, solution of this middle level problem with total resource cost objective is terminated after 2hours later for the CP-based approach. In other words, CP model of the middle level problem are solved under 2-hours runtime limit and just a feasible solution with $1.26 \%$ optimality gap can be obtained. Based on these facts, it can be concluded that inclusion of the total resource cost objective which mainly uses the availability levels of the renewable and non-renewable project resources makes the solution of the stated problem much more difficult. Fortunately, it is clearly seen in Table 13 that optimal solutions for the lower and upper level problems can be easily achieved by the CP-based approach in shorter runtimes after adding the bound constraints which are obtained from the solution of middle level problem. In other words, CP-based transformation approach outperforms than the ILP-based approach in terms of the computational times for both project makespan and total resource cost objectives. According to Tables 12 - 13, although ILP-based transformation approach contains relatively large number of constraints and integer-binary variables, it leads to a better solution with a minimum fuzzy expected project makespan value of $(49.815,71,89.76)$.

Table 12 Details of single-objective optimization results obtained from the IP-based approach

\begin{tabular}{lcccccc}
\hline & \multicolumn{5}{c}{ IP-based transformation } \\
\cline { 2 - 7 } & \multicolumn{3}{c}{ Project makespan } & \multicolumn{3}{c}{ Total resource cost } \\
\cline { 2 - 7 } & Lower & Middle & Upper & Lower & Middle & Upper \\
\hline Model class & \multicolumn{2}{c}{ Pure integer linear programming (PILP) } \\
\# of constraints & 9110 & 9307 & 11810 & 9110 & 9307 & 11810 \\
\# of binary variables & 361206 & 499068 & 609012 & 361206 & 499123 & 609012 \\
\# of integer variables & 54 & 54 & 54 & 54 & 54 & 54 \\
\# of non-zero elements & 10484691 & 18155767 & 26759967 & 10484691 & 18162895 & 26759967 \\
\# of solver iterations & 0 & 674 & 2662 & 497996 & 3277467 & 83530 \\
CPU time (sec.) & 15.22 & 29.05 & 36.3 & 264.7 & 5835.6 & 100.8 \\
Optimality gap (\%) & 0 & 0 & 0 & 0 & 0 & 0 \\
\hline
\end{tabular}

Similarly, ILP-based transformation approach also outperforms than CP-based approach for the total resource cost objective in terms of the solution quality. Indeed, it provides a superior solution with a minimum fuzzy expected total resource cost value of $(13370.87,13943.8$, 14516.8). Briefly, ILP-based transformation approach leads to better quality solutions for both objective functions in the single-objective optimization process. For all of these reasons, we utilized single objective optimization results (or fuzzy payoff matrix in Table 10) of the ILPbased transformation approach to construct the membership functions in multi-objective optimization phase of the proposed solution methodology (See in Fig. 12). Again, it should also be emphasized here that CP-based transformation approach is superior in terms of the computing times in the single-objective optimization phase. 
Table 13 Details of single-objective optimization results obtained from the CP-based approach

\begin{tabular}{lcccccc}
\hline & \multicolumn{5}{c}{ CP-based transformation } \\
\cline { 2 - 7 } & \multicolumn{3}{c}{ Project makespan } & \multicolumn{3}{c}{ Total resource cost } \\
\cline { 2 - 7 } & 18766 & 2003 & 18766 & 19072 & 2022 & 19072 \\
\hline \# of constraints & 2970 & 2970 & 2970 & 2970 & 2970 & 2970 \\
\# of variables & 67907371 & 91444846 & 68907828 & 89211885 & 170872757 & 104904112 \\
Memory usage & 102 & 102 & 102 & 2960005 & 190135655 & 3431674 \\
\# of choice points & 1 & 1 & 1 & 7 & 406 & 409 \\
\# of solutions & 20106 & 3474 & 20214 & 3716931 & 271461618 & 4747263 \\
\# of branches & 72 & 72 & 72 & 750232 & 81527165 & 1042838 \\
\# of fails & 2916 & 2916 & 2916 & 2916 & 2916 & 2916 \\
\# of intervals & 0.78 & 0.57 & 1.2 & 15.5 & $7200^{*}$ & 20.12 \\
CPU time (sec.) & 0 & 0 & 0 & 0 & 1.26 & 0 \\
Optimality gap (\%) & & & & & Middle & \\
\hline
\end{tabular}

Moreover, the number of constraints and binary-integer decision variables can be significantly reduced by using such a CP-based transformation approach (see in Table 12 - 13).

In the multi-objective optimization phase, membership functions for the aspiration levels of the fuzzy objectives are first constructed by using the max-min limits given in Table 10. Then, ILP and CP models of the middle, lower and upper level problems are modified by using WAFGP method so as to obtain the compromise solutions which trade-off between project makespan and total resource cost objectives. Similar with the single-objective phase, ILP and CP models including WAFGP method are first solved for the middle level problem with different importance weights of the objectives. Unfortunately, ILP-based transformation approach cannot produce any feasible solution for some objective weights $\left(w_{1}=0.2 \& w_{2}=\right.$ $0.8 ; w_{1}=0.4 \& w_{2}=0.6$ and $w_{1}=0.6 \& w_{2}=0.4$ ) within 2-hours runtime limit. It should be highlighted here that any feasible solution cannot be generated for these objective weights even for 12-hours runtime limit. Actually, ILP-based t approach can find a feasible solution when the importance weight of the project makespan objective is increased up to $80 \%$ and the weight of the total resource cost objective decreased to $20 \%$ only $\left(w_{1}=0.8 \& w_{2}=0.2\right)$. Fortunately, several feasible solutions can be achieved by the $\mathrm{CP}$-based approach for different objective weights within just 10-minutes runtime limit without applying any search strategy (see in Table 14). In order to improve performance of the CP-based approach, we also applied some search strategies in the literature for solution of the middle level problem (Young et al., 2017; Baykasoğlu et al., 2020; IBM, 2017).

IBM ILOG CPLEX code of these search strategies are also presented in Appendix B. As shown in Table 14, significant improvements on the objective function values, i.e., Z1 and Z2 can be achieved by making use of these search strategies. On the other hand, it should be noted here that different search strategies may produce promising results for different objective weights. For instance, search strategies-2, 10, 11 and 12 generate better results for the objective weights of $w_{1}=0.2 \& w_{2}=0.8$ and $w_{1}=0.6 \& w_{2}=0.4$ whereas the best solutions are obtained by the search strategies-1, 14 and 15 for the objective weight of $w_{1}=0.4 \& w_{2}=$ 0.6. Additionally, the best objective function values can be accomplished by the search strategy-12 for the objective weight of $w_{1}=0.8 \& w_{2}=0.2$. While selecting the best search strategies to apply, we took into account the objective function with the highest weight. For example, only the values of project makespan, i.e., $\mathrm{Z} 1$ were considered when choosing the best search strategies for its highest objective weights $\left(w_{1}=0.6\right.$ and $\left.w_{1}=0.8\right)$.

After determining the best search strategies for different objective weights, the middle level problem was resolved with these weights under 2-hours runtime limit. Then, the produced solutions are used to formulate bound constraints in the lower and upper level problems. 
Table 14 Comparative study for testing different search strategies on the middle level problem of the CP-based transformation approach in the multi-objective optimization phase

\begin{tabular}{|c|c|c|c|c|c|c|c|c|c|c|c|c|c|c|c|c|c|c|}
\hline & \multicolumn{2}{|c|}{ No strategy } & \multicolumn{2}{|c|}{ Search Strategy-1 } & \multicolumn{2}{|c|}{ Search Strategy-2 } & \multicolumn{2}{|c|}{ Search Strategy-3 } & \multicolumn{2}{|c|}{ Search Strategy-4 } & \multicolumn{2}{|c|}{ Search Strategy-5 } & \multicolumn{2}{|c|}{ Search Strategy-6 } & \multicolumn{2}{|c|}{ Search Strategy-7 } & \multicolumn{2}{|c|}{ Search Strategy-8 } \\
\hline Weights & $\mathrm{Z} 1$ & $\mathrm{Z} 2$ & $\mathrm{Z} 1$ & $\mathrm{Z} 2$ & $\mathrm{Z} 1$ & $\mathrm{Z} 2$ & $\mathrm{Z} 1$ & $\mathrm{Z} 2$ & $\mathrm{Z} 1$ & $\mathrm{Z} 2$ & $\mathrm{Z} 1$ & $\mathrm{Z} 2$ & $\mathrm{Z} 1$ & $\mathrm{Z} 2$ & $\mathrm{Z} 1$ & $\mathrm{Z} 2$ & $\mathrm{Z} 1$ & $\mathrm{Z} 2$ \\
\hline $20-80 \%$ & 104.08 & 16935 & 99.96 & 16703 & 99.41 & 16914 & 99.96 & 16703 & 99.41 & 16914 & 99.96 & 16703 & 99.41 & 16914 & 99.96 & 16703 & 99.41 & 16914 \\
\hline $40-60 \%$ & 95.42 & 17184 & 92.92 & 17181 & 96.43 & 17061 & 92.92 & 17181 & 95.77 & 17090 & 92.92 & 17181 & 96.43 & 17061 & 92.92 & 17181 & 96.43 & 17061 \\
\hline $60-40 \%$ & 88.82 & 17293 & 79.92 & 18722 & 85.81 & 17517 & 79.92 & 18722 & 85.81 & 17517 & 79.92 & 18722 & 85.89 & 17523 & 79.92 & 18722 & 85.89 & 17524 \\
\hline $80-20 \%$ & 71.55 & 19472 & 71,13 & 19748 & 74.79 & 18721 & 71.13 & 19748 & 74.79 & 18721 & 71.13 & 19748 & 74.79 & 18721 & 71.13 & 19745 & 74.79 & 18721 \\
\hline
\end{tabular}


After solving the CP models including the WAFGP method of these lower and upper level problems, feasible fuzzy-stochastic solutions which trade-off between makespan and total resource cost objectives are obtained as given in Table 15 under 2-hours runtime limit. In addition to fuzzy objective function values of these compromise solutions, the individual membership function values and the weighted additive membership values (total satisfaction degrees) for each sub-problem, i.e., middle, lower and upper are also involved in Table 15. Furthermore, optimization details such as the number of variables and constraints, memory usages, solver iterations etc. for both ILP and CP-based transformation approaches are included at the end of this table.

When results of the ILP and CP-based approaches are compared, it is recognized that ILPbased approach cannot generate any feasible solution for most of the objective weights under 2-hours runtime limit. As mentioned before, any feasible solution cannot also be produced by this ILP-based approach even for 12-hours runtime limit. Actually, this ILP-based approach can just find a feasible solution for the objective weights of $w_{1}=0.8 \& w_{2}=0.2$. However, it is clearly seen in Table 15 that CP-based approach has found a superior result, i.e., (50.19, $71.14,92.02$ ) for the fuzzy expected project makespan objective when compared to the result of ILP-based approach $(52.55,72.73,94.71)$. When the generated compromise solutions for these objective weights $\left(w_{1}=0.8 \& w_{2}=0.2\right)$ are compared to each other, it can be concluded that $\mathrm{CP}$-based approach is able to provide better results for the project makespan objective within 2-hours runtime limit. In a nutshell, when the project managers declared that the total resource cost objective is relatively much more important than the project makespan objective, any compromise solution cannot be found by the ILP-based approach in the multiobjective optimization phase of the proposed methodology. In order to overcome this problematic issue, CP-based transformation approaches can be very useful to find several compromise solutions easily within reasonable computation times. Moreover, it is clearly seen in Table 15 that the model dimension including the numbers of decision variables and constraints can be significantly reduced by making use of such a CP-based transformation approach. This computational case study has shown that CP-based transformation approaches can be very useful for solving realistic size fuzzy-stochastic combinatorial optimization problems in reasonable computation times.

Lastly, when we evaluated the results of CP-based approach on their own merits, it can be concluded that except the solution for the objective weights of $w_{1}=0.4 \& w_{2}=0.6$, all of the generated results with other weights can be stated as compromise solutions since they are not dominated by each other. In other words, when the project makespan gets longer, total resource cost value will decrease. In the opposite case, total resource cost objective will deteriorate in case of shorter project makespan values. However, most likely and pessimistic values $(96.86$ versus 16841 and 119.5 versus 17947) of the two objectives for the objective weights of $w_{1}=$ $0.4 \& w_{2}=0.6$ are dominated by the other solution whose project makespan and total resource cost values are equal to 96.68 versus 16726 and 118.1 versus 17813, respectively. On the other hand, it should also be highlighted here that optimistic values of these solutions (71.72 versus $14694 \& 79.31$ versus 14382) are not dominated by each other (see in Table 15). The dominance relations among these solutions can be more accurately evaluated after obtaining the optimal results. It should also be noted here that the presented solutions in Table 15 are not optimal since both of the ILP and CP-based approaches were terminated at 2-hours runtime limit.

Finally, ERP company's managers and consultants have announced that all of these solutions presented in Table 15 are applicable and therefore, they can be realized while generating appropriate project schedules under such a fully uncertain environments. Moreover, they also reached agreement with the customer firm's managers to apply one of these solutions. Indeed, they achieved a consensus on the compromise solution with the objective weights of $w_{1}=$ $0.6 \& w_{2}=0.4$ 
Table 15 Comparison of the multi-objective optimization results of the proposed ILP and CP-based approaches with WAFGP

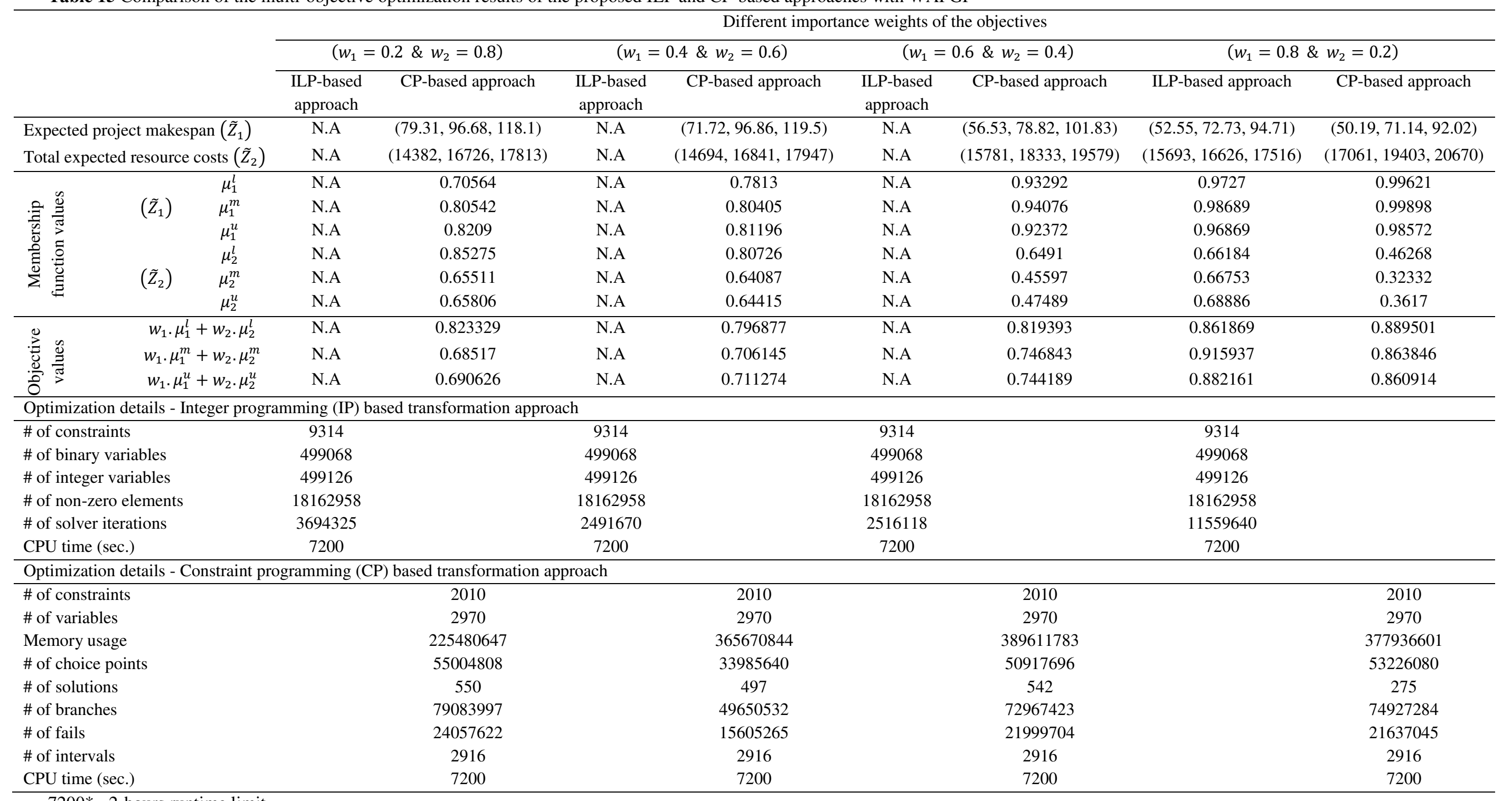


Indeed, they thought that project makespan objective is a little more important than the resource costs. For that reason, they have chosen that solution with the objective weights of $w_{1}=0.6 \& w_{2}=0.4$ because it is more practical to implement. Thus, some of the managerial results of this solution is visualized in the remainder part of this section. Firstly, the fuzzy project makespan values under different scenarios are presented in Table 16. According to this table, scenarios\#11 \& 1 have the minimum and maximum project makespan values, respectively. For this reason, Gantt charts and some of the resource profiles related to only these scenarios were drawn in Figs. 17 - 20. Details of the fuzzy completion times of the project activities with mode selections are also given in Appendices C and D. With the help of these completion times and fuzzy durations of the activities, their starting time intervals are determined and displayed in Figs. 17 and 18. It should be noted here that starting and completion time intervals may intersect with each other for most of the project activities. In other words, a project activity may be initialized or completed at this intersection time points. Actually, these intersections are resulted from fully uncertain nature of the examined problem.

Table 16 Fuzzy project makespan values under different scenarios $\left(\mathrm{w}_{1}=0.6 \& \mathrm{w}_{2}=0.4\right)$

\begin{tabular}{cccc}
\hline Scenario & Fuzzy project makespan & Scenario & Fuzzy project makespan \\
\hline 1 & $(61,85,108)$ & 10 & $(60,83,108)$ \\
2 & $(57,79,104)$ & 11 & $(50,71,92)$ \\
3 & $(59,83,105)$ & 12 & $(57,79,103)$ \\
4 & $(53,75,98)$ & 13 & $(56,79,102)$ \\
5 & $(58,81,104)$ & 14 & $(56,78,102)$ \\
6 & $(59,81,105)$ & 15 & $(53,75,98)$ \\
7 & $(57,78,99)$ & 16 & $(55,78,100)$ \\
8 & $(55,76,98)$ & 17 & $(59,82,105)$ \\
9 & $(61,83,108)$ & 18 & $(60,84,107)$ \\
\hline
\end{tabular}

After drawing the fuzzy compromise project schedules under scenarios\#1 \& 11, some resource profiles of the renewable project sources, i.e., human resources are displayed in Figs. 19 - 20. In detail, resource profile of the process experts in customer firm are shown in Fig. 19 by considering just optimistic case under scenario\#11. Actually, this resource profile is obtained from the solution of lower level problem only. In a similar way, resource profiles of this type of human resource can also be visualized for most likely and pessimistic cases by using the solutions of middle and upper level problems. In Fig. 20, resource profile of the ERP Company's consultants are depicted by considering just most likely case under scenario\#1. Similarly, optimistic and pessimistic resource profiles of this kind of human resource can also be drawn by using the solutions of lower and upper level problems.

Ultimately, availability levels of the renewable and non-renewable project resources are demonstrated in Figs. 21 - 23. In Fig. 21, fuzzy availability levels of the ERP consultants are shown under different scenarios. Similarly, fuzzy availability levels of the process experts are also displayed in Fig. 22. According to Fig. 21-22, middle and upper level problems gave the same results for the human resources. In other words, availability levels of the human resources have the same values in most likely and pessimistic cases. In Fig. 23, availability levels of the non-renewable resource, i.e., project budget needed under different scenarios are portrayed.

It should be noted here that crisp project funds/budget are provided under each scenario despite of defining them as fuzzy variables. In contrast to the human resource costs, availability levels of this project budget are not multiplied by a cost term while calculating total resource costs. Actually, this is the main reason why crisp project budget values are obtained under each scenario instead of fuzzy ones. 
Time Periods (Days)

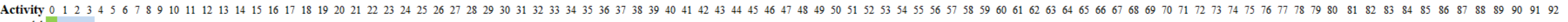

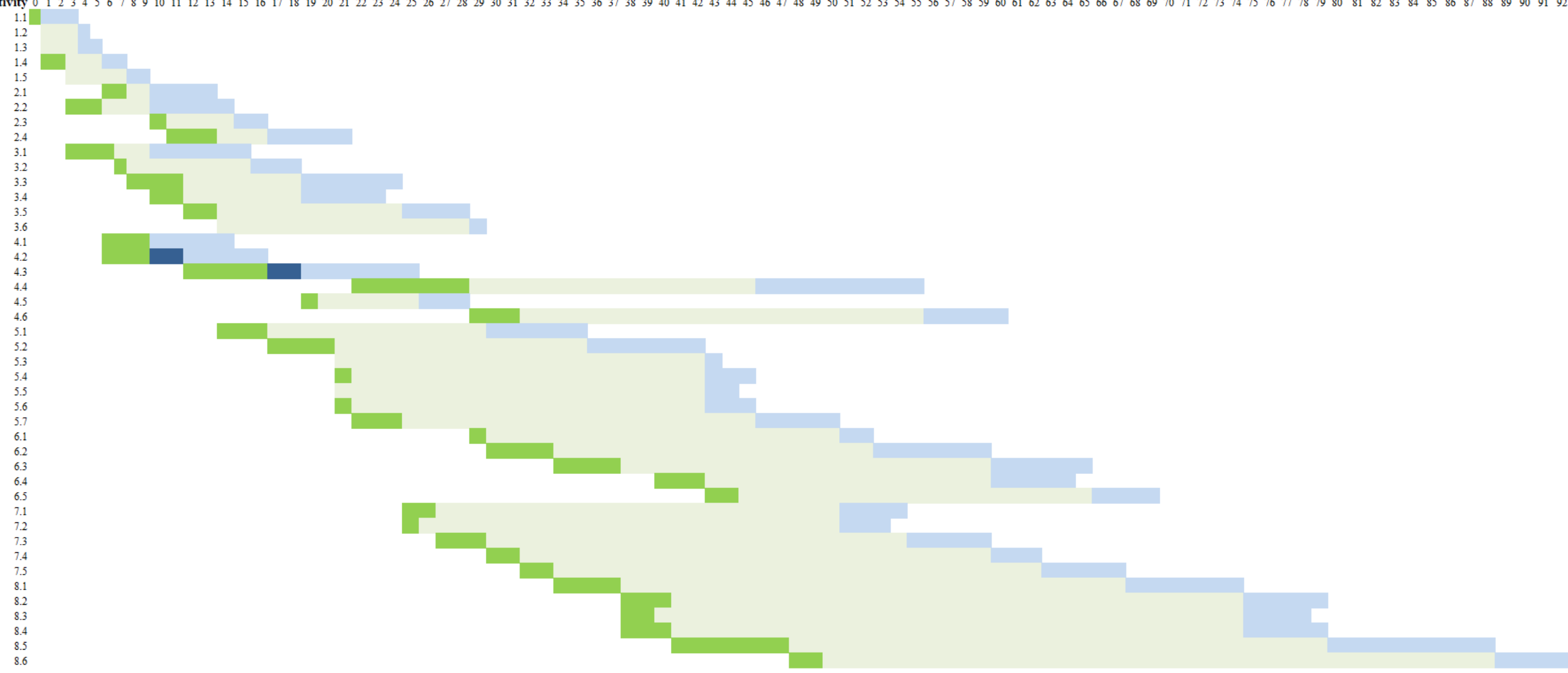

Completion time interval of a project activity

Starting time interval of a project activity

Certain activity duration

Intersection of starting and completion time intervals

Fig. 17 Gantt chart of a fuzzy compromise project schedule with minimum makespan under scenario\#11 $\left(\mathrm{w}_{1}=0.6 \& \mathrm{w}_{2}=0.4\right)$ 
Time Periods (Days)

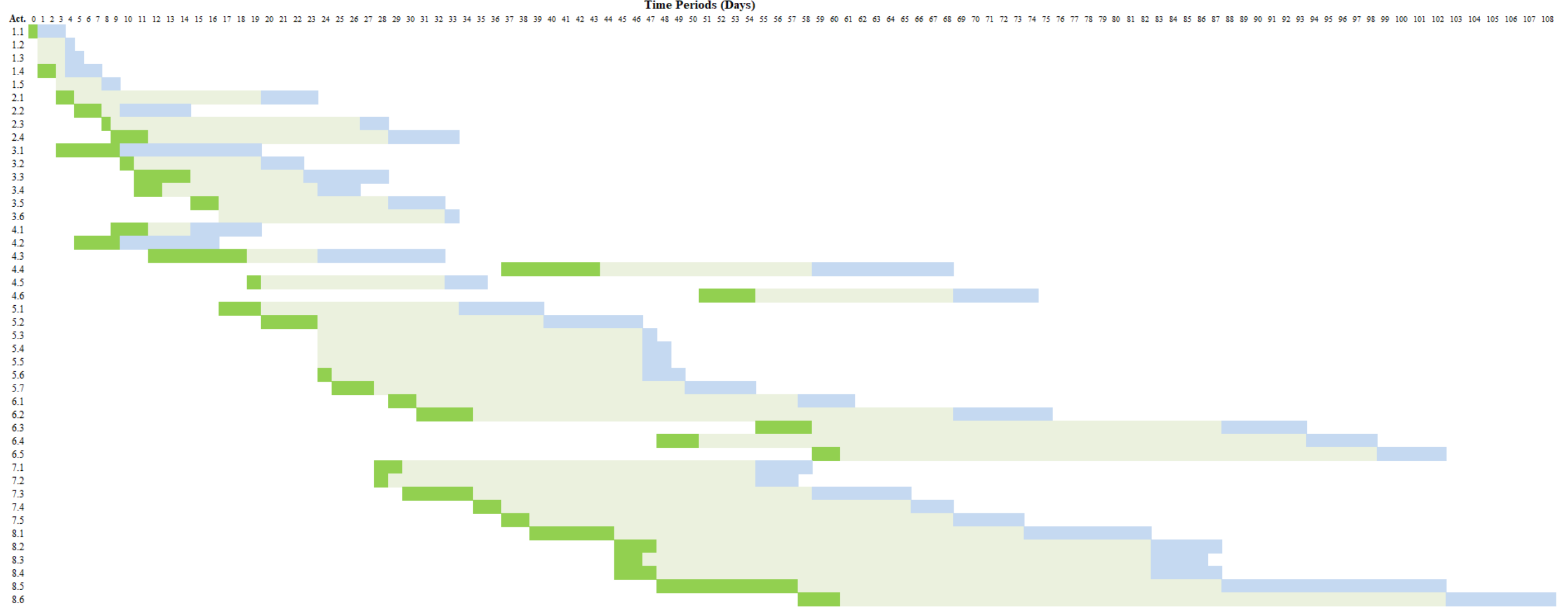

Fig. 18 Gantt chart of a fuzzy compromise project schedule with maximal makespan under scenario\#1 $\left(\mathrm{w}_{1}=0.6 \& \mathrm{w}_{2}=0.4\right)$ 


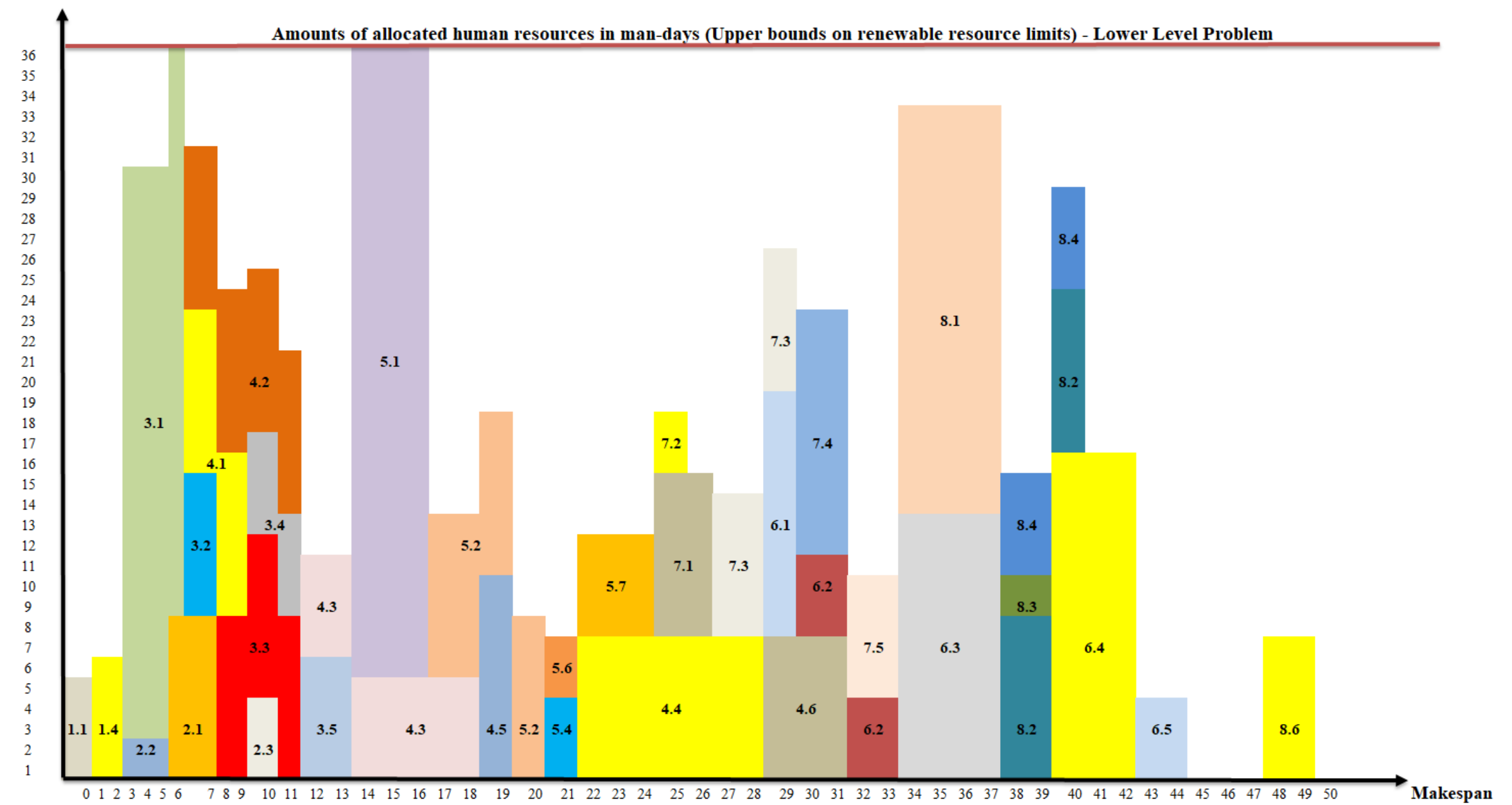

Fig. 19 The resource profile of the process experts (human resources) for the optimistic case under scenario\#11 $\left(\mathrm{w}_{1}=0.6 \& \mathrm{w}_{2}=0.4\right)$ 


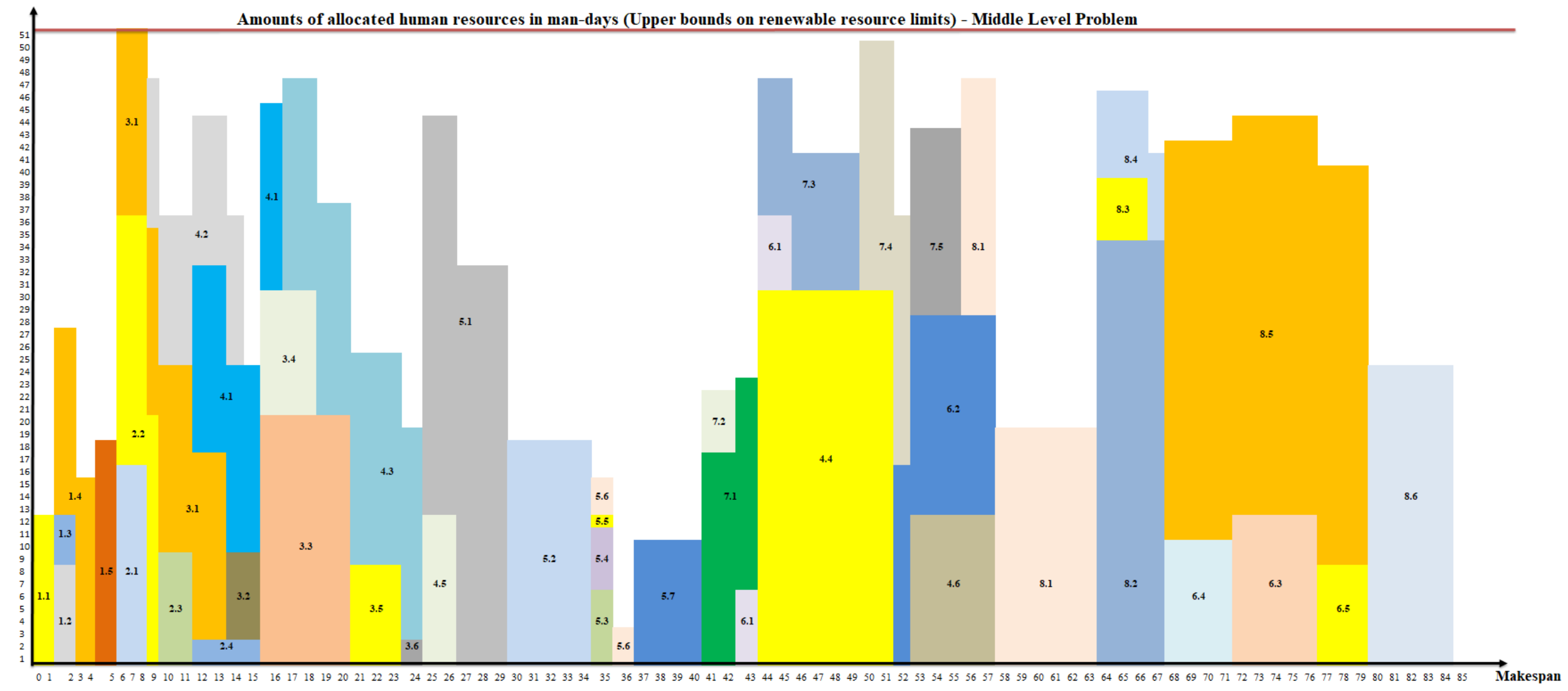

Fig. 20 The resource profile of the ERP Company's consultants (human resources) for the most likely case under scenario\#1 $\left(\mathrm{w}_{1}=0.6 \& \mathrm{w}_{2}=0.4\right)$ 
Fuzzy availability levels of ERP consultants

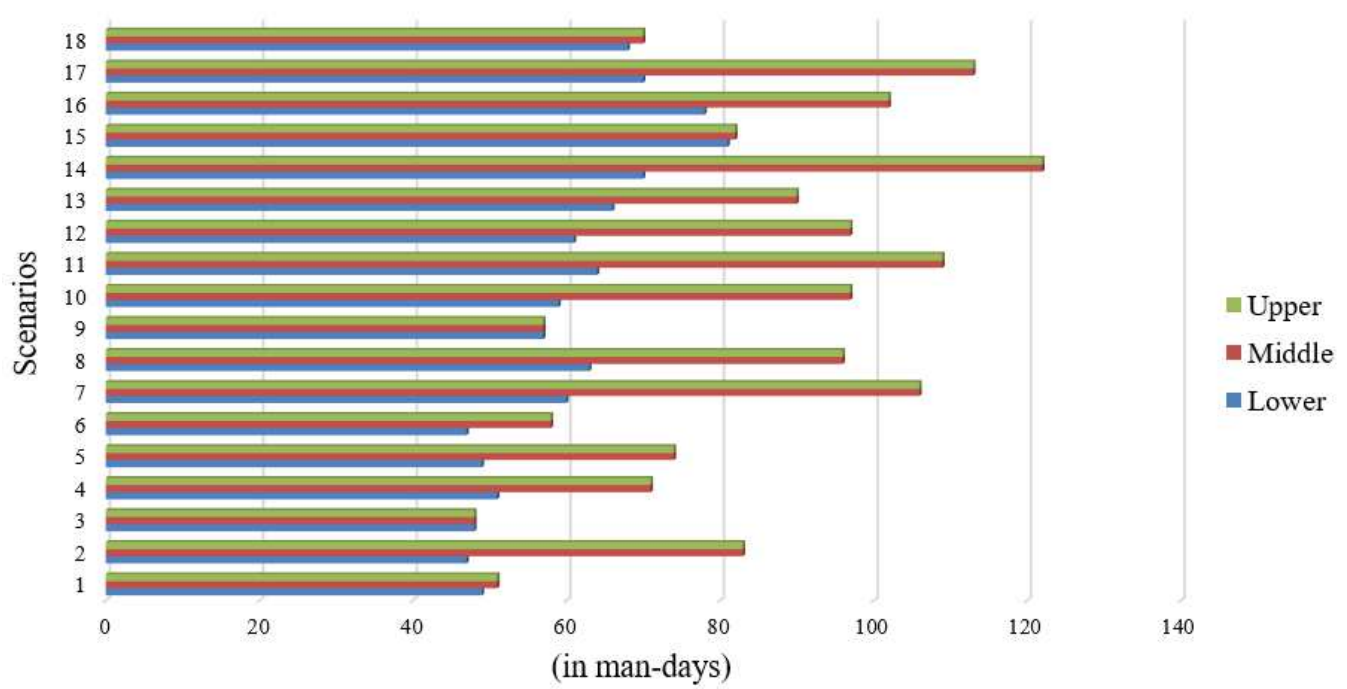

Fig. 21 Fuzzy availability levels of human resources (ERP company's consultants) under different scenarios

Fuzzy availability levels of process experts

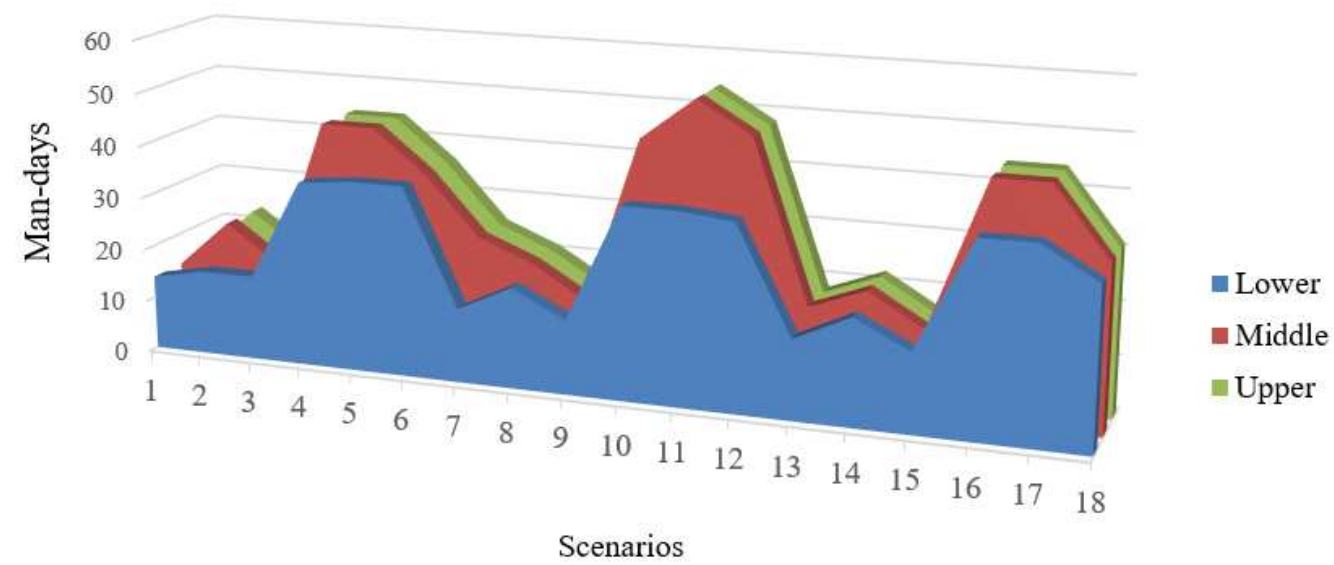

Fig. 22 Fuzzy availability levels of human resources (process experts) under different scenarios

Availability levels of non-renewable project resource

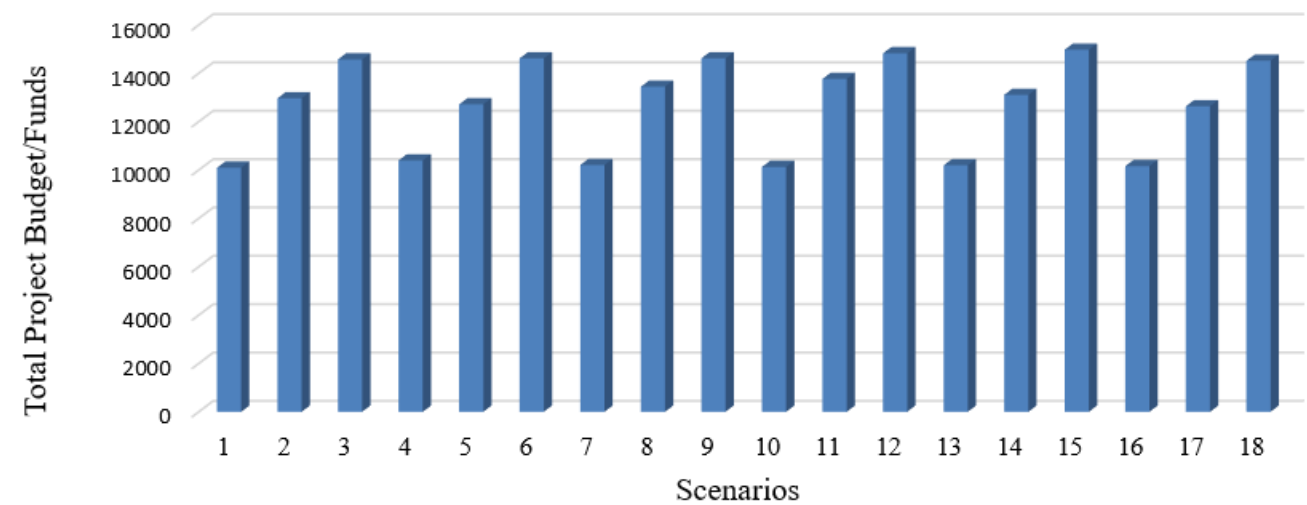

Fig. 23 Availability levels of project funds/budget as a non-renewable resource under different scenarios 


\section{Concluding remarks \& future researches}

This paper introduces a novel CP-based transformation approach with a bound \& decomposition principle for solving a multi-objective and multi-mode RIPSP under mixed fuzzy-stochastic environments. Actually, all of the project input data are represented by either fuzzy or stochastic parameters for better reflection of the real-life settings. Because of the fully uncertain nature of the project data, it is also aimed to generate fuzzy-stochastic outputs which correspond to project schedules and availability levels of the renewable/non-renewable project resources. Due the NP-complete nature of the examined problem, it is a challenging task to produce optimal project schedules within reasonable computation times even under fully deterministic environments. Therefore, development of a CP-based transformation approach is intended by this research instead of a classical ILP-based one to solve the examined problem in reasonable computing times. It should also be noted here that most of the transformation approaches in fuzzy mathematical programming literature usually utilize an ILP-based approach with fuzzy arithmetic operations and fuzzy ranking techniques. In recent years, it was also proven that these approaches may cause questionable results due to some problematic issues resulting from the usage of standard fuzzy arithmetic. Furthermore, these traditionally ILP-based transformation approaches may also be inadequate while solving large-size combinatorial optimization problems under such a mixed fuzzy-stochastic uncertainties. Based on this motivation, the main aim of this research is to develop a CP-based transformation approach with a bound \& decomposition principle which does not require fuzzy arithmetic and ranking operators directly. The proposed approach is able to produce fuzzy project schedules and fuzzy resource availabilities under different scenarios. In order to cope with the multiobjective nature of the problem, the proposed transformation approach is also integrated with WAFGP method. The validity and practicality of the proposed approach is tested on a realworld application to a production-and-operations management (POM) module implementation process of an international Enterprise Resource Planning (ERP) software. The generated results by the proposed CP-based transformation approach are also compared to a similar ILP-based apporach. Computational results have shown that the proposed CP-based approach outperforms than the ILP-based one in terms of both solution quality and computing times. Development of a matheuristic solution approach which hybridizes a meta-heuristic algorithm with the proposed $\mathrm{CP}$ models can be scheduled as a future work in order to solve the larger scaled problem instances within shorter runtimes. Moreover, fuzzy-random variables can also be employed to describe some project data for better reflection of the hybrid uncertainties in the future studies.

\section{Declarations}

Conflict of interest The author declares that they have no conflict of interest.

Ethical approval This article does not contain any studies with human participants or animals performed by any of the authors. 


\section{Appendices}

Appendix A. Fuzzy project schedules under different scenarios for the illustrative example

\begin{tabular}{|c|c|c|c|c|c|c|}
\hline \multirow[t]{2}{*}{ Scenario } & \multicolumn{3}{|c|}{ IP-based approach } & \multicolumn{3}{|c|}{ CP-based approach } \\
\hline & Activity & Mode & Fuzzy completion time & Activity & Mode & Fuzzy completion time \\
\hline \multirow{5}{*}{1} & 1 & 1 & $(3,4,5)$ & 1 & 1 & $(3,4,5)$ \\
\hline & 2 & 1 & $(2,2,4)$ & 2 & 1 & $(1,2,3)$ \\
\hline & 3 & 2 & $(4,5,6)$ & 3 & 2 & $(4,5,6)$ \\
\hline & 4 & 1 & $(5,6,8)$ & 4 & 1 & $(4,6,8)$ \\
\hline & 5 & 1 & $(5,7,9)$ & 5 & 1 & $(5,7,9)$ \\
\hline \multirow{5}{*}{2} & 1 & 2 & $(5,6,7)$ & 1 & 2 & $(5,6,7)$ \\
\hline & 2 & 1 & $(1,2,4)$ & 2 & 1 & $(1,2,3)$ \\
\hline & 3 & 2 & $(4,5,6)$ & 3 & 2 & $(4,5,6)$ \\
\hline & 4 & 1 & $(6,8,10)$ & 4 & 1 & $(6,8,10)$ \\
\hline & 5 & 1 & $(5,7,9)$ & 5 & 1 & $(5,7,9)$ \\
\hline \multirow{5}{*}{3} & 1 & 1 & $(3,4,5)$ & 1 & 1 & $(3,4,5)$ \\
\hline & 2 & 1 & $(2,2,4)$ & 2 & 1 & $(1,2,3)$ \\
\hline & 3 & 2 & $(4,5,6)$ & 3 & 2 & $(4,5,6)$ \\
\hline & 4 & 1 & $(5,6,8)$ & 4 & 1 & $(4,6,8)$ \\
\hline & 5 & 1 & $(5,7,9)$ & 5 & 1 & $(5,7,9)$ \\
\hline \multirow{5}{*}{4} & 1 & 1 & $(3,4,5)$ & 1 & 1 & $(3,4,5)$ \\
\hline & 2 & 1 & $(1,2,4)$ & 2 & 1 & $(1,2,3)$ \\
\hline & 3 & 2 & $(4,5,6)$ & 3 & 2 & $(4,5,6)$ \\
\hline & 4 & 1 & $(5,6,8)$ & 4 & 1 & $(4,6,8)$ \\
\hline & 5 & 1 & $(5,7,9)$ & 5 & 1 & $(5,7,9)$ \\
\hline \multirow{5}{*}{5} & 1 & 1 & $(3,4,5)$ & 1 & 1 & $(3,4,5)$ \\
\hline & 2 & 1 & $(4,9,12)$ & 2 & 1 & $(4,6,8)$ \\
\hline & 3 & 1 & $(6,7,9)$ & 3 & 1 & $(6,9,12)$ \\
\hline & 4 & 1 & $(4,9,12)$ & 4 & 1 & $(4,6,8)$ \\
\hline & 5 & 1 & $(7,11,15)$ & 5 & 1 & $(7,11,15)$ \\
\hline \multirow{5}{*}{6} & 1 & 1 & $(7,9,11)$ & 1 & 1 & $(7,9,11)$ \\
\hline & 2 & 1 & $(1,4,4)$ & 2 & 1 & $(1,2,3)$ \\
\hline & 3 & 2 & $(4,5,6)$ & 3 & 2 & $(4,5,6)$ \\
\hline & 4 & 1 & $(10,13,14)$ & 4 & 1 & $(8,11,14)$ \\
\hline & 5 & 2 & $(10,13,16)$ & 5 & 2 & $(10,13,16)$ \\
\hline \multirow{5}{*}{7} & 1 & 1 & $(3,4,5)$ & 1 & 1 & $(3,4,5)$ \\
\hline & 2 & 1 & $(4,9,12)$ & 2 & 1 & $(4,6,8)$ \\
\hline & 3 & 1 & $(6,7,9)$ & 3 & 2 & $(7,9,11)$ \\
\hline & 4 & 1 & $(4,9,12)$ & 4 & 1 & $(5,8,11)$ \\
\hline & 5 & 1 & $(7,11,15)$ & 5 & 1 & $(8,11,14)$ \\
\hline \multirow{5}{*}{8} & 1 & 1 & $(3,4,5)$ & 1 & 1 & $(3,4,5)$ \\
\hline & 2 & 1 & $(4,6,8)$ & 2 & 1 & $(5,8,11)$ \\
\hline & 3 & 2 & $(7,9,11)$ & 3 & 2 & $(7,9,11)$ \\
\hline & 4 & 1 & $(7,8,11)$ & 4 & 1 & $(4,6,8)$ \\
\hline & 5 & 1 & $(8,11,14)$ & 5 & 1 & $(8,11,14)$ \\
\hline \multirow{5}{*}{9} & 1 & 1 & $(3,4,5)$ & 1 & 1 & $(3,4,5)$ \\
\hline & 2 & 1 & $(2,3,4)$ & 2 & 1 & $(1,2,3)$ \\
\hline & 3 & 1 & $(2,3,4)$ & 3 & 1 & $(2,3,4)$ \\
\hline & 4 & 1 & $(4,6,8)$ & 4 & 1 & $(4,6,8)$ \\
\hline & 5 & 1 & $(4,5,7)$ & 5 & 1 & $(3,5,7)$ \\
\hline \multirow{5}{*}{10} & 1 & 2 & $(5,6,7)$ & 1 & 2 & $(5,6,7)$ \\
\hline & 2 & 1 & $(1,3,4)$ & 2 & 1 & $(1,2,3)$ \\
\hline & 3 & 1 & $(2,3,4)$ & 3 & 1 & $(2,3,4)$ \\
\hline & 4 & 1 & $(6,8,10)$ & 4 & 1 & $(6,8,10)$ \\
\hline & 5 & 2 & $(5,7,9)$ & 5 & 2 & $(5,7,9)$ \\
\hline \multirow{5}{*}{11} & 1 & 1 & $(3,4,5)$ & 1 & 1 & $(3,4,5)$ \\
\hline & 2 & 1 & $(2,3,4)$ & 2 & 1 & $(1,2,3)$ \\
\hline & 3 & 1 & $(2,3,4)$ & 3 & 1 & $(2,3,4)$ \\
\hline & 4 & 1 & $(4,6,8)$ & 4 & 1 & $(4,6,8)$ \\
\hline & 5 & 1 & $(3,5,7)$ & 5 & 1 & $(3,5,7)$ \\
\hline
\end{tabular}




\begin{tabular}{ccccccc}
\hline \multirow{6}{*}{12} & 1 & 1 & $(3,4,5)$ & 1 & 1 & $(3,4,5)$ \\
& 2 & 1 & $(3,3,4)$ & 2 & 1 & $(1,2,3)$ \\
& 3 & 2 & $(4,5,6)$ & 3 & 2 & $(4,5,6)$ \\
& 4 & 1 & $(5,6,8)$ & 4 & 1 & $(4,6,8)$ \\
& 5 & 1 & $\mathbf{( 5 , 7 , 9 )}$ & 5 & 1 & $\mathbf{( 5 , 7 , 9 )}$ \\
\hline \multirow{6}{*}{13} & 1 & 1 & $(4,6,8)$ & 1 & 1 & $(3,4,5)$ \\
& 2 & 1 & $(1,2,3)$ & 2 & 1 & $(4,6,8)$ \\
& 3 & 2 & $(4,5,6)$ & 3 & 2 & $(4,5,6)$ \\
& 4 & 1 & $\mathbf{( 5 , 8 , 1 1}$ & 4 & 1 & $(4,6,8)$ \\
& 5 & 1 & $(5,7,9)$ & 5 & 1 & $(\mathbf{5 , 8 , 1 1})$ \\
\hline \multirow{6}{*}{14} & 1 & 2 & $(5,6,7)$ & 1 & 2 & $(5,6,7)$ \\
& 2 & 1 & $(1,7,9)$ & 2 & 1 & $(5,7,9)$ \\
& 3 & 2 & $(5,5,6)$ & 3 & 2 & $(4,5,6)$ \\
& 4 & 1 & $(6,8,10)$ & 4 & 1 & $(6,8,10)$ \\
& 5 & 1 & $\mathbf{( 6 , 9 , 1 2}$ & 5 & 1 & $(\mathbf{6 , 9 , 1 2})$ \\
\hline \multirow{4}{*}{15} & 1 & 1 & $(3,4,5)$ & 1 & 1 & $(3,4,5)$ \\
& 2 & 1 & $(4,6,8)$ & 2 & 1 & $(4,6,8)$ \\
& 3 & 2 & $(4,5,6)$ & 3 & 2 & $(4,5,6)$ \\
& 4 & 1 & $(5,6,9)$ & 4 & 1 & $(4,6,8)$ \\
& 5 & 1 & $\mathbf{5 , 8 , 1 1}$ & 5 & 1 & $(\mathbf{5 , 8 , 1 1})$ \\
\hline \multirow{6}{*}{16} & 1 & 1 & $(3,4,5)$ & 1 & 1 & $(3,4,5)$ \\
& 2 & 1 & $(4,6,8)$ & 2 & 1 & $(4,6,8)$ \\
& 3 & 2 & $(4,5,6)$ & 3 & 2 & $(4,5,6)$ \\
& 4 & 1 & $(5,6,9)$ & 4 & 1 & $(4,6,8)$ \\
& 5 & 1 & $\mathbf{5 , 8 , 1 1}$ & 5 & 1 & $(\mathbf{5 , 8 , 1 1})$ \\
\hline
\end{tabular}

Appendix B. IBM ILOG CPLEX code of the used search strategies in the CP-based transformation approach execute \{

// Search strategy-1;

var $f=c p$. factory;

var phase1 = f.searchPhase(task, f.selectLargest $(f$. Impact ()$)$, f.selectLargest $(f$.valueSuccessRate ()$))$; var $f=c p$. factory;

var phase $2=f$.searchPhase $($ mode, $f . \operatorname{selectLargest}(f$. Impact ()$), f . \operatorname{selectLargest}(f$. valueSuccessRate ()$))$; cp.setSearchPhases (phase2, phase1);

// Search strategy-2;

var $f=c p$. factory;

var phase1 = f. searchPhase (task, f.selectLargest $(f$. Impact ()$)$, f.selectLargest (f.valueSuccessRate ()$))$;

var $f=c p$. factory;

var phase $2=f$. searchPhase $($ mode, $f . \operatorname{selectLargest}(f . \operatorname{Impact}()), f . \operatorname{selectLargest}(f$.valueSuccessRate ()$))$;

cp.setSearchPhases (phase1, phase 2$) ;\}$

// Search strategy-3;

var $f=c p$. factory;

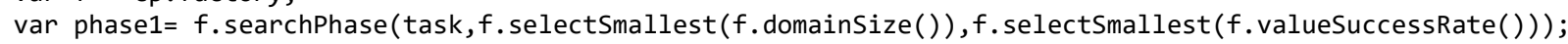
var $f=c p$. factory;

var phase $2=f$. searchPhase (mode, $f$. selectSmallest ( $f$.domainSize ( )), $f$. selectSmallest ( $f$.valueSuccessRate ()$)$ ); cp. setSearchPhases (phase1, phase 2$) ;\}$

// Search strategy-4;

var $f=c p$. factory;

var phase1= f.searchPhase(task,f.selectSmallest(f.domainSize( $))$, f. selectSmallest(f.valueSuccessRate( )));

var $f=c p$. factory;

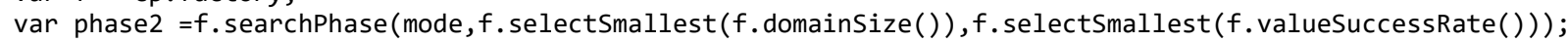

cp. setSearchPhases (phase2, phase1); \}

// Search strategy-5;

var $f=c p$. factory;

var phase $1=f$. searchPhase (task,f.selectLargest $(f . \operatorname{Impact}()), f . \operatorname{selectSmallest}(f . \operatorname{valueImpact}()))$;

var $f=c p$. factory;

var phase $2=f$. searchPhase $($ mode, $f . \operatorname{selectLargest}(f . \operatorname{Impact}()), f . \operatorname{selectSmallest}(f \cdot \operatorname{valueImpact}()))$;

cp. setSearchPhases (phase1, phase 2$) ;\}$

// Search strategy-6;

var $f=c p$. factory;

var phase1 $=f$. searchPhase $($ task, $f . \operatorname{selectLargest}(f . \operatorname{Impact}()), f . \operatorname{selectSmallest}(f . \operatorname{valueImpact}()))$;

var $f=c p$. factory;

var phase $2=f \cdot \operatorname{searchPhase}($ mode, $f . \operatorname{selectLargest}(f \cdot \operatorname{Impact}()), f . \operatorname{selectSmallest}(f \cdot \operatorname{valueImpact}()))$;

cp. setSearchPhases (phase2, phase1); \}

// Search strategy-7;

var $f=c p$. factory;

var phase1 = f. searchPhase $($ task,f.selectSmallest $(f . \operatorname{domainSize}())$, f. selectSmallest (f.valueImpact ()$))$; 


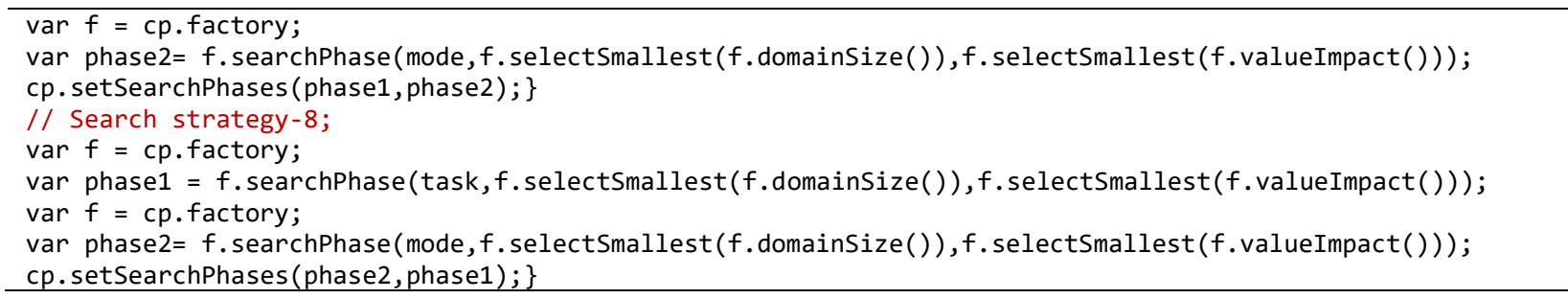

Appendix C. Compromise fuzzy project schedules under scenario\#11 via the proposed CP-based transformation approach for the real-life case study

\begin{tabular}{cccccc}
\hline Activity & Mode & Fuzzy completion times & Activity & Mode & Fuzzy completion times \\
\hline 1.1 & 1 & $(1,2,3)$ & 5.2 & 1 & $(21,32,42)$ \\
1.2 & 1 & $(1,3,4)$ & 5.3 & 1 & $(21,33,43)$ \\
1.3 & 1 & $(1,3,5)$ & 5.4 & 2 & $(22,34,45)$ \\
1.4 & 1 & $(3,5,7)$ & 5.5 & 2 & $(21,33,44)$ \\
1.5 & 1 & $(3,6,9)$ & 5.6 & 2 & $(22,34,45)$ \\
2.1 & 1 & $(8,9,13)$ & 5.7 & 1 & $(25,38,50)$ \\
2.2 & 1 & $(6,10,14)$ & 6.1 & 1 & $(30,40,52)$ \\
2.3 & 1 & $(11,12,16)$ & 6.2 & 3 & $(34,46,59)$ \\
2.4 & 3 & $(14,16,21)$ & 6.3 & 3 & $(38,51,65)$ \\
3.1 & 1 & $(7,11,15)$ & 6.4 & 3 & $(43,50,64)$ \\
3.2 & 2 & $(8,13,18)$ & 6.5 & 2 & $(45,54,69)$ \\
3.3 & 1 & $(12,18,24)$ & 7.1 & 2 & $(27,41,54)$ \\
3.4 & 3 & $(12,17,23)$ & 7.2 & 2 & $(26,40,53)$ \\
3.5 & 2 & $(14,21,28)$ & 7.3 & 1 & $(30,45,59)$ \\
3.6 & 1 & $(14,22,29)$ & 7.4 & 1 & $(32,48,62)$ \\
4.1 & 3 & $(10,11,14)$ & 7.5 & 1 & $(34,51,67)$ \\
4.2 & 3 & $(12,12,16)$ & 8.1 & 1 & $(38,56,74)$ \\
4.3 & 3 & $(19,20,25)$ & 8.2 & 1 & $(41,60,79)$ \\
4.4 & 2 & $(29,42,55)$ & 8.3 & 3 & $(40,59,78)$ \\
4.5 & 1 & $(20,22,28)$ & 8.4 & 3 & $(41,60,79)$ \\
4.6 & 1 & $(32,46,60)$ & 8.5 & 1 & $(48,68,88)$ \\
5.1 & 1 & $(17,27,35)$ & 8.6 & 1 & $(\mathbf{5 0 , 7 1 , 9 2 )}$ \\
\hline
\end{tabular}

Appendix D. Compromise fuzzy project schedules under scenario\#1 via the proposed CP-based transformation approach for the real-life case study

\begin{tabular}{cccccc}
\hline Activity & Mode & Fuzzy completion times & Activity & Mode & Fuzzy completion times \\
\hline 1.1 & 1 & $(1,2,3)$ & 5.2 & 1 & $(24,35,46)$ \\
1.2 & 1 & $(1,3,4)$ & 5.3 & 1 & $(24,36,47)$ \\
1.3 & 1 & $(1,3,5)$ & 5.4 & 1 & $(24,36,48)$ \\
1.4 & 1 & $(3,5,7)$ & 5.5 & 2 & $(24,36,48)$ \\
1.5 & 1 & $(3,6,9)$ & 5.6 & 2 & $(25,37,49)$ \\
2.1 & 1 & $(5,9,23)$ & 5.7 & 1 & $(28,41,54)$ \\
2.2 & 1 & $(8,10,14)$ & 6.1 & 2 & $(31,46,61)$ \\
2.3 & 1 & $(9,12,28)$ & 6.2 & 3 & $(35,58,75)$ \\
2.4 & 3 & $(12,16,33)$ & 6.3 & 3 & $(59,77,93)$ \\
3.1 & 3 & $(10,14,19)$ & 6.4 & 3 & $(51,72,98)$ \\
3.2 & 2 & $(11,16,22)$ & 6.5 & 2 & $(61,80,102)$ \\
3.3 & 1 & $(15,21,28)$ & 7.1 & 2 & $(30,44,58)$ \\
3.4 & 2 & $(13,19,26)$ & 7.2 & 2 & $(29,43,57)$ \\
3.5 & 2 & $(17,24,32)$ & 7.3 & 3 & $(35,50,65)$ \\
3.6 & 1 & $(17,25,33)$ & 7.4 & 1 & $(37,53,68)$ \\
4.1 & 3 & $(12,17,19)$ & 7.5 & 1 & $(39,56,73)$ \\
4.2 & 3 & $(10,15,16)$ & 8.1 & 3 & $(45,64,82)$ \\
4.3 & 3 & $(19,25,32)$ & 8.2 & 1 & $(48,68,87)$ \\
4.4 & 2 & $(44,52,68)$ & 8.3 & 3 & $(47,67,86)$ \\
4.5 & 1 & $(20,27,35)$ & 8.4 & 3 & $(48,68,87)$ \\
4.6 & 2 & $(55,58,74)$ & 8.5 & 3 & $(58,80,102)$ \\
5.1 & 1 & $(20,30,39)$ & 8.6 & 2 & $(\mathbf{6 1 , 8 5 , 1 0 8 )}$ \\
\hline
\end{tabular}




\section{References}

Alipouri Y, Sebt MH, Ardeshir A, Chan WT (2019) Solving the FS-RCPSP with hyperheuristics: A policy-driven approach. Journal of the Operational Research Society 70(3): 403-419.

Alipouri Y, Sebt MH, Ardeshir A, Zarandi MHF (2020) A mixed-integer linear programming model for solving fuzzy stochastic resource constrained project scheduling problem. Operational Research 20: 197-217.

Amid A, Ghodsypour SH, O'Brien C (2009) A weighted additive fuzzy multiobjective model for the supplier selection problem under price breaks in a supply chain. International Journal of Production Economics 121: 323-332.

Artykov D, Atymtayeva L (2015) A fuzzy linear programming approach for resourceconstrained project scheduling. Advanced Engineering Technology and Application 4(3): 47-52.

Artigues C, Leus R, Nobibon FT (2013) Robust optimization for resource-constrained project scheduling with uncertain activity durations. Flexible Services and Manufacturing Journal 25:175-205.

Ashtiani B, Leus R, Aryanezhad MB (2011) New competitive results for the stochastic resource-constrained project scheduling problem: Exploring the benefits of preprocessing. Journal of Scheduling 14 (2): 157-171.

Atli O, Kahraman C (2012) Fuzzy resource-constrained project scheduling using taboo search algorithm. International Journal of Intelligent Systems 27(10): 873-907.

Atli O, Kahraman C (2014) Resource-constrained project scheduling problem with multiple execution modes and fuzzy/crisp activity durations. Journal of Intelligent \& Fuzzy Systems 26(4): 2001-2020.

Balouka N, Cohen I (2021) A robust optimization approach for the multi-mode resourceconstrained project scheduling problem. European Journal of Operational Research 291(2): 457-470.

Baykasoğlu A, Subulan K (2015) An analysis of fully fuzzy linear programming with fuzzy decision variables through logistics network design problem. Knowledge-Based Systems 90: 165-184.

Baykasoğlu A, Subulan K (2016) A multi-objective sustainable load planning model for intermodal transportation networks with a real-life application. Transportation Research Part E 95:207-247.

Baykasoğlu A, Subulan K (2017) Constrained fuzzy arithmetic approach to fuzzy transportation problems with fuzzy decision variables. Expert Systems with Applications 81:193-222.

Baykasoğlu A, Subulan K (2019) A direct solution approach based on con- strained fuzzy arithmetic and metaheuristic for fuzzy transportation problems. Soft Computing 23(5): 1667-1698.

Baykasoğlu A, Dudaklı N, Şenol ME, Kömürcü F (2020) Mathematical programming approach to productivity improvement in wind turbine-blade manufacturing through a case study. Engineering with Computers https://doi.org/10.1007/s00366-020-01044-5

Bellman R, Zadeh LA (1970) Decision-making in a fuzzy environment. Management Science 17:141-164.

Berthold T, Heinz S, Lübbecke ME, Möhring RH, Schulz J (2010) A constraint integer programming approach for resource-constrained project scheduling. In: 7th International Conference on Integration of AI and OR Techniques in Constraint Programming, CPAIOR, Bologna, Italy, LNCS, vol. 6140, pp. 51-55.

Bhaskar T, Pal M N, Pal AK (2011) A heuristic method for RCPSP with fuzzy activity times. European Journal of Operational Research 208:57-66. 
Birjandi A, Mousavi SM (2019) Fuzzy resource-constrained project scheduling with multiple routes: A heuristic solution. Automation in Construction 100: 84-102.

Bruni ME, Pugliese LDP, Beraldi P, Guerriero F (2018) A computational study of exact approaches for the adjustable robust resource-constrained project scheduling problem. Computers \& Operations Research 99:178-190.

Chand S, Singh HK, Ray T (2016) Finding robust solutions for resource constrained project scheduling problems involving uncertainties. In: 2016 IEEE Congress on Evolutionary Computation (CEC), Vancouver, Canada, 24-29 July.

Chakrabortty RK, Sarker RA, Essam D L (2017) Resource constrained project scheduling with uncertain activity durations. Computers \& Industrial Engineering 112:537-550.

Chen L, Zhang Z (2016) Preemption resource-constrained project scheduling problems with fuzzy random duration and resource availabilities. Journal of Industrial and Production Engineering 33(6): 373-382.

Chen Z, Demeulemeester E, Bai S, Guo Y (2018) Efficient priority rules for the stochastic resource-constrained project scheduling problem. European Journal of Operational Research 270(3): 957-967.

Chen H, Ding G, Zhang J, Qin S (2019) Research on priority rules for the stochastic resource constrained multi-project scheduling problem with new project arrival. Computers \& Industrial Engineering 137:106060.

Chen H, Ding G, Qin S, Zhang J, (2021) A hyper-heuristic based ensemble genetic programming approach for stochastic resource constrained project scheduling problem. Expert Systems with Applications 167: 114174.

Drexl A, Kimms A (2001) Optimization guided lower and upper bounds for the resource investment problem. Journal of the Operational Research Society 52:340-351.

Gang J, Xu J, Xu Y (2013) Multiproject resources allocation model under fuzzy random environment and its application to industrial equipment installation engineering. Journal of Applied Mathematics Article ID 818731, 1-19.

Hapke M, Jaszkiewicz A, Slowinski R (1994) Fuzzy project scheduling system for software development. Fuzzy Sets Systems 21: 101-117.

Hapke M, Slowinski R (1996) Fuzzy priority heuristics for project scheduling. Fuzzy Sets Systems 83:291-299.

Hauder VA, Beham A, Raggl S, Parragh SN, Affenzeller M (2020) Resource-constrained multiproject scheduling with activity and time flexibility. Computers \& Industrial Engineering 150:106857.

Hsu CC, Kim DS (2005) A new heuristic for the multi-mode resource investment problem. Journal of the Operational Research Society 56(4):406-413.

Huang W, Ding L, Wen B, Cao B (2009) Project scheduling problem for software development with random fuzzy activity duration times. Springer, Berlin.

Huang W, Oh SK, Pedrycz W (2013) A fuzzy time-dependent project scheduling problem. Information Sciences 246:100-114.

IAS Inc. Industrial Application Software, CANIAS ERP. https://www.canias40.com/tr [accessed 9 June 2021].

IBM (2017) IBM ILOG CPLEX optimization studio Version 12 Release 8 user's manual.

Javanmard S, Afshar-Nadjaf B, Niaki STA (2017) Preemptive multi-skilled resource investment project scheduling problem: Mathematical modelling and solution approaches. Computers and Chemical Engineering 96:55-68.

Jayalakshmi M, Pandian P (2012) A new method for finding an optimal fuzzy solution for fully fuzzy linear programming problems. International Journal of Engineering Research and Applications 2(4):247-254. 
Jedrzejowicz P, Ratajczak-Ropel E (2019) Experimental evaluation of a-teams solving resource availability cost problem. Intelligent Decision Technologies 142: 213-223.

Jørgensen T (1999) Project scheduling as a stochastic dynamic decision problem $\mathrm{PhD}$ Thesis. Trondheim, Norway: Norwegian University of Science and Technology.

Kaveh A, Khanzadi M, Alipour M (2016) Fuzzy resource constraint project scheduling problem using CBO and CSS algorithms. International Journal of Civil Engineering 14(5): 325-337.

Ke H, Liu B (2007) Project scheduling problem with mixed uncertainty of randomness and fuzziness. European Journal of Operational Research 183(1):135-147.

Ke H, Ma W, Ma J (2012) Solving project scheduling problem with the philosophy of fuzzy random programming. Fuzzy Optimization and Decision Making 11:269-284.

$\mathrm{Ke} \mathrm{H}$, Ma J (2014) Modeling project time-cost trade-off in fuzzy random environment. Applied Soft Computing 19:80-85.

Khalilzadeh M, Shakeri H, Gholami H, Amini L (2017) A heuristic algorithm for project scheduling with fuzzy parameters. Procedia Computer Science 121:63-71.

Kolisch R, Drexl A (1997) Local search for non-preemptive multi-mode resource constrained project scheduling. IIE Transactions 29: 987-999.

Kong F, Dou D (2021) Resource-Constrained Project Scheduling Problem under Multiple Time Constraints. Journal of Construction Engineering and Management 147(2):04020170.

Knyazeva M, Bozhenyuk A, Rozenberg I (2015) Resource-constrained project scheduling approach under fuzzy conditions. Procedia Computer Science, 77:56-64.

Li H, Womer N (2015) Solving stochastic resource-constrained project scheduling problems by closed-loop approximate dynamic programming. European Journal of Operational Research 246:20-33.

Liess O, Michelon P (2008) A constraint programming approach for the resource-constrained project scheduling problem. Annals of Operations Research 157:25-36.

Liu S, Yung KL, Ip WH (2007) Genetic local search for resource-constrained project scheduling under uncertainty. Information and Management Sciences 18(4):347-363.

Liu SX, Song JH (2011) Combination of constraint programming and mathematical programming for solving resources-constrained project-scheduling problems. Control Theory \& Applications 28(8):1113-1120.

Long LD, Ohsato A (2008) Fuzzy critical chain method for project scheduling under resource constraints and uncertainty. International Journal of Project Management 26(6):688-698.

Mahdavi A, Shirazi B, Rezaeian J (2021) Toward a scalable type-2 fuzzy model for resourceconstrained project scheduling problem. Applied Soft Computing 100:106988.

Malcolm DG, Rosenbloom JM, Clark, CE, Fazar W (1959) Application of a technique for research and development program evaluation. Operations Research 7:646-669.

Masmoudi M, Hait A (2013) Project scheduling under uncertainty using fuzzy modelling and solving techniques. Engineering Applications of Artificial Intelligence 26:135-149.

Merrikhi E, Najafi AB, Shahsavar A (2018) Project resource investment problem under progress payment model. Journal of Industrial and Systems Engineering 11(3):84-101.

Motwani J, Mirchandani D, Madan M, Gunesekaran A (2002) Successful implementation of ERP projects: Evidence from two case studies. International Journal of Production Economics 75(1-2):83-96.

Najafi AA, Azimi F (2009) A priority rule-based heuristic for resource investment project scheduling problem with discounted cash flows and tardiness penalties. Mathematical Problems in Engineering doi: 10.1155/2009/106425.

Najafi AA, Niaki STA (2006) A genetic algorithm for resource investment problem with discounted cash flows. Applied Mathematics and Computation 183(2):1057-1070.

Nematian J, Eshghi K, Eshragh-Jahromi A (2010) A resource-constrained project scheduling problem with fuzzy random duration. Journal of Uncertain Systems 4(2):123-132. 
Okubo H, Miyamoto T, Yoshida S, Mori K, Kitamura S, Izui Y (2015) Project scheduling under partially renewable resources and resource consumption during setup operations. Computers \& Industrial Engineering 83:91-99.

Ozdamar L, Alanya E (2001) Uncertainty modelling in software development projects (with case study). Annals of Operations Research 102(1-4): 157-178.

Pan H, Willis RJ, Yeh CH (2001) Resource constrained project scheduling with fuzziness. Advances in fuzzy systems and evolutionary computation, WSEAS Press, pp. 173-179.

Plaza M (2016) Balancing the costs of human resources on an ERP project. Omega 59:171-183.

Pritsker A, Watters L, Wolfe P (1969) Multi-project scheduling with limited resources: A zero-one programming approach. Management Science 16:93-108.

Ranjbar M, Kianfar F, Shadrokh S (2008) Solving the resource availability cost problem in project scheduling by path relinking and genetic algorithm. Applied Mathematics and Computation 196:879-888.

Rezaei F, Najafi AA, Ramezanian R (2020) Mean-conditional value at risk model for the stochastic project scheduling problem. Computers \& Industrial Engineering 142:106356.

Rostami S, Creemers S, Leus R (2018) New strategies for stochastic resource-constrained project scheduling. Journal of Scheduling 21(3): 349-365.

Roghanian E, Alipour M, Rezaei M (2018) An improved fuzzy critical chain approach in order to face uncertainty in project scheduling. International Journal of Construction Management 18(1): 1-13.

Sajadi SM, Azimi P, Ghamginzadeh A, Rahimzadeh A (2017) A new fuzzy multi-objective multi-mode resource constrained project scheduling model. International Journal of Mathematics in Operational Research 11(1): 45-66.

Sallam KM, Chakrabortty RK, Ryan MJ (2021) A reinforcement learning based multi-method approach for stochastic resource constrained project scheduling problems. Expert Systems with Applications 169: 114479.

Shadrokh S, Kianfar F (2007) A genetic algorithm for resource investment project scheduling problem, tardiness permitted with penalty. European Journal of Operational Research 181(1): 86-101.

Sotoudeh-Anvari A (2020) A critical review on theoretical drawbacks and mathematical incorrect assumptions in fuzzy OR methods: Review from 2010 to 2020. Applied Soft Computing 93: 106354.

Subulan K (2020) An interval-stochastic programming based approach for a fully uncertain multi-objective and multi-mode resource investment project scheduling problem with an application to ERP project implementation. Expert Systems with Applications 149: 113189.

Subulan K (2019) An interval programming based approach for fully uncertain resourceconstrained project scheduling problem considering project manager's attitude toward risk. Pamukkale University Journal of Engineering Sciences 25(4): 481-497.

Tadinen H (2005) Human resources management aspects of enterprise resource planning (ERP) systems projects. Master's thesis in Advanced Financial Information Systems, Swedish School of Economics and Business Administration.

Tao S, Dong ZS (2018) Multi-mode resource-constrained project scheduling problem with alternative project structures. Computers \& Industrial Engineering 125: 333-347.

Tseng CC, Ko PW (2016) Measuring schedule uncertainty for a stochastic resource-constrained project using scenario based approach with utility-entropy decision model. Journal of Industrial and Production Engineering, 33(8), 558-567.

Uysal F, Işleyen SK, Çetinkaya C (2018) Resource constrained project scheduling with stochastic resources. Journal of Applied Research on Industrial Engineering 5(1): 39-49.

Wang L, Huang H, Ke H (2015) Chance-Constrained model for RCPSP with uncertain durations. Journal of Uncertainty Analysis and Applications 12(3): 1-10. 
Wang J (2002) A fuzzy project scheduling approach to minimize schedule risk for product development. Fuzzy Sets and Systems 127(2): 99-116.

Wang J (2004) A fuzzy robust scheduling approach for product development projects. European Journal of Operational Research 152: 180-194.

Xiong J, Chen Y, Liu J, Abbass HA (2011) An evolutionary multi-objective scenario-based approach for stochastic resource investment project scheduling. In: IEEE Congress of Evolutionary Computation (CEC), pp. 2767-2774.

Xiong J, Liu J, Chen Y, Abbass HA (2014) A knowledge-based evolutionary multiobjective approach for stochastic extended resource investment project scheduling problems. IEEE Transactions on Evolutionary Computation 18(5):742-763.

$\mathrm{Xu} \mathrm{Z}$, Zhang Z (2012) A fuzzy random resource-constrained scheduling model with multiple projects and its application to a working procedure in a large-scale water conservancy and hydropower construction project. Journal of Scheduling 15: 253-272.

Xu J, Feng C (2014) Multimode resource-constrained multiple project scheduling problem under fuzzy random environment and its application to a large scale hydropower construction project. The Scientific World Journal Article ID 463692: 1-20.

$\mathrm{Xu} \mathrm{J}, \mathrm{Ma} \mathrm{Y}, \mathrm{Xu} \mathrm{Z}$ (2015) A bilevel model for project scheduling in a fuzzy random environment. IEEE Transactions on Systems, Man, and Cybernetics: Systems 45(10): 13221335.

Yamashita DS, Armentano VA, Laguna M (2007) Robust optimization models for project scheduling with resource availability cost. Journal of Scheduling 10(1): 67-76.

Yousefli A (2017) A fuzzy ant colony approach to fully fuzzy resource constrained project scheduling problem. Industrial Engineering \& Management Systems 16(3): 307-315.

Young KD, Feydy T, Schutt A (2017) Constraint programming applied to the multi-skill project scheduling problem. In: J. Christopher Beck, editor, Principles and Practice of Constraint Programming, pages 308-317, Cham. Springer International Publishing.

Zhang Z (2014) A MODM bi-level model with fuzzy random coefficients for resourceconstrained project scheduling problems. In: $7^{\text {th }}$ International Joint Conference on Computational Sciences and Optimization (CSO), Beijing, China, 4-6 July.

Zhang Z, Liu M, Song X (2019) A bi-level fuzzy random model for multi-mode resourceconstrained project scheduling problem of photovoltaic power plant. Journal of Renewable and Sustainable Energy 11(3): 1-15.

Zhang Q, Zhou J, Wang K, Pantelous AA (2018) An effective solution approach to fuzzy programming with application to project scheduling. International Journal of Fuzzy Systems 20: $2383-2398$.

Zhu X, Ruiz R, Li S, Li X (2017) An effective heuristic for project scheduling with resource availability cost. European Journal of Operational Research 257(3): 746-762. 